\title{
Single Commodity Flow Algorithms for Lifts of Graphic and Cographic Matroids
}

\author{
by \\ Leanne Stuive
}

\author{
A thesis \\ presented to the University of Waterloo \\ in fulfillment of the \\ thesis requirement for the degree of \\ Master of Mathematics \\ in \\ Combinatorics and Optimization
}

Waterloo, Ontario, Canada, 2013

(c) Leanne Stuive 2013 
I hereby declare that I am the sole author of this thesis. This is a true copy of the thesis, including any required final revisions, as accepted by my examiners.

I understand that my thesis may be made electronically available to the public. 


\begin{abstract}
Consider a binary matroid $M$ given by its matrix representation. We show that if $M$ is a lift of a graphic or a cographic matroid, then in polynomial time we can either solve the single commodity flow problem for $M$ or find an obstruction for which the Max-Flow MinCut relation does not hold. The key tool is an algorithmic version of Lehman's Theorem for the set covering polyhedron.
\end{abstract}




\section{Acknowledgements}

First and foremost, I would like to thank my supervisor Professor Bertrand Guenin for his excellent guidance. His ongoing assistance and commitment to this research made my thesis a reality.

Secondly, thanks go to my readers Professor Jim Geelen and Professor Levent Tunçel. Additionally, I thank Colton Pauderis for proofreading this document.

Thirdly, I am grateful to my friends, family, and colleagues who provided moral support over the past year and a half - from the people who convinced me to actually show up day one, to those I texted page counts on day 500, and everyone in between.

Lastly, I acknowledge the University of Waterloo and NSERC for funding my graduate studies. 


\section{Table of Contents}

List of Figures $\quad$ ix

1 Introduction $\quad 1$

1.1 Multi-Commodity Flow in Graphs . . . . . . . . . . . . . . . . . . . 1

1.1.1 Flow Definitions . . . . . . . . . . . . . . . . . 1

1.1.2 Bad Instances . . . . . . . . . . . . . . . . . . . 3

1.1.3 Minors ....................... 5

1.1.4 Existing Results . . . . . . . . . . . . . 6

1.2 Multi-Commodity Flow in Binary Matroids . . . . . . . . . . . . . 7

1.2.1 Matroid Theory Background . . . . . . . . . . . 7

1.2.2 Flow Definitions . . . . . . . . . . . . 8

1.2.3 Bad Instances . . . . . . . . . . . . . . . . . . . . . . . . 9

1.2.4 Minors ......................... 13

1.2.5 Existing Results and Conjectures . . . . . . . . . . . . 13

1.2.6 Lifts and Projections of Graphic and Cographic Matroids . . . . . . 14

1.3 Results in This Thesis . . . . . . . . . . . . . . . . . 15

1.3.1 Algorithmic Point of View . . . . . . . . . . . . . . 15

1.3.2 Measuring Efficiency . . . . . . . . . . . . 15

1.3.3 Algorithms Arising from Existential Results . . . . . . . . . . 16

1.4 Overview of Remainder of Thesis . . . . . . . . . . . . . . . . 18 
2 Flow Results and Relationship to Clutters 19

2.1 Flows as a Minimax Relation . . . . . . . . . . . . . . . . . . . . 19

2.1.1 Definitions and Connections . . . . . . . . . . . . . . . 19

2.1.2 Application to Graphic Matroids . . . . . . . . . . . . . . . . 21

2.1.3 Application to Cographic Matroids . . . . . . . . . . . . . . . 21

2.2 Multi-Commodity to Single Commodity Flow . . . . . . . . . . . . . 22

2.2 .1 Equivalence . . . . . . . . . . . . . . . . . . . . . . . 22

2.2.2 Reformulating Single Commodity Flow Using e-Paths . . . . . . 24

2.2.3 Single Commodity Flow for Graphic Matroids . . . . . . . . . 26

2.2.4 Single Commodity Flow for Cographic Matroids . . . . . . . . . 26

2.3 Lifts and Projections of Graphic and Cographic Matroids _ . . . . . . 26

2.3.1 Cycle and Cocycle Spaces for Binary Matroids . . . . . . . . . . 26

2.3 .2 Even Cycle Matroids . . . . . . . . . . . . . . . . . . . 27

2.3 .3 Even Cut Matroids . . . . . . . . . . . . . . . . . . . . . 28

2.4 Single Commodity Flow Results _ . . . . . . . . . . . . . . . 28

2.4 .1 Even Cycle Matroids . . . . . . . . . . . . . . . . . . . . . . . . . . . 29

2.4.2 Even Cycle Matroids: $e$ a loop . . . . . . . . . . . . . . . . 29

2.4 .3 Even Cut Matroids . . . . . . . . . . . . . . . . . . 30

2.4.4 Duals of Even Cycle Matroids . . . . . . . . . . . . . . . 30

2.4.5 Duals of Even Cut Matroids _. . . . . . . . . . . . . . 31

2.4.6 A Note on the Restriction to Binary Matroids . . . . . . . . . 32

2.5 Relating Flows to Clutters _. . . . . . . . . . . . . . . . 32

2.5 .1 Definitions . . . . . . . . . . . . . . . . . . . . . 32

2.5 .2 The MFMC Properties . . . . . . . . . . . . . . . . 33

2.5.3 Flowing and Cycling Conjectures for Clutters . . . . . . . . 34

2.5.4 Flowing and Cycling Results for Clutters . . . . . . . . . . . 35 
3 A Constructive Version of Lehman's Theorem 36

3.1 Preliminaries . . . . . . . . . . . . . . . . . . . . 36

3.1.1 Set Covering Polyhedra, Idealness, and Lehman's Theorem . . . . . 36

3.1 .2 Statement of Constructive Version . . . . . . . . . . . . . . 37

3.2 Proof Outline . . . . . . . . . . . . . . . . . . . . . . . . . . 38

3.2 .1 Key Definitions and Lemmas _ . . . . . . . . . . . . . . 38

3.2 .2 Algorithm . . . . . . . . . . . . . . . . . . . . . 40

3.3 Technical Sections of Proof $\ldots \ldots \ldots 4$. . . . . . . . . . 41

3.3 .1 Polyhedral Proofs . . . . . . . . . . . . . . . . . . . . . . 41

3.3 .2 Proofs using Property $(\mathrm{P}) \ldots \ldots \ldots \ldots$

3.3 .3 Proof of Lemma $3.2 .6 \ldots \ldots \ldots \ldots$

3.4 Exploring Lehman's Theorem _ . . . . . . . . . . . . . . . . . 47

3.4.1 Interpreting Lehman's Theorem: $\mathcal{C}_{2,3}$ and $\mathcal{O}_{K_{5}} \ldots \ldots \ldots . \ldots 47$

3.4 .2 Useful Corollaries . . . . . . . . . . . . . . . . . . . . . . . . . . 48

4 Applications to Odd-st-Walks and $s t$-T-Cuts $\quad 51$

4.1 Chapter Overview . . . . . . . . . . . . . . . . . . . . . . . . . . 51

4.2 Minors in Signed Graphs and Grafts _ . . . . . . . . . . . . . . 52

4.3 Proof Outline . . . . . . . . . . . . . . . . . . . . . 54

4.3.1 Proof Outline: Theorem 4.1.1 _ . . . . . . . . . . . . . 54

4.3 .2 Proof Outline: Theorem $4.1 .2 \ldots \ldots \ldots$

4.4 Solving the Separation Problems _ . . . . . . . . . . . . . 57

4.4.1 The Separation Problem for Odd-st-Walks . . . . . . . . . . 57

4.4.2 The Separation Problem for st-T-Cuts . . . . . . . . . . . . . 59

4.5 Proof of Lemma $4.3 .3 \ldots \ldots$. . . . . . . . . . . . . . . . 61

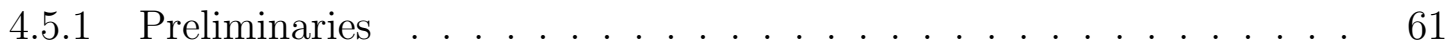

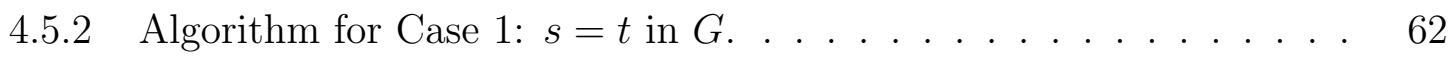

4.5.3 Algorithm for Case 2: $s \neq t$ in G. . . . . . . . . . 63 
4.5.4 Proof for Case 1: $s=t$ in $G \ldots \ldots \ldots \ldots \ldots$

4.5.5 Proof for Case $2: s \neq t$ in $G \ldots \ldots \ldots \ldots \ldots$

4.6 Proof of Lemma $4.3 .5 \ldots \ldots \ldots$. . . . . . . . . . . . . 67

4.6 .1 Preliminaries . . . . . . . . . . . . . . . . . . . . 68

4.6.2 Algorithm for Case 1: $\ell \geq 4 \ldots \ldots . \ldots . \ldots 9$

4.6.3 Proof of Correctness for Case $1: \ell \geq 4 \ldots \ldots \ldots$

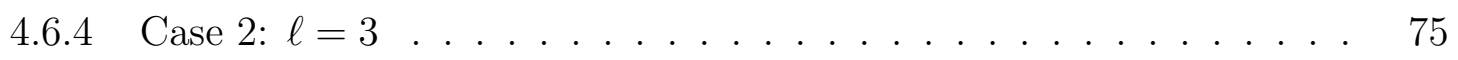

4.7 Remarks . . . . . . . . . . . . . . . . . 77

5 Future Work, Related Problems and Conclusions $\quad 78$

5.1 Future Work: Eulerian Multi-Commodity Flow Algorithms . . . . . . . . 78

5.1.1 Transversal Property, Underflows and Overflows . . . . . . . . 79

5.1.2 Finite Time Algorithm Arising from Theorem 1.1.4 . . . . . . . 80

5.1 .3 Capacity Scaling . . . . . . . . . . . . . . . . 81

5.2 Other Related Problems _. . . . . . . . . . . . . . . . . . 83

5.2 .1 Cuts with Parity Conditions _ . . . . . . . . . . 83

5.2 .2 Minimally Imperfect Graphs . . . . . . . . . . . . . . . . . 84

5.3 Conclusions . . . . . . . . . . . . . . . . . . . . . . . 84

$\begin{array}{lr}\text { References } & 85\end{array}$ 


\section{List of Figures}

1.1 Multi-Commodity Flow Instance . . . . . . . . . . . . . . . . 2

1.2 Signed Graph Odd $-K_{4} \ldots \ldots \ldots$. . . . . . . . . . . . 4

1.3 Signed Graph Odd $-K_{5} \ldots \ldots \ldots$. . . . . . . . . . . . . 5

$1.4 \mathrm{Odd}-K_{4} \ldots \ldots \ldots \ldots \ldots$

$1.5 \mathrm{Odd}-K_{5} \ldots \ldots \ldots \ldots \ldots$

1.6 Complements of Cuts of Odd $K_{5} \ldots \ldots \ldots \ldots$

1.7 Lines of Fano Matroid . . . . . . . . . . . . . . . . . . . . . 11

1.8 Postman Sets of Petersen Graph . . . . . . . . . . . . . . . . . . . 12

2.1 Cographic Odd $K_{4} \ldots \ldots \ldots \ldots \ldots$

$2.2 \mathrm{AG}(3,2) \ldots \ldots \ldots \ldots \ldots \ldots \ldots$

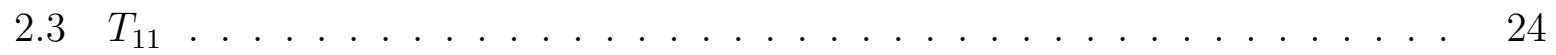

4.1 Uncrossing Illustration . . . . . . . . . . . . . . . . . . . 60

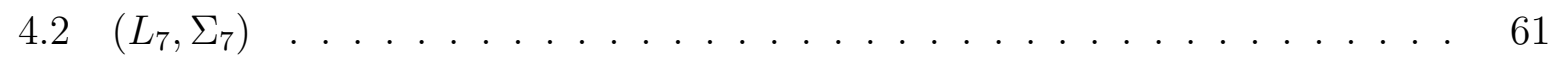

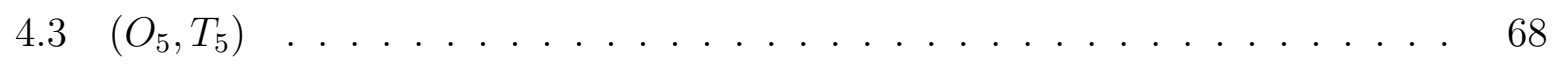

$4.4\left(L_{1}, T_{1}\right),\left(L_{2}, T_{2}\right) \ldots \ldots \ldots \ldots$ 


\section{Chapter 1}

\section{Introduction}

\subsection{Multi-Commodity Flow in Graphs}

\subsubsection{Flow Definitions}

A graph $G=(V, E)$, weight function $w \in \mathbb{Z}_{+}^{E}$, and set of demand edges $\Sigma \subseteq E$ determine a multi-commodity flow instance. Each $d \in \Sigma$ is a demand edge with demand $w_{d}$ while each $e \in E-\Sigma^{1}$ is a capacity edge with capacity $w_{e}$. Given a graph $G=(V, E), C \subseteq E$ is a circuit of $G$ if $G[C]$ is connected and 2-regular. Let $\mathcal{C}_{1}$ be the set of circuits of $G$ intersecting $\Sigma$ in exactly one demand edge. Call $y \in \mathbb{R}_{+}^{\mathcal{C}_{1}}$ a (fractional) $w$-flow if it satisfies

(1) demand constraints: for each $d \in \Sigma: \quad \sum\left(y_{C}: d \in C \in \mathcal{C}_{1}\right)=w_{d}$

(2) capacity constraints: for each $e \in E \backslash \Sigma: \quad \sum\left(y_{C}: e \in C \in \mathcal{C}_{1}\right) \leq w_{e}$.

A cut of $G$ is a set of edges $\delta(S) \subseteq E$ for $S \subseteq V$ where $\delta(S)$ is defined as the set of edges with exactly one endpoint in $S$. That is, $\delta(S):=\{e=x y: x \in S, y \notin S\}$. In the future, $\delta(v)$ will denote the set $\delta(\{v\})$. The cut condition is a necessary condition for the existence of a fractional $w$-flow. It states that the demand across any cut should not exceed the capacity across the cut. Letting $w(F):=\sum\left(w_{e}: e \in F\right)$ for $F \subseteq E$, the cut condition states that for all $S \subseteq V, w(\delta(S)-\Sigma) \geq w(\delta(S) \cap \Sigma)$.

A signed graph is a pair $(G, \Sigma)$ where $G$ is a graph and $\Sigma \subseteq E(G)$. We say $B \subseteq E$ is odd if $|B \cap \Sigma|$ is odd. We say $B \subseteq E$ is even if $|B \cap \Sigma|$ is even. In particular, edges in $\Sigma$

\footnotetext{
${ }^{1}$ Here '-' indicates set difference and ' $A-a$ ' denotes $A-\{a\}$
} 
are odd. If $C$ is a circuit of $G$ and $|C \cap \Sigma|$ is odd, we call $C$ an odd circuit of $(G, \Sigma)$. Given $(G, \Sigma)$, updating $\Sigma$ to $\Sigma^{\prime}=\Sigma \triangle \delta(S)$ for $S \subseteq V$ does not change the set of odd circuits in $(G, \Sigma) .{ }^{2}$ Any $\Sigma^{\prime}$ that can be obtained in this way is called a signature of $(G, \Sigma)$. The cut condition can be reformulated using signatures as follows. For a proof, see Remark 1.2.1.

Remark 1.1.1. The cut condition holds for $(G, \Sigma)$ if and only if $\Sigma$ is a minimum weight signature of $(G, \Sigma)$.

If $w \in \mathbb{Z}_{+}^{E}$ satisfies the cut condition, then triple $(G, \Sigma, w)$ defines two multi-commodity flow problems. The fractional multi-commodity flow problem asks if there exists a fractional $w$-flow $y \in \mathbb{R}_{+}^{\mathcal{C}_{1}}$. The integer multi-commodity flow problem asks if there exists an integer $w$-flow $y \in \mathbb{Z}_{+}^{\mathcal{C}_{1}}$.

Finding an integer $w$-flow is equivalent to assigning to each $C \in \mathcal{C}$ an integer multiplicity $y_{C}$ subject to two restrictions: (1) the total multiplicities of circuits through $e \notin \Sigma$ must not exceed $w_{e}$, and, (2) the total multiplicity of circuits through $d \in \Sigma$ must equal $w_{d}$. If $w_{e}=1$ for all $e \in E$, the instance is said to have unit weights and a $w$-flow is called an $\mathbb{1}$-flow. Finding a $\mathbb{1}$-flow corresponds to choosing for each $d \in \Sigma$ a circuit whose intersection with $\Sigma$ is exactly $\{d\}$, with the restriction that the circuits chosen are pairwise disjoint. This viewpoint foreshadows the connection to packing discussed in Section 2.4.2. Figure 1.1 demonstrates some of the definitions introduced thus far.

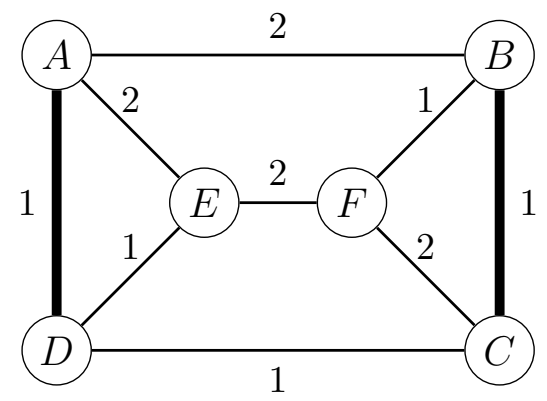

Figure 1.1: Multi-Commodity Flow Instance: Demand edges are the thick edges $(A D$ and $B C)$. Edge $e$ is labelled by weight $w_{e}$. If $C_{1}=\{A E, D E, A D\}, C_{2}=\{B C, C F, B F\}$ and $C_{3}=\{B C, C F, E F, A E, A B\}$, then $C_{1} \in \mathcal{C}_{A D}$ and $C_{2}, C_{3} \in \mathcal{C}_{B C}$. The assignment $y_{C_{1}}=1$, $y_{C_{2}}=y_{C_{3}}=1 / 2$ specifies a fractional $w$-flow. The assignment $y_{C_{1}}=1, y_{C_{2}}=1$ specifies an integer $w$-flow. Note that $C_{1}$ and $C_{2}$ are disjoint circuits of $G$.

\footnotetext{
${ }^{2}$ Here $\triangle$ denotes symmetric difference; $A \triangle B=(A \backslash B) \cup(B \backslash A)$.
} 
The role of $(G, \Sigma)$ in the existence of multi-commodity flows for all weight functions is of interest. A weight function $w \in \mathbb{Z}_{+}^{E}$ is called Eulerian if $w(\delta(v))$ is even for all $v \in V$. The role of $(G, \Sigma)$ in the existence of integer flows for all Eulerian weight functions is also of interest. We look for sufficient conditions; what guarantees regarding the structure of $(G, \Sigma)$ imply a flow exists for all weight functions $w \in \mathbb{Z}_{+}^{E}$ ? Although this may seem less general, it ensures our results speak only to the structure of $(G, \Sigma)$. Given $(G, \Sigma)$ and $w$ satisfying the cut condition, consider the following problems.

1. Fractional Flow Problem: Does there exist a fractional $w$-flow?

2. Integer Flow Problem: Does there exist an integer $w$-flow?

3. Eulerian Integer Flow Problem: If $w$ is Eulerian, does there exist an integer $w$-flow?

Clearly, if there is an integer $w$-flow for every $w \in \mathbb{Z}_{+}^{E}$ satisfying the cut condition, necessarily there is a fractional $w$-flow for all such $w$. In fact, if there exists an integer $w$-flow for every Eulerian $w \in \mathbb{Z}_{+}^{E}$ satisfying the cut condition, there is a fractional $w$-flow for all $w$ satisfying the cut condition. To see this, note that given $w \in \mathbb{Z}_{+}^{E}, 2 w$ is necessarily an Eulerian weight function and also satisfies the cut condition. By assumption, there exists a $2 w$-flow $y ; \frac{1}{2} y$ is a fractional $w$-flow.

It may not be immediately obviously that there exist "bad instances". A bad instance for the fractional flow problem is a triple $(G, \Sigma, w)$ where $w$ satisfies the cut condition but $(G, \Sigma)$ does not admit a fractional $w$-flow. Likewise, a bad instance for the integer flow problem is a triple $(G, \Sigma, w)$ where $w$ satisfies the cut condition but $(G, \Sigma)$ does not admit an integer $w$-flow. The two important signed graphs introduced next demonstrate that such instances exist.

\subsubsection{Bad Instances}

Odd- $K_{n}$

Given signed graph $(G, \Sigma)$, the set of signed graphs $\left(G, \Sigma^{\prime}\right)$ such that $\Sigma^{\prime}=\Sigma \triangle \delta(S)$ for $S \subseteq V$ form an equivalence class. Note that $(G, \Sigma)$ and $\left(G, \Sigma^{\prime}\right)$ are equivalent if and only if $\Sigma^{\prime}$ is a signature of $(G, \Sigma)$. We may refer to $\left(G, \Sigma^{\prime}\right)$ as a resigning of $(G, \Sigma)$. We let odd- $K_{n}$ denote the equivalence class containing $\left(K_{n}, E\left(K_{n}\right)\right)$ where $K_{n}$ denotes the complete graph on $n$ vertices. 


\section{Odd- $K_{4}$}

Consider a flow problem on the graph $K_{4}$. The two demand edges $\left(\Sigma_{4}\right)$ are a pair of nonadjacent edges in $K_{4}$. The weight function is unit: $w_{e}=1$ for all $e \in E$. This instance is illustrated in Figure 1.2. Resigning on $\delta(\{A, B\})$ shows this signed graph is in the equivalence class odd- $K_{4}$.

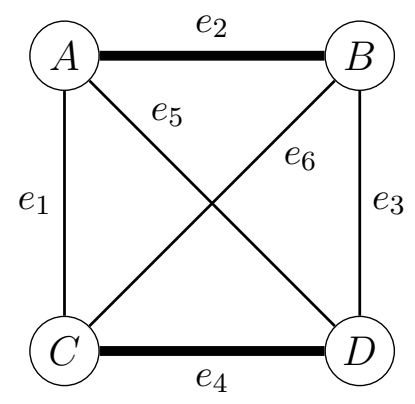

Figure 1.2: Graph is $K_{4}$. Each edge is labelled by its name. Thick edges are demand edges.

The circuits intersecting $\Sigma_{4}$ exactly in the edge $e_{2}$ are $\mathcal{C}_{e_{2}}=\left\{\left\{e_{2}, e_{3}, e_{5}\right\},\left\{e_{1}, e_{2}, e_{6}\right\}\right\}$. The circuits intersecting $\Sigma_{4}$ exactly in the edge $e_{4}$ are $\mathcal{C}_{e_{4}}=\left\{\left\{e_{1}, e_{4}, e_{5}\right\},\left\{e_{3}, e_{4}, e_{6}\right\}\right\}$. Assigning $1 / 2$ to each $C \in \mathcal{C}_{1}$, we obtain a fractional flow for the unit weight function. That is, $y_{\left\{e_{2}, e_{3}, e_{5}\right\}}=y_{\left\{e_{1}, e_{2}, e_{6}\right\}}=y_{\left\{e_{1}, e_{4}, e_{5}\right\}}=y_{\left\{e_{3}, e_{4}, e_{5}\right\}}=1 / 2$ is a 1 -flow. Since the cut condition is necessary for the existence of a fractional $w$-flow, the fractional $\mathbb{1}$-flow $y$ demonstrates that the cut condition holds.

This instance, however, does not have an integer flow. Finding an integer flow is equivalent to finding $C_{2} \in \mathcal{C}_{e_{2}}$ and $C_{4} \in \mathcal{C}_{e_{4}}$ such that $C_{2} \cap C_{4}=\emptyset$. It is straightforward to verify that no such $C_{2}$ and $C_{4}$ exist.

\section{Odd- $K_{5}$}

Consider a flow problem on the graph $K_{5}$. There are four demand edges: any triangle plus the unique remaining edge not adjacent to a triangle edge. Again consider the unit weight function. Note that $w(\delta(v))=|\delta(v)|=4$ for all $v \in V$; the weight function is Eulerian. This instance is illustrated in Figure 1.3. Resigning on $\delta(\{A, B, C\})$ shows this signed graph is in the equivalence class odd $-K_{5}$. 


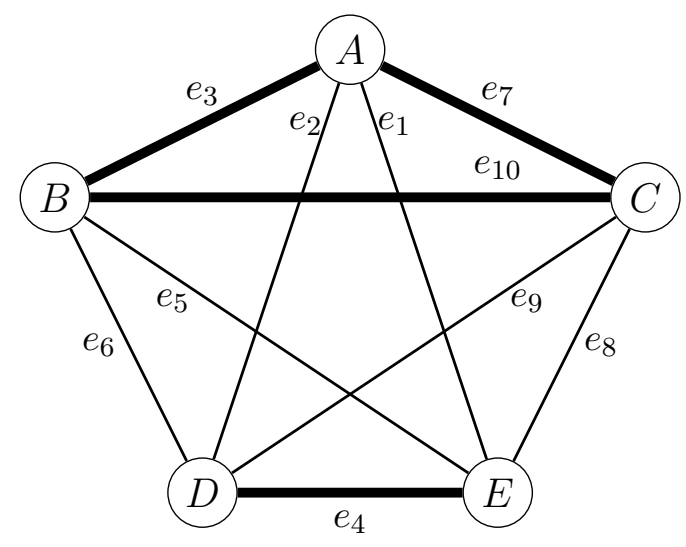

Figure 1.3: Graph is $K_{5}$. Each edge is labelled by its name. Thick edges are demand edges.

It is straightforward (but tedious) to verify that the cut condition holds for this instance. However, there does not exist a fractional flow. The total demand is $w\left(\Sigma_{5}\right)=4$ and the total capacity is $w\left(E \backslash \Sigma_{5}\right)=6$. Since each circuit using exactly one demand edge has length exactly three, it requires $2 \epsilon$ units of capacity to satisfy $\epsilon$ units of demand and so 8 units of capacity are required to route the 4 units of demand. Only 6 units of capacity is available and therefore no fractional flow exists. Obviously, if there is no fractional flow then there is no integer flow. In sum, this is an instance with an Eulerian weight function, but no fractional or integer flow.

\subsubsection{Minors}

We return to the question of interest: what conditions can be imposed on $(G, \Sigma)$ to guarantee the existence of integer or fractional flows for all (or all Eulerian) weight functions? Having established this is a reasonable question, we first require a mechanism for ascribing structure to $(G, \Sigma)$.

Graph minors are frequently used to classify the structure of (unsigned) graph $G$. Given $G=(V, E)$, there are two minor operations: deletion and contraction. Deleting edge $e \in E$ is done by removing $e$; the resulting graph is denoted by $G \backslash e$. In other words, we define $G \backslash e$ as $(V, E-e)$. Contracting edge $e \in E$ is done by deleting $e$ and identifying its endpoints; the resulting graph is denoted by $G / e$. For $e=x y$, we define $G / e$ as $\left(V \cup\{z\}-\{x, y\}, E^{\prime}\right)$ with $E^{\prime}$ defined as follows: if $f \in E$ and neither endpoint of $f$ is $x, y$, then $f \in E^{\prime}$; if $f \in E$ 
and $f=s x$ or $f=x y$ then $s z \in E^{\prime}$. A minor of $G$ is any graph obtained from $G$ by a sequence of contractions and deletions.

Graph minor operations can be thought to act on the circuits of the graph. Deleting edge $e$ results in removing from $G$ all the circuits that used edge $e$ and otherwise preserving the set of circuits. Contracting edge $e$ results in removing the edge $e$ from all the circuits in $G$ using edge $e$ and otherwise preserving the set of circuits. It is straightforward to verify that deletions and contractions can be performed in any order. Namely, if $G^{\prime}$ is obtained from $G$ by deleting edges in $I$ and contracting edges in $J$ in some order, then we can unambiguously denote $G^{\prime}$ by $G / I \backslash J$. One way to classify a graph is according to whether it contains a certain fixed minor; $G$ contains $G^{\prime}$ as a minor if and only if there exists $I, J \subseteq E(G)$ such that $G^{\prime}=G / I \backslash J$.

Although graph minors characterize the structure of graph $G$, we are interested in the structure of the signed graph $(G, \Sigma)$. The idea of minors in graphs can be extended to minors in signed graphs. Recall that given $(G, \Sigma)$, updating $\Sigma$ to $\Sigma^{\prime}=\Sigma \triangle \delta(S)$ for $S \subseteq V$ does not change the set of odd circuits in $(G, \Sigma)$. We call this operation resigning. Given $(G, \Sigma)$ and $e \in E$, we define $(G, \Sigma) \backslash e$ as $(G \backslash e, \Sigma-e)$ and call this operation deletion. Given $(G, \Sigma)$ and $e \in E$ that is not an odd loop (edge with same endpoints), suppose we resign to obtain $\Sigma^{\prime}$ such that $e \notin \Sigma^{\prime}$. We define $(G, \Sigma) / e$ as $\left(G / e, \Sigma^{\prime}\right)$ and call this operation contraction. A minor of $(G, \Sigma)$ is any signed graph $\left(G^{\prime}, \Sigma^{\prime}\right)$ obtained from $(G, \Sigma)$ by a sequence of contractions and deletions. Deleting $e$ in $(G, \Sigma)$ removes all the circuits using $e$. Contracting $e \in(G, \Sigma)$ shortens all the circuits using $e$ while maintaining their parity.

Since minor operations can be applied in any order, we denote by $(G, \Sigma) / I \backslash J$ the signed graph obtained by contracting all edges in $I \subseteq E$ and deleting all edges in $J \subseteq E$ (and resigning in whatever way avoids contracting signature edges). Necessarily $I$ does not contain an odd circuit. One way to classify a signed graph is according to whether it contains a certain fixed minor; $(G, \Sigma)$ contains $\left(G^{\prime}, \Sigma^{\prime}\right)$ as a minor if and only if there exists $I, J \subseteq E(G)$ such that $\left(G^{\prime}, \Sigma^{\prime \prime}\right)=(G, \Sigma) / I \backslash J$ and $\Sigma^{\prime}=\Sigma^{\prime \prime} \triangle \delta_{G^{\prime}}(U)$ for $U \subseteq V\left(G^{\prime}\right)$. Note that this definition does not account explicitly for the resigning operation. If $\left(G^{\prime}, \Sigma^{\prime}\right)$ is a minor of $(G, \Sigma)$ then so is any resigning of $\left(G^{\prime}, \Sigma^{\prime}\right)$. In other words, writing " $\left(G^{\prime}, \Sigma^{\prime}\right)$ is a minor of $(G, \Sigma)$ " means that every resigning of $\left(G^{\prime}, \Sigma^{\prime}\right)$ is a minor of every resigning of $(G, \Sigma)$.

\subsubsection{Existing Results}

Having defined minors, we return to sufficient conditions for the existence of flows. One way to give such conditions is by providing a list of minors with the property that whenever 
$(G, \Sigma)$ contains no minor on the list, $(G, \Sigma)$ has a flow for every $w \in \mathbb{Z}_{+}^{E}$. Such lists are known for the flow problems of interest.

Theorem 1.1.2 (Seymour [29]). Suppose $(G, \Sigma)$ has no odd- $K_{4}$ minor. Then for every $w \in \mathbb{Z}_{+}^{E}$ satisfying the cut condition, $(G, \Sigma)$ has an integer $w$-flow.

Theorem 1.1.3 (Guenin [18]). Suppose $(G, \Sigma)$ has no odd- $K_{5}$ minor. Then for every weight function $w \in \mathbb{Z}_{+}^{E}$ satisfying the cut condition, $(G, \Sigma)$ has a fractional $w$-flow.

Theorem 1.1.4 (Geelen, Guenin [13]). Suppose $(G, \Sigma)$ has no odd- $K_{5}$ minor. Then for every Eulerian weight function $w \in \mathbb{Z}_{+}^{E}$ satisfying the cut condition, $(G, \Sigma)$ has an integer $w$-flow.

\subsection{Multi-Commodity Flow in Binary Matroids}

\subsubsection{Matroid Theory Background}

A matroid $M$ is a pair $(E, \mathcal{I})$ where $E:=E(M)$ is a finite set called the ground set, and $\mathcal{I} \subseteq 2^{E}$ is a set of subsets of $E$ satisfying the following properties:

(I1) $\emptyset \in \mathcal{I}$,

(I2) if $J \in \mathcal{I}$ and $I \subseteq J$, then $I \in \mathcal{I}$, and

(I3) if $X \subseteq E$ and $I_{1}$ and $I_{2}$ are maximal members of $\{I: I \in \mathcal{I}$ and $I \subseteq X\}$, then $\left|I_{1}\right|=\left|I_{2}\right|$.

Set $I \in \mathcal{I}$ is called independent; set $I \notin \mathcal{I}$ is called dependent. We call a maximally independent subset of $E$ a basis of $M$. Property (I3) shows that all bases have the same size, called the rank of the matroid. Let $C \subseteq E ; C$ is a circuit of $M$ if it is an inclusion-wise minimally dependent set. A matroid is uniquely determined by its set of circuits.

A matroid $M$ with ground set $E=\left\{e_{1}, \ldots e_{n}\right\}$ is said to be representable over field $\mathbb{F}$ if there exists a matrix $A \in \mathbb{F}^{m \times n}$ with columns indexed by $\left\{e_{1} \ldots e_{n}\right\}$ such that the independent sets of $M$ coincide exactly with the column sets of $A$ that are linearly independent over $\mathbb{F}$. Matrix $A$ is called a matrix representation for $M$. If $M$ is representable over $G F(2)$, then $M$ is called binary. In this paper, we are primarily concerned with binary matroids. In a binary matroid, a cycle is any finite symmetric difference of circuits.

The dual of matroid $M$, denoted $M^{*}$, is the matroid with ground set $E$ such that $S \subseteq E$ is independent if and only if there exists a basis $B$ of $M$ such that $S \cap B=\emptyset$. The circuits of $M^{*}$ are called the cocircuits of $M$. In binary matroids, each cocircuit intersects each 
circuit with even parity. A cocyle of a binary matroid is any finite symmetric difference of cocircuits.

A matroid $M$ is graphic if there exists a graph $G$ such that the circuits of $M$ correspond exactly to the circuits of $G$. Given graph $G$, let $M(G)$ denote the affiliated graphic matroid. One possible matrix representation for $M(G)$ is the adjacency matrix of $G$. A matroid $M$ is cographic if there exists a graph $G$ such that circuits of $M$ correspond exactly to the minimal cuts of $G$. Given graph $G$, let $M^{*}(G)$ denote the affiliated cographic matroid. As this notation suggests, $M^{*}(G)$ is the dual of $M(G)$. All graphic and cographic matroids are binary and thus can be represented by matrices over $G F(2)$. However, these matroids may also be represented by the underlying graph $G=(V, E)$.

\subsubsection{Flow Definitions}

As matroids are a generalization of graphs, flows in matroids are a generalization of flows in graphs. Throughout this section, the term matroid will refer to a binary matroid. Many of these definitions extend directly to general matroids; Section 2.4.6 justifies the restriction to binary matroids.

A matroid $M$ on ground set $E$, weight function $w \in \mathbb{Z}_{+}^{E}$ and set of demand edges $\Sigma \subseteq E$ determine a flow instance. Let $\mathcal{C}_{1}$ be the set of circuits in $M$ that intersect $\Sigma$ in exactly one element. Call $y \in \mathbb{R}_{+}^{\mathcal{C}_{1}}$ a (fractional) $w$-flow if it satisfies

(1) demand constraints: for each $d \in \Sigma$ :

$$
\begin{aligned}
& \sum\left(y_{C}: d \in C \in \mathcal{C}_{1}\right)=w_{d}, \\
& \sum\left(y_{C}: e \in C \in \mathcal{C}_{1}\right) \leq w_{e} .
\end{aligned}
$$

(2) capacity constraints: for each $e \in E \backslash \Sigma$ :

The cut condition is again necessary for the existence of a fractional $w$-flow. For graphs, the cut condition states that $w(D \cap \Sigma) \leq w(D \backslash \Sigma)$ for all cuts $D=\delta(S)$. Minimal cuts in graphs are the cocircuits of a graphic matroid. The natural extension of the cut condition to arbitrary matroid $M$ states that $w(D \cap \Sigma) \leq w(D \backslash \Sigma)$ for all cocircuits $D$ of $M$.

A signed matroid is a pair $(M, \Sigma)$ where $M$ is a matroid and $\Sigma \subseteq E(M)$. We say $B \subseteq E$ is odd if $|B \cap \Sigma|$ is odd. We say $B \subseteq E$ is even if $|B \cap \Sigma|$ is even. In particular, edges in $\Sigma$ are odd. If $C$ is a circuit of $M$ and $|C \cap \Sigma|$ is odd, we call $C$ an odd circuit of $(M, \Sigma)$. Given $(M, \Sigma)$, updating $\Sigma$ to $\Sigma^{\prime}=\Sigma \triangle D$ for cocycle $D$ of $M$ does not change the set of odd circuits. Any $\Sigma^{\prime}$ that can be obtained in this way is called a signature of $(M, \Sigma)$. We say $(M, \Sigma)$ and $\left(M^{\prime}, \Sigma^{\prime}\right)$ are equivalent and call $\left(M^{\prime}, \Sigma^{\prime}\right)$ a resigning of $(M, \Sigma)$ if $M=M^{\prime}$ and $\Sigma^{\prime}=\Sigma \triangle D$ for some cocycle $D$. The cut condition can be reformulated using signatures as follows. 
Remark 1.2.1. The cut condition holds for $(M, \Sigma)$ if and only if $\Sigma$ is a minimum weight signature of $(M, \Sigma)$.

Proof. The cut condition holds if and only if $w(D \cap \Sigma) \leq w(D \backslash \Sigma)$ for all cocircuits $D$ of $M$. If the cut condition holds, let $\Sigma^{\prime}=\Sigma \triangle D$ be an arbitrary signature of $(M, \Sigma)$. Then

$$
w\left(\Sigma^{\prime}\right)=w(\Sigma \triangle D)=w(\Sigma \backslash D)+w(D \backslash \Sigma) \geq w(\Sigma \backslash D)+w(D \cap \Sigma)=w(\Sigma)
$$

where the inequality $(\geq)$ holds as a result of the cut condition. If the cut condition does not hold, then $w(D \cap \Sigma)>w(D \backslash \Sigma)$ for some cocircuit $D$ of $M$ and so the above inequality for $\Sigma^{\prime}=\Sigma \triangle D$ yields $w\left(\Sigma^{\prime}\right)<w(\Sigma)$. It follows that the cut condition holds for $(M, \Sigma)$ if and only if $\Sigma$ is a minimum weight signature of $(M, \Sigma)$.

If $w \in \mathbb{Z}_{+}^{E}$ satisfies the cut condition, then triple $(M, \Sigma, w)$ defines two multi-commodity flow problems. The fractional multi-commodity flow problem asks if there exists a fractional $w$-flow $y \in \mathbb{R}_{+}^{\mathcal{C}_{1}}$. The integer multi-commodity flow problem asks if there exists an integer $w$-flow $y \in \mathbb{Z}_{+}^{\mathcal{C}_{1}}$.

The questions of interest about flows in graphs can be extended to questions about flows in matroids. In particular, we are concerned with signed matroids $(M, \Sigma)$ for which flows exist for all weight functions $w \in \mathbb{Z}_{+}^{E}$. For graphs, the Eulerian condition states that $w(\delta(v))$ is even for all $v \in V$. Since minimal cuts in graphs are the cocircuits of a graphic matroid, the natural extension to matroids defines weight function $w \in \mathbb{Z}_{+}^{E(M)}$ to be Eulerian if $w(D)$ is even for all cocircuits $D$ of $M$. Given matroid $M, \Sigma \subseteq E$, and $w \in \mathbb{Z}_{+}^{E}$ satisfying the cut condition, the following flow problems arise.

1. Fractional Flow Problem: Does there exist a fractional $w$-flow?

2. Integer Flow Problem: Does there exist an integer $w$-flow?

3. Eulerian Integer Flow Problem: If $w$ is Eulerian, does there exist an integer $w$-flow?

Again we are interested in "bad instances"; $(M, \Sigma, w)$ is a bad instance if $w$ satisfies the cut condition, but there exists no integer flow or no fractional flow.

\subsubsection{Bad Instances}

\section{Odd- $K_{4}$}

The matroid $M\left(K_{4}\right)$ is the graphic matroid corresponding to $K_{4}$. We will overload notation and refer to the equivalence class containing signed matroid $\left(M\left(K_{4}\right), E\left(K_{4}\right)\right)$ as odd- $K_{4}$. 
Considering signature $\Sigma_{4}=\left\{e_{2}, e_{4}\right\}$, the odd circuits of $\left(M\left(K_{4}\right), \Sigma_{4}\right)$ are exactly the odd circuits of the complete graph $K_{4}$. As per the discussion in Section 1.1.2, the unit weight function satisfies the cut condition, but the signed matroid $\left(M\left(K_{4}\right), \Sigma_{4}\right)$ does not have an integer $\mathbb{1}$-flow.

$$
\left[\begin{array}{llllll}
1 & 1 & 0 & 0 & 1 & 0 \\
0 & 1 & 1 & 0 & 0 & 1 \\
1 & 0 & 0 & 1 & 0 & 1 \\
0 & 0 & 1 & 1 & 1 & 0
\end{array}\right], \Sigma_{4}=\left\{e_{2}, e_{4}\right\}
$$

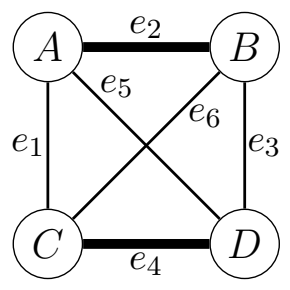

Figure 1.4: Odd- $K_{4}$ : Matrix and graphic representations for $\left(K_{4}, \Sigma_{4}\right)$. Columns of matrix are indexed in order by $\left\{e_{1}, e_{2}, e_{3}, e_{4}, e_{5}, e_{6}\right\}$

\section{Odd- $K_{5}$}

The matroid $M\left(K_{5}\right)$ is the graphic matroid corresponding to $K_{5}$. We will refer to the equivalence class containing signed matroid $\left(M\left(K_{5}\right), E\left(K_{5}\right)\right)$ as odd- $K_{5}$. Considering signature $\Sigma_{5}=\left\{e_{3}, e_{4}, e_{7}, e_{10}\right\}$, the odd circuits of $\left(M\left(K_{5}\right), \Sigma_{5}\right)$ are exactly the odd circuits of the complete graph $K_{5}$. As per the discussion in Section 1.1.2, the unit weight function is Eulerian and satisfies the cut condition, but the signed matroid $\left(M\left(K_{5}\right), \Sigma_{5}\right)$ does not have a fractional $\mathbb{1}$-flow.

$$
\begin{aligned}
& {\left[\begin{array}{llllllllll}
1 & 1 & 1 & 0 & 0 & 0 & 1 & 0 & 0 & 0 \\
0 & 0 & 1 & 0 & 1 & 1 & 0 & 0 & 0 & 1 \\
0 & 0 & 0 & 0 & 0 & 0 & 1 & 1 & 1 & 1 \\
0 & 1 & 0 & 1 & 0 & 1 & 0 & 0 & 1 & 0 \\
1 & 0 & 0 & 1 & 1 & 0 & 0 & 1 & 0 & 0
\end{array}\right],} \\
& \Sigma_{5}=\left\{e_{3}, e_{4}, e_{7}, e_{10}\right\}
\end{aligned}
$$

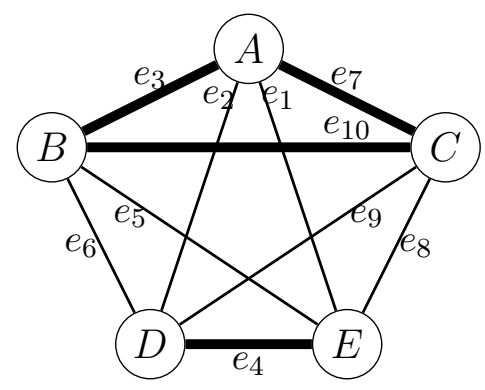

Figure 1.5: Odd- $K_{5}$ : Matrix and graphic representations for $\left(K_{5}, \Sigma_{5}\right)$.

\section{Complements of Cuts of Odd- $K_{5}$}

Consider the binary matroid $T_{10}$ with matrix representation given by Figure 1.6. Let $\Sigma_{10}=\left\{e_{1}, e_{7}, e_{8}\right\}$. The odd circuits of $\left(T_{10}, \Sigma_{10}\right)$ are exactly the complements of the cuts 
of $K_{5}$ (see Figure 1.5 for the corresponding labelling of $K_{5}$ ). For example, the complement of cut $\delta(\{C\})$ is $C_{1}=\left\{e_{1}, e_{2}, e_{3}, e_{4}, e_{5}, e_{6}\right\}$ and the complement of cut $\delta(\{B, D\})$ is $C_{2}=$ $\left\{e_{1}, e_{6}, e_{7}, e_{8}\right\}$. Note that both $C_{1}$ and $C_{2}$ correspond to minimally dependent column sets of the matrix representation, and both $C_{1}$ and $C_{2}$ intersect $\Sigma_{10}$ an odd number of times.

$$
\left[\begin{array}{llllllllll}
1 & 1 & 0 & 0 & 0 & 0 & 0 & 1 & 1 & 0 \\
1 & 0 & 1 & 0 & 0 & 0 & 0 & 1 & 0 & 1 \\
1 & 0 & 0 & 1 & 0 & 0 & 1 & 0 & 1 & 0 \\
1 & 0 & 0 & 0 & 1 & 0 & 1 & 0 & 0 & 1 \\
1 & 0 & 0 & 0 & 0 & 1 & 1 & 1 & 1 & 1
\end{array}\right], \Sigma_{10}=\left\{e_{1}, e_{7}, e_{8}\right\}
$$

Figure 1.6: Matrix and representation for $T_{10}$.

It can be verified that the unit weight function is Eulerian and satisfies the cut condition. A theorem of Seymour [30] shows that $\left(T_{10}, \Sigma_{10}\right)$ has no fractional flow. To prove this directly, the argument is similar to that for odd- $K_{5}$. A proof also arises from a relationship between fractional flow in a matroid and fractional flow in its dual (see Theorem 3.1.1).

\section{Lines of Fano Plane}

The binary matroid $F_{7}$ is rank 3 matroid with matrix representation given by Figure 1.7. The length 3 circuits of $F_{7}$ correspond to the lines of the Fano plane; for example $\left\{e_{1}, e_{5}, e_{7}\right\}$ is the length 3 circuit corresponding to the vertical line of the Fano plane. Let signature $\Sigma_{7}$ be any length 3 circuit of $F_{7}$; here we arbitrarily choose $\left\{e_{1}, e_{5}, e_{7}\right\}$.

$$
\left[\begin{array}{lllllll}
1 & 0 & 0 & 1 & 0 & 1 & 1 \\
0 & 1 & 0 & 1 & 1 & 0 & 1 \\
0 & 0 & 1 & 0 & 1 & 1 & 1
\end{array}\right], \Sigma_{7}=\left\{e_{1}, e_{5}, e_{7}\right\}
$$

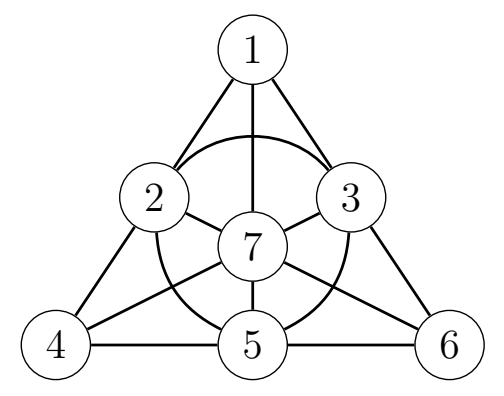

Figure 1.7: Matrix representation for $F_{7}$; length 3 odd circuits are exactly the lines of Fano plane (on right). 
Consider the multi-commodity flow instance for signed matroid $\left(F_{7},\left\{e_{1}, e_{5}, e_{7}\right\}\right)$ with unit weights. To verify the cut condition, we first note that the cocircuits of $F_{7}$ are exactly the complements of the lines of the Fano plane. Since for any cocircuit $D,\left|D \cap\left\{e_{1}, e_{5}, e_{7}\right\}\right| \leq$ 2 and $|D|=4$, the cut condition holds. Moreover, since all cocycles have an even number of elements, the weights are Eulerian. However, any circuit containing exactly one of $\left\{e_{1}, e_{5}, e_{7}\right\}$ has length exactly 3 and so to satisfy $\epsilon$ units of demand requires $2 \epsilon$ units of capacity. Since $w\left(\Sigma_{7}\right)=3,6$ total units of capacity are required, but only $w\left(E \backslash \Sigma_{7}\right)=4$ units are available. Thus $\left(F_{7}, \Sigma_{7}\right)$ has no fractional $\mathbb{1}$-flow and hence also no integer $\mathbb{1}$-flow. Resigning on cocircuit $\left\{e_{2}, e_{3}, e_{4}, e_{6}\right\}$ shows $E\left(F_{7}\right)$ is a signature of $\left(F_{7}, \Sigma_{7}\right)$.

\section{Postman Sets of Petersen Graph}

A postman set of graph $G=(V, E)$ is $S \subseteq E$ such that the odd degree vertices of $G[S]$ exactly coincide with the odd degree vertices of $G$. In Figure 1.8, an example of a postman set of the Petersen graph is given. Let $\left(P_{15}, \Sigma_{15}\right)$ be the signed matroid whose odd circuits are exactly the postman sets of the Petersen graph. Signed matroid $\left(P_{15}, \Sigma_{15}\right)$ has no fractional $\mathbb{1}$-flow. One way to show this is by deriving a connection between fractional flows and fractional colouring; there is no 3-colouring of the Petersen graph, but there is a fractional 3-colouring. Since colourings correspond to flows, $\left(P_{15}, \Sigma_{15}\right)$ has a fractional $\mathbb{1}$-flow but no integer $\mathbb{1}$-flow for the Eulerian unit weight function. For more information about connections between graph colouring and flows, see Section 2.4.4.

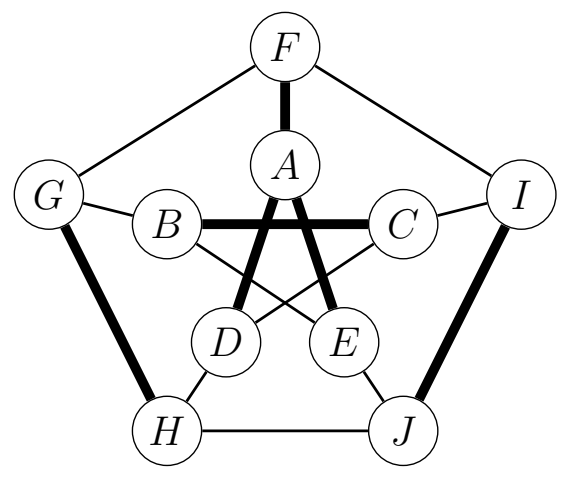

Figure 1.8: Graph is Petersen Graph; thick edges are an example of a postman set. 


\subsubsection{Minors}

The idea of using minors to characterize graphs extends to matroids. Let $M$ be a matroid; the two minor operations that can be applied to $M$ are deletion and contraction. The matroid obtained by deleting edge $e$ is denoted by $M \backslash e$ and has circuit set $\{C: C$ is a circuit of $\mathrm{M}$ with $e \notin C\}$. The matroid obtained by contracting edge $e$ is denoted by $M / e$ and has cycle set $\{C-e: C$ is a cycle of $\mathrm{M}\}$. A minor of $M$ is any matroid $M^{\prime}$ obtained by a sequence of contractions and deletions. Deletions and contractions can be performed in any order; if $M^{\prime}$ is obtained from $M$ by deleting edges in $I$ and contracting edges in $J$ in some order, then we can unambiguously denote $M^{\prime}$ by $M / I \backslash J$. One way to classify a matroid is according to whether it contains some fixed minor; $M$ contains $M^{\prime}$ as a minor if and only if there exists $I, J \subseteq E(M)$ such that $M^{\prime}=M / I \backslash J$.

Minors in matroids can be extended to minors in signed matroids. There are three minor operations: resigning, deletion and contraction. Resigning is done by replacing $\Sigma$ by $\Sigma^{\prime}=\Sigma \triangle D$ for $D$ a cocyle. Given $(M, \Sigma)$ and $e \in E$, we define $(M, \Sigma) \backslash e$ as $(M \backslash e, \Sigma-e)$ and call this operation deletion. Given $(M, \Sigma)$ and $e \in E$ that is not an odd loop ( $e$ is a loop if $\{e\}$ is dependent), suppose we resign to obtain $\Sigma^{\prime}$ such that $e \notin \Sigma^{\prime}$. We define $(M, \Sigma) / e$ as $\left(M / e, \Sigma^{\prime}\right)$ and call this operation contraction. A minor of $(M, \Sigma)$ is any signed matroid $\left(M^{\prime}, \Sigma^{\prime}\right)$ obtained from $(M, \Sigma)$ by a sequence of contractions and deletions.

Deleting edge $e$ removes all the circuits using $e$ and contracting edge $e$ shortens all the circuits using $e$ while maintaining their parity. Since minor operations can be applied in any order, we denote by $(M, \Sigma) / I \backslash J$ the signed matroid obtained by contracting all edges in $I \subseteq E$ and deleting all edges in $J \subseteq E$ (and resigning in whatever way avoids contracting signature edges). Necessarily $J$ does not contain an odd circuit. One way to classify a signed matroid is according to whether it contains a certain fixed minor; $(M, \Sigma)$ contains $\left(M^{\prime}, \Sigma^{\prime}\right)$ as a minor if and only if there exists $I, J \subseteq E(G)$ such that $\left(M^{\prime}, \Sigma^{\prime \prime}\right)=(M, \Sigma) / I \backslash J$ and $\Sigma^{\prime}=\Sigma^{\prime \prime} \triangle D$ for some cocyle $D$ of $\left(M^{\prime}, \Sigma^{\prime \prime}\right)$. Note that this definition does not account explicitly for the resigning operation. If $\left(M^{\prime}, \Sigma^{\prime}\right)$ is a minor of $(M, \Sigma)$ then so is any resigning of $\left(M^{\prime}, \Sigma^{\prime}\right)$. In other words, writing " $\left(M^{\prime}, \Sigma^{\prime}\right)$ is a minor of $(M, \Sigma)$ " means that every resigning of $\left(M^{\prime}, \Sigma^{\prime}\right)$ is a minor of every resigning of $(M, \Sigma)$.

\subsubsection{Existing Results and Conjectures}

Having defined matroid minors, we return to sufficient conditions for the existence of flows. Theorem 1.1.2 states graphs with no odd- $K_{4}$ minor always have integer flows. This theorem extends to integer flows in binary matroids. 
Theorem 1.2.2 (Seymour). Suppose $(M, \Sigma)$ has no odd- $K_{4}$ minor. Then for every $w \in \mathbb{Z}_{+}^{E}$ satisfying the cut condition, $(M, \Sigma)$ has an integer $w$-flow.

Generalization of Theorems 1.1.3 and 1.1.4 regarding fractional flows and Eulerian integer flows are not known. The Flowing Conjecture [30] posits that the bad instances previously discussed are precisely the obstructions to having a fractional flow for every weight function satisfying the cut condition. The Cycling Conjecture [30] posits that the bad instances previously discussed are precisely the obstructions to having an integer flow for every Eulerian weight function satisfying the cut condition.

Conjecture 1.2.3 (Flowing Conjecture). If signed matroid $(M, \Sigma)$ has no odd- $K_{5},\left(T_{10}, \Sigma_{10}\right)$, or $\left(F_{7}, E\left(F_{7}\right)\right)$ minor, then $M$ has a fractional $w$-flow for every $w \in \mathbb{Z}_{+}^{E}$ satisfying the cut condition.

Conjecture 1.2.4 (Cycling Conjecture). If signed matroid $(M, \Sigma)$ has no odd- $K_{5},\left(T_{10}, \Sigma_{10}\right)$, $\left(F_{7}, E\left(F_{7}\right)\right)$, or $\left(P_{15}, \Sigma_{15}\right)$ minor, then $M$ has an integer $w$-flow for every Eulerian $w \in \mathbb{Z}_{+}^{E}$ satisfying the cut condition.

Although the Cycling and Flowing Conjectures are open for general matroids, they are solved for some specific classes of matroids. For example, the Flowing Conjecture has been solved for lifts of graphic and cographic matroids whenever $|\Sigma|=1$.

\subsubsection{Lifts and Projections of Graphic and Cographic Matroids}

Consider binary matroid $M$ represented by 0,1 -matrix $A$. Let $A^{\prime}$ be obtained from $A$ by adding some 0,1 -row outside the row space of $A$. The binary matroid $M^{\prime}$ with representation $A^{\prime}$ is called a lift of $M$. Let $A^{\prime \prime}$ be obtained from $A$ by removing some row. Binary matroid $M^{\prime \prime}$ with representation $A^{\prime \prime}$ is called a projection of $M$.

Lifts of graphic matroids are known as even cycle matroids and lifts of cographic matroids are known as even cut matroids [25]. The Flowing Conjecture (Conjecture 1.2.3)is known to hold for lifts of graphic and cographic matroids whenever $\Sigma$ consists of exactly one edge [19].

Theorem 1.2.5. Let $M$ be a lift of a graphic matroid and $e \in E(M)$. If $(M,\{e\})$ has no odd- $K_{5}$ or $\left(F_{7}, E\left(F_{7}\right)\right)$ minor, then $M$ has a fractional $w$-flow for every $w \in \mathbb{Z}_{+}^{E}$ satisfying the cut condition. 
Theorem 1.2.6. Let $M$ be a lift of a cographic matroid and $e \in E(M)$. If $(M,\{e\})$ has no $\left(T_{10}, \Sigma_{10}\right)$ or $\left(F_{7}, E\left(F_{7}\right)\right)$ minor, then $M$ has a fractional $w$-flow for every $w \in \mathbb{Z}_{+}^{E}$ satisfying the cut condition.

It can be shown that the dual of a lift of $M$ is a projection of $M^{*}$. The Flowing Conjecture (Conjecture 1.2.3) also holds for projections of graphic and cographic matroids. For more details see Section 2.3.

\subsection{Results in This Thesis}

\subsubsection{Algorithmic Point of View}

Up to this point, we have been concerned with existential results. Given signed matroid $(M, \Sigma)$, and $w$ satisfying the cut condition,

1. Does $(M, \Sigma)$ have a fractional $w$-flow?

2. Does $(M, \Sigma)$ have an integer $w$-flow?

3. If $w$ is Eulerian, does $(M, \Sigma)$ have an integer $w$-flow?

Existential questions can be extended to questions about finding $w$-flows: given $(M, \Sigma)$ and $w \in \mathbb{Z}_{+}^{E(M)}$, the aim is to either

1. find a $w$-flow, or

2. show the cut condition is violated, or

3. find an obstruction.

Here, by obstruction we mean one of the signed graphs appearing in Theorem 1.2.2 and Conjectures 1.2.3 and 1.2.4. Our interest is in finding flows, obstructions, or cut condition violations "efficiently".

\subsubsection{Measuring Efficiency}

Given some problem with input $X$, we let $\langle X\rangle$ denote the encoding length of the input. For example, the encoding length of a rational number $\alpha=\frac{p}{q}$, is the total number of bits needed to represent both $p$ and $q$. The encoding length $\langle w\rangle$ of rational vector $w$ is the total encoding length of all entries of $w$. The encoding length $\langle A\rangle$ of matrix $A$ is the total encoding length of all entries of $A$. We consider an algorithm for problem with input $X$ efficient if it runs in time polynomial in $\langle X\rangle$. 
The input for a flow problem is $(M, \Sigma)$ and $w \in \mathbb{Z}_{+}^{E(M)}$. The size of the input depends on its representation; for example, matroid $M$ can be provided by its matrix representation $A$ or by a list of its circuits. Since the number of circuits may be exponential in the size of $A$, an algorithm running in polynomial time in the size of the circuit representation will not necessarily run in polynomial time in $\langle A\rangle$. Accordingly, we need to adopt some convention for measuring the size of a matroid. Throughout this paper, suppose $A$ is the smallest matrix representation of matroid $M$. Then $\langle A\rangle$ is the size of $M$. If $M$ is binary, then the entries of $A$ are 0 or 1 and so if $A$ is $m \times n$, then $\langle A\rangle=m n$.

For more details regarding efficiency measures and algorithm analysis see Grötschel et. al. ([16] Chapter 1.2). It is well-known the whenever the cut condition holds, the fractional multi-commodity flow problem can be solved in polynomial time using linear programming. The integer multi-commodity flow problem is polynomial time solvable for $|\Sigma|=1$ using network flow techniques. However, this problem is known to be NP-hard for $|\Sigma| \geq 2[10]$.

\subsubsection{Algorithms Arising from Existential Results}

Although we cannot hope for a polynomial time algorithm for solving arbitrary flow instances, restricting the instances in consideration may allow for efficient algorithms. One natural class of problems to consider arises from existential results; does knowing that $(M, \Sigma)$ has a integer (or fractional) flow for every $w \in \mathbb{Z}_{+}^{E}$ satisfying the cut condition help in efficiently finding flows?

By Seymour's Theorem, the instances with integer flows are exactly the instances for which $(M, \Sigma)$ has no odd- $K_{4}$ minor. Using a decomposition theorem, Truemper [33] shows this result leads to an algorithm.

Theorem 1.3.1. Let $(M, \Sigma)$ be a signed binary matroid with matrix representation $A$ and let $w \in \mathbb{Z}_{+}^{E(M)}$ be some weight function. Then in time polynomial in $\langle A\rangle$ and $\langle w\rangle$, one can either

1. show the cut condition is violated, or

2. find $I, J \subseteq E(M)$ such that $(M, \Sigma) / I \backslash J$ is odd- $K_{4}$, or

3. find an integer $w$-flow.

Note that the above result is (in some sense) stronger than simply being able to find an integer $w$-flow whenever the cut condition is satisfied and $(M, \Sigma)$ has no odd- $K_{4}$ minor. Given an arbitrary matroid, this results shows that we can either find an integer $w$-flow or provide a certificate as to why we could not. 
Since the Flowing Conjecture (Conjecture 1.2.3) is open for general clutters, we cannot hope to prove a fractional analogue of Theorem 1.3.1. However, in Chapter 4 of this thesis, we show that the analogous result holds whenever $M$ is the lift of a graphic or cographic matroid and $|\Sigma|=1$.

Theorem 1.3.2. Let $M$ with representation $A$ be the lift of a graphic matroid and $e \in$ $E(M)$. Consider the flow instance on signed matroid $(M,\{e\})$ with weight function $w \in$ $\mathbb{Z}_{+}^{E}$. Then in time polynomial in $\langle A\rangle$ and $\langle w\rangle$ one can either

1. show the cut condition is violated, or

2. find $I, J \subseteq E(M)-e$ such that $(M,\{e\}) / I \backslash J$ is odd- $K_{5}$ or $\left(F_{7}, E\left(F_{7}\right)\right)$, or

3. find a fractional $w$-flow.

Theorem 1.3.3. Let $M$ with representation $A$ be the lift of a cographic matroid and $e \in$ $E(M)$. Consider the flow instance on signed matroid $(M,\{e\})$ with weight function $w \in$ $\mathbb{Z}_{+}^{E}$. Then in time polynomial in $\langle A\rangle$ and $\langle w\rangle$ one can either

1. show the cut condition is violated, or

2. find $I, J \subseteq E(M)-e$ such that $(M,\{e\}) / I \backslash J$ is $\left(T_{10}, \Sigma_{10}\right)$ or $\left(F_{7}, E\left(F_{7}\right)\right)$, or

3. find a fractional $w$-flow.

It follows from the extension of the graph minors project to binary matroids that the problem of checking for the presence of the aforementioned obstructions can be done in polynomial time. Hence an existing algorithm for this problem would be to first check for any obstruction and then, if none exists, simply solve the covering linear program to obtain a necessarily integral solution and, hence, a cover. However, the algorithms arising from the matroid minor project are both complicated and impractical because they arise from Ramsey-type arguments and hence have astronomical constants.

The algorithms presented herein use as a tool a theorem of Lehman characterizing minimally non-ideal clutters. Although Lehman's Theorem was non-constructive and applied to minimally non-ideal clutters, a constructive variant can be shown. This is the topic of Chapter 3. As Lehman's Theorem was the basis for many excluded minor results, this constructive version of Lehman's Theorem and a constructive spin on the related excluded minor results leads to the proofs of Theorems 1.3.2 and 1.3.3 appearing in Chapter 4.

An interesting corollary of Theorem 1.3.2 is that the NP-hard maximum cut problem can be solved in polynomial time for graphs that do not contain $K_{5}$ as an odd minor. We say $K_{n}$ is an odd minor of graph $G$ if it can be obtained by first deleting edges and then contracting edges on a cut. If $K_{n}$ is an odd minor of $G$, then it is a minor, but the converse is not true in general. 
Corollary 1.3.4. Let $G$ be a graph and let $w \in \mathbb{Z}_{+}^{E(G)}$. Then in time polynomial in $|V(G)|+\langle w\rangle$ we can either,

1. find a maximum weight cut of $G$, or

2. find $K_{5}$ as an odd minor of $G$.

\subsection{Overview of Remainder of Thesis}

Chapter 2 is a more in-depth coverage of flowing and cycling results; it introduces the related minimax equation and overviews the classes of matroids for which single commodity results are known. The chapter concludes by introducing clutters and showing the relationship to flows in matroids. Chapter 3 starts by introducing the necessary polyhedral background. The remainder of the chapter is dedicated to introducing and proving a constructive version of Lehman's Theorem. Chapter 4 shows how the constructive version of Lehman's Theorem can be applied to lifts of graphic and cographic matroids to prove Theorems 1.3.2 and 1.3.3. Chapter 5 concludes this thesis and addresses future areas of work, including solving Eulerian integer multi-commodity flow in a naturally arising class of signed graphs. 


\section{Chapter 2}

\section{Flow Results and Relationship to Clutters}

\subsection{Flows as a Minimax Relation}

\subsubsection{Definitions and Connections}

Given multi-commodity flow instance $(M, \Sigma, w)$, let $\mathcal{C}$ denote the set of odd circuits of $(M, \Sigma)$. Consider the following primal-dual pair.

$$
\begin{array}{rlr}
\min & \sum\left(w_{e} x_{e}: e \in E\right) & \\
\text { subject to } & \sum\left(x_{e}: e \in C\right) \geq 1 \quad \text { for all } C \in \mathcal{C} \\
& & \\
\max & \sum\left(y_{C}: C \in \mathcal{C}\right) & \\
\text { subject to } & \sum\left(y_{C}: e \in C\right) \leq w_{e} & \text { for all } e \in E \backslash \Sigma \\
& y \geq \mathbb{O} . &
\end{array}
$$

The program (2.1) models the problem of finding the minimum weight fractional cover. Any integral solution to $(2.1)$ is a cover of $(M, \Sigma)$. The minimal covers of $(M, \Sigma)$ are exactly 
the signatures of $(M, \Sigma)$. We let $\tau_{w}^{*}(M, \Sigma)$ denote the fractional optimal solution to (2.1) and $\tau_{w}(M, \Sigma)$ denote the integer optimal solution. The program (2.2) models the problem of packing odd circuits. A solution to program (2.2) is called a fractional w-packing of odd circuits of $(M, \Sigma)$. Any integral solution to $(2.2)$ is called an integer w-packing of odd circuits of $(M, \Sigma)$. We let $\nu_{w}^{*}(M, \Sigma)$ denote the fractional optimal solution to $(2.2)$ and $\nu_{w}(M, \Sigma)$ denote the integer optimal solution. The following relationship follows directly from LP duality.

Remark 2.1.1. $\tau_{w}(M, \Sigma) \geq \tau_{w}^{*}(M, \Sigma)=\nu_{w}^{*}(M, \Sigma) \geq \nu_{w}(M, \Sigma)$.

The existence of flows in signed matroids corresponds to packings and covers of equal value. The following two remarks make this statement precise.

Proposition 2.1.2. If $w \in \mathbb{Z}_{+}^{E}$ satisfies the cut condition, then $(M, \Sigma)$ has a fractional $w$-flow if and only if $\tau_{w}(M, \Sigma)=\nu_{w}^{*}(M, \Sigma)$.

Proof. Since $w$ satisfies the cut condition, $\Sigma$ is a minimum weight signature by Remark 1.2.1. If $(M, \Sigma)$ has fractional $w$-flow $y$, then $y$ is a fractional packing of odd circuits of $(M, \Sigma)$ of value $w(\Sigma)$. That is $\tau_{w}(M, \Sigma)=\nu_{w}^{*}(M, \Sigma)$.

For the other direction, suppose $\tau_{w}(M, \Sigma)=\nu_{w}^{*}(M, \Sigma)$. This means (2.1) has integral optimal solution of value $w(\Sigma)$ and (2.2) has fractional optimal solution of value $w(\Sigma)$. By complementary slackness,

1. for each $C \in \mathcal{C}$, if $y_{C}>0$, then $|C \cap \Sigma|=1$, and,

2. for each $d \in \Sigma, \sum\left(y_{C}: e \in C \in \mathcal{C}_{d}\right)=w_{d}$.

Let $\mathcal{C}_{1}$ again denote the set of odd circuits of $(M, \Sigma)$ intersecting $\Sigma$ in exactly one edge. The restriction of $y$ to $\mathcal{C}_{1}$ is a fractional $w$-flow for $(M, \Sigma)$.

Proposition 2.1.3. If $w \in \mathbb{Z}_{+}^{E}$ satisfies the cut condition, then $(M, \Sigma)$ has a integer $w$-flow if and only if $\tau_{w}(M, \Sigma)=\nu_{w}(M, \Sigma)$.

Proof. Analogous to proof of Proposition 2.1.2.

Note that if $\tau_{w}(M, \Sigma)=\nu_{w}(M, \Sigma)$ for all $w \in \mathbb{Z}_{+}^{E}$, then $\tau_{w}\left(M^{\prime}, \Sigma^{\prime}\right)=\nu_{w}\left(M^{\prime}, \Sigma^{\prime}\right)$ for every minor $\left(M^{\prime}, \Sigma^{\prime}\right)$ of $(M, \Sigma)$. This follows since deletion corresponds to setting the weight of an edge to 0 and contraction corresponds to setting the weight of an edge to some large number (effectively $\infty$ ). Seymour's Theorem (Theorem 1.2.2) regarding integer flows and the Flowing and Cycle Conjectures (Conjectures 1.2.3 and 1.2.4) can be restated as follows. 
Theorem 2.1.4 (Seymour). The equation $\tau_{w}(M, \Sigma)=\nu_{w}(M, \Sigma)$ holds for all $w \in \mathbb{Z}_{+}^{E}$ if and only if $(M, \Sigma)$ has no odd- $K_{4}$ minor.

Conjecture 2.1.5 (Flowing Conjecture). The equation $\tau_{w}(M, \Sigma)=\nu_{w}^{*}(M, \Sigma)$ holds for all $w \in \mathbb{Z}_{+}^{E}$ if and only if $(M, \Sigma)$ has no odd- $K_{5},\left(T_{10}, \Sigma_{10}\right)$ or $\left(F_{7}, E\left(F_{7}\right)\right)$ minor.

Conjecture 2.1.6 (Cycling Conjecture). The equation $\tau_{w}(M, \Sigma)=\nu_{w}(M, \Sigma)$ holds for all Eulerian $w \in \mathbb{Z}_{+}^{E}$ if and only if $(M, \Sigma)$ has no odd- $K_{5},\left(T_{10}, \Sigma_{10}\right),\left(F_{7}, E\left(F_{7}\right)\right)$ or $\left(P_{15}, \Sigma_{15}\right)$ minor.

For unit weights, finding an integer packing is equivalent to finding pairwise disjoint odd circuits of $(M, \Sigma)$. This leads to combinatorial interpretations of the excluded minor results. The next two sections investigate Seymour's Theorem (Theorem 2.1.4) and the Cycling Conjecture (Conjecture 2.1.6) for graphic and cographic matroids.

\subsubsection{Application to Graphic Matroids}

Suppose $M$ is the graphic matroid corresponding to graph $G$. As indicated in Section 1.1.4, the Cycling Conjecture (Conjecture 2.1.6) holds for graphic matroids. The odd circuits of $(M, \Sigma)$ are the circuits of $G$ intersecting $\Sigma$ an odd number of times. Considering unit weights, Seymour's Theorem (Theorem 2.1.4) and the Cycling Conjecture (Conjecture 2.1.6) imply the following minimax relationships.

Corollary 2.1.7. If $(M, \Sigma)$ has no odd- $K_{4}$ minor, then the minimum size of a signature of $(G, \Sigma)$ is equal to the maximum number of pairwise disjoint odd circuits in $(G, \Sigma)$.

Corollary 2.1.8. If $(M, \Sigma)$ has no odd- $K_{5}$ minor and $|\delta(\{v\})|$ is even for all $v \in V(G)$, then the minimum size of a signature of $(G, \Sigma)$ is equal to the maximum number of pairwise disjoint odd circuits in $(G, \Sigma)$.

\subsubsection{Application to Cographic Matroids}

Suppose $M$ is the cographic matroid corresponding to graph $G$. A result of Seymour [29] shows the Cycling Conjecture (Conjecture 2.1.6) holds for cographic matroids. Given signature $\Sigma$, let $T$ denote the odd degree vertices of $G[\Sigma]$. Then the odd circuits of $(M, \Sigma)$ are the $T$-cuts of $(G, T)$. A $T$-cut is $\delta(S)$ for $S \subseteq V$ such that $|S \cap T|$ is odd. The covers of $(M, \Sigma)$ are the $T$-joins of $(G, T)$. A $T$-join is $J \subseteq E$ such that the odd vertices $G[J]$ are $T$. 
Odd- $K_{4}$ has one realization for $M$ cographic (see Figure 2.1). None of the obstructions appearing in the Cycling Conjecture (Conjecture 2.1.6) have a realization in which $M$ is cographic. If the unit weight function is Eulerian, then every cycle of $G$ has even length. Combining these observations, Seymour's Theorem (Theorem 2.1.4) and the Cycling Conjecture (Conjecture 2.1.6) reduce to the following minimax relationships.

Theorem 2.1.9. Suppose $M$ is the cographic matroid corresponding to $G$ and $\Sigma \subseteq E(G)$ is such that $T$ denotes the odd vertices of $G[\Sigma]$. If $(M, \Sigma)$ has no odd- $K_{4}$ minor, then the size of a minimum of a $T$-join of $(G, T)$ is equal to the maximum number of pairwise disjoint $T$-cuts of $(G, T)$.

Theorem 2.1.10 (Seymour). If $(G, \Sigma)$ is bipartite and $\Sigma \subseteq E(G)$ is such that $T$ denotes the odd vertices of $G[\Sigma]$, then the size of a minimum $T$-join of $(G, T)$ is equal to the maximum number of pairwise disjoint $T$-cuts of $(G, T)$.

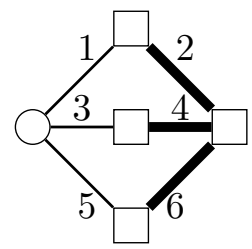

Figure 2.1: Cographic Odd- $K_{4}$ : Square vertices are $T$ vertices.

\subsection{Multi-Commodity to Single Commodity Flow}

\subsubsection{Equivalence}

If $\Sigma=\{e\}$, then $(M, \Sigma)$ and $w \in \mathbb{Z}_{+}^{E}$ specify a single commodity flow instance. There is "no difference" between single and multi-commodity flow for the class of all binary matroids.

Let $(M, \Sigma)$ be a signed matroid and assume $e \notin E(M)$. Let $\mathcal{C}_{\text {odd }}$ denote the set of odd circuits of $(M, \Sigma)$ and $\mathcal{C}_{\text {even }}$ denote the set of even circuits of $(M, \Sigma)$. Let matroid $M^{\prime}$ have circuit set $\left\{C: C \in \mathcal{C}_{\text {even }}\right\} \cup\left\{C \cup\{e\}: C \in \mathcal{C}_{\text {odd }}\right\}$. We call $M^{\prime}$ the port representation of $(M, \Sigma)$ with port $e$. By construction, the circuits of $M^{\prime}$ containing edge $e$ are exactly the odd circuits of $M$. The first remark below shows that $M^{\prime}$ is indeed a binary matroid. The second remark shows that multi-commodity flow in $(M, \Sigma)$ corresponds to single commodity flow in $\left(M^{\prime},\{e\}\right)$. 
Remark 2.2.1. Let $M^{\prime}$ be a port representation of signed binary matroid $(M, \Sigma)$. Then $M^{\prime}$ is a binary matroid.

Proof. Let $B$ be a matrix representation of $M^{*}$; the rows of $B$ are characteristic vectors of cycles of $M$. Obtain $B^{\prime}$ by appending a column to $B$ that has an 1 in position $i$ if and only if the cycle corresponding to row $i$ of $B$ as an odd cycle of $(M, \Sigma)$. It can be readily checked that the matroid whose cycle space is spanned by the rows of $B^{\prime}$ is exactly $M^{\prime}$.

Remark 2.2.2. Let $M^{\prime}$ be a port representation of signed binary matroid $(M, \Sigma)$ with port e. Let $w^{\prime} \in \mathbb{Z}_{+}^{E(M)}$ be the weight function defined by $w_{f}^{\prime}=w_{f}$ for all $f \in E(M)$ and $w_{e}^{\prime}=w(\Sigma)$. Then

1. $(M, \Sigma, w)$ satisfies the cut condition if and only if $\left(M^{\prime},\{e\}, w^{\prime}\right)$ satisfies the cut condition.

2. $(M, \Sigma)$ has a fractional $w$-flow if and only if $\left(M^{\prime},\{e\}\right)$ has a fractional $w^{\prime}$-flow.

3. $(M, \Sigma)$ has an integer $w$-flow if and only if $\left(M^{\prime},\{e\}\right)$ has an integer $w^{\prime}$-flow.

It follows we can restrict ourselves to single commodity flow when considering the class of all binary matroids. However, we cannot use this restriction for fixed classes of binary matroids. For example, suppose we are interested in multi-commodity flow instances in graphic matroids. There is no integer flow whenever $(M, \Sigma)$ has an odd- $K_{4}$ minor. However, the Ford Fulkerson Max Flow Min Cut Theorem [11] shows that any graphic single commodity flow instance has an integer flow whenever the cut condition is satisfied. Switching from multi-commodity flow in graphic $M$ to single commodity flow in $M^{\prime}, M^{\prime}$ will not necessarily be graphic. In fact, for $(M, \Sigma)=$ odd- $K_{4}, M^{\prime}$ cannot be graphic.

To illustrate Remark 2.2.2, consider the multi-commodity flow instance $\left(F_{7}, \Sigma_{7}, w\right)$. It is straightforward to verify that $A G(3,2)$ (with matrix representation given in Figure $2.2)$ is the port representation of $\left(F_{7}, \Sigma_{7}\right)$ with port $e_{8}$. In particular, the circuits of $A G(3,2)$ using edge $e_{8}$ are exactly the odd circuits of $\left(F_{7}, \Sigma_{7}\right)$ (which are exactly the odd circuits of $\left(F_{7}, E\left(F_{7}\right)\right)$. Finding a $w$-flow for $\left(F_{7}, \Sigma_{7}\right)$ is equivalent to finding a $w^{\prime}$-flow for $\left(A G(3,2),\left\{e_{8}\right\}\right)$ where $w_{f}^{\prime}=w_{f}^{\prime}$ for all $f \in E-e_{8}$ and $w_{e_{8}}^{\prime}=w\left(\Sigma_{7}\right)$.

It can be shown that $T_{11}$ is a port representation of $\left(T_{10}, \Sigma_{10}\right)$ with port $e_{11}$ and that $T_{11}^{*}$ is a port representation of odd- $K_{5}$ with port $e_{11}$. For a matrix representation of $T_{11}$, see Figure 2.3. 


$$
\left[\begin{array}{llllllll}
1 & 0 & 0 & 0 & 1 & 0 & 1 & 1 \\
0 & 1 & 0 & 0 & 0 & 1 & 1 & 1 \\
0 & 0 & 0 & 1 & 1 & 1 & 0 & 1 \\
0 & 0 & 1 & 0 & 1 & 1 & 1 & 0
\end{array}\right]
$$

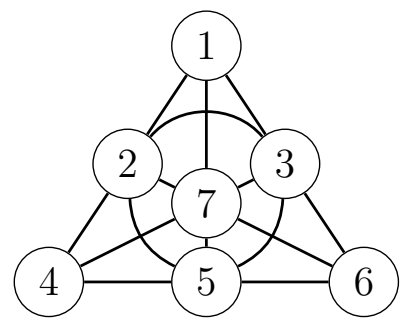

Figure 2.2: Matrix representation for $A G(3,2)$ (left); circuits using $e_{8}$ (element represented by the 8th column) correspond to lines of Fano plane (right).

$$
T_{11}:\left[\begin{array}{ccccccccccc}
1 & 0 & 0 & 0 & 0 & 0 & 1 & 1 & 0 & 0 & 1 \\
0 & 1 & 0 & 0 & 0 & 0 & 1 & 0 & 1 & 0 & 1 \\
0 & 0 & 1 & 0 & 0 & 0 & 1 & 0 & 0 & 1 & 1 \\
0 & 0 & 0 & 1 & 0 & 0 & 0 & 1 & 1 & 0 & 1 \\
0 & 0 & 0 & 0 & 1 & 0 & 0 & 1 & 0 & 1 & 1 \\
0 & 0 & 0 & 0 & 0 & 1 & 0 & 0 & 1 & 1 & 1
\end{array}\right]
$$

Figure 2.3: Matrix representation for $T_{11}$

\subsubsection{Reformulating Single Commodity Flow Using e-Paths}

Given matroid $M$ and element $e \in E(M)$, an $e$-path is a set of the form $C-e$ where $C$ is a circuit of $M$ containing $e$. Let $\mathcal{F}$ denote the set of $e$-paths of $M$. Given $w \in \mathbb{Z}_{+}^{E-e}$, we call $\mathcal{P} \subseteq \mathcal{F}$ a $w$-packing of $e$-paths if $|\{P \in \mathcal{P}: f \in P\}| \leq w_{f}$ for all $f \in E$.

An $e$-cut is a minimal subset of ground set $E$ that intersects every e-path; that is, $U \subseteq E$ is an $e$-cut if $|U \cap P| \geq 1$ for all $e$-paths $P$ and if $U^{\prime} \subseteq U$ is such that $\left|U^{\prime} \cap P\right| \geq 1$ for all $e$-paths $P$ then $U^{\prime}=U$. Whenever $M$ is binary, the $e$-cuts of $M$ are exactly the $e$-paths of $M^{*}$. A proof of this fact will appear later (see Fact 2.5.2).

Given $M, e$ and $w$, consider the following primal-dual pair of linear programs. 


$$
\begin{array}{rlr}
\min & \sum\left(w_{f} x_{f}: f \in E-e\right) & \\
\text { subject to } & \sum\left(x_{f}: f \in P\right) \geq 1 & \\
& x \geq \mathbb{O} & \\
\max & \sum\left(y_{P}: P \text { is an } e \text {-path }\right) & \\
\text { subject to } & \sum\left(y_{P}: f \in e \text {-path } P\right) \leq w_{f} & \text { for all } f \in \text {-paths } P \\
& y \geq \mathbb{O} . &
\end{array}
$$

Note that (2.4) corresponds exactly to (2.2) whenever $\Sigma=\{e\}$ and $w_{e}$ is maximal such that the cut condition holds. To obtain the correspondence, simply map $e$-path $P$ to its corresponding odd circuit $P \cup\{e\}$. Since $w_{e}$ is maximal, there exists an optimal cover for 2.3 that is distinct from $\{e\}$. Likewise (2.3) corresponds exactly to $(2.1)$ whenever $\Sigma=\{e\}$. It follows from Proposition 2.1.2 that we can solve single commodity flow instance $(M,\{e\}, w)$ by solving (2.4) to find a maximum packing of $e$-paths of $M$. In Section 2.5, this relationship will be useful to connect statements about flows to statements about clutters.

Recall that we are interested in signed matroids for which the minimax equations $\left(\tau_{w}=\right.$ $\left.\nu_{w}, \tau_{w}=\nu_{w}^{*}\right)$ hold at equality for all weight functions. We say $(M,\{e\})$ has the

1. fractional $M F M C$ property if for every $w \in \mathbb{Z}_{+}^{E}$ there is an integer solution to (2.3) and a solution to (2.4) with the same objective value,

2. integer $M F M C$ property if for every $w \in \mathbb{Z}_{+}^{E}$ there is an integer solution to (2.3) and an integer solution to (2.4) with the same objective value,

3. Eulerian integer $M F M C$ property if for every Eulerian $w \in \mathbb{Z}_{+}^{E}$ there is an integer solution to (2.3) and an integer solution to (2.4) with the same objective value.

Seymour's Theorem (Theorem 1.2.2) and the Flowing and Cycling Conjectures (Conjectures 1.2 .4 and 1.2 .3$)$ can be restated using this terminology. Recall that $\left(P_{15}, \Sigma_{15}\right)$ is the signed binary matroid with odd circuits corresponding to the postman sets of the Petersen graph. Let $P_{16}$ be the port representation of $\left(P_{15}, \Sigma_{15}\right)$ with port $e_{16}$.

Theorem 2.2.3 (Seymour). (M, $\{e\})$ has the integer MFMC property if and only if it does not have a minor isomorphic to odd- $K_{4}$.

Conjecture 2.2.4 (Flowing Conjecture). (M, $\{e\}$ ) has the fractional MFMC property if and only if it does not have a minor isomorphic to $\left(A G(3,2),\left\{e_{8}\right\}\right),\left(T_{11},\left\{e_{11}\right\}\right)$, or $\left(T_{11}^{*},\left\{e_{11}\right\}\right)$. 
Conjecture 2.2.5 (Cycling Conjecture). ( $M,\{e\})$ has the Eulerian integer MFMC property if and only if it does not have a minor isomorphic to $\left(A G(3,2),\left\{e_{8}\right\}\right),\left(T_{11},\left\{e_{11}\right\}\right)$, $\left(T_{11}^{*},\left\{e_{11}\right\}\right)$, or $\left(P_{16},\left\{e_{16}\right\}\right)$.

The Flowing and Cycling Conjectures are solved for some classes of single commodity flow instances. For example, they are known to hold whenever $M$ is graphic or cographic.

\subsubsection{Single Commodity Flow for Graphic Matroids}

Consider a graph $G$ with distinct vertices $s, t$ and edge $e=(s, t)$. Let $H$ be obtained from $G$ by deleting $e$. Suppose $M$ is the graphic matroid corresponding to $G$. The $e$-paths and $e$-cuts of $M$ correspond respectively to the $s t$-paths and st-cuts of $H$. An integer solution to (2.6) gives an integer st-flow, and an integer solution to (2.5) gives the characteristic vector of an st-cut. By the Ford Fulkerson Max Flow Min Cut Theorem [11], the value of a maximum st-flow is equal to the size of a minimum st-cut. In other words, the integer MFMC property holds for $(M,\{e\})$ whenever $M$ is a graphic matroid.

\subsubsection{Single Commodity Flow for Cographic Matroids}

Consider a graph $G$ with distinct vertices $s, t$ and edge $e=(s, t)$. Let $H$ be obtained from $G$ by deleting $e$. Suppose $M$ is the cographic matroid corresponding to $G$. The $e$-paths and $e$-cuts of $M$ correspond to the st-cuts and st-paths of $H$ respectively. An integer solution to (2.6) gives an integer packing of st-cuts, and an integer solution to (2.5) gives the characteristic vector of an st-path. The Max Work Min Potential Theorem of Duffin [9] states that the size of a maximum packing of st-cuts is equal to the minimum length of an st-path. In other words, the integer MFMC property holds for $(M,\{e\})$ whenever $M$ is a cographic matroid.

\subsection{Lifts and Projections of Graphic and Cographic Matroids}

\subsubsection{Cycle and Cocycle Spaces for Binary Matroids}

Let $M$ be a binary matroid. The circuits of $M$ are exactly the sets of minimally linearly dependent columns for some matrix $A \in G F(2)^{m \times n}$. The cycles of $M$ are obtained by 
taking the symmetric difference of some finite number of circuits; any cycle $C$ can be partitioned into circuits $C_{1}, \ldots C_{k}$ such that $C=C_{1} \triangle \ldots \triangle C_{k}$. The set of cycles of $M$ is called the cycle space of the matroid. If $C_{1}$ and $C_{2}$ are cycles of $M$, then $C_{1} \triangle C_{2}$ is also a cycle of $M$. A set of cycles $\mathcal{C}^{\prime}$ spans the cycle space of $M$ if every cycle of $M$ can be expressed as the symmetric difference of cycles in $\mathcal{C}^{\prime}$. A basis for the cycle space of $M$ is any minimal set of cycles spanning the cycle space of $M$.

Consider matroid $M$ with matrix representation $A$. The cycle space of $M^{*}$ is called the cocycle space of $M$. It is spanned by the characteristic sets of the rows of $A$. (In the future, we will abuse notation and simply say the cycle space of $M$ is spanned by the row space of $A$; the meaning is clear.) Since $\left(M^{*}\right)^{*}=M$, if $B$ is a matrix representation for $M^{*}$, the row space of $B$ spans the cycle space of $M$. From Proposition 2.2.23 of [24], the row space of $\mathrm{A}$ is orthogonal to the row space of $B$ whenever $A$ is a binary matroid. It follows that if $x$ is the characteristic vector of a cycle of $M$ and $y$ is the characteristic vector of a cocycle of $M, x^{T} y=0$.

Consider binary matroid $M$ represented by 0,1 -matrix $A$. Let $A^{\prime}$ be obtained from $A$ by adding some 0,1 -row outside the row space of $A$. Recall that the binary matroid $M^{\prime}$ with representation $A^{\prime}$ is called a lift of $M$. Let $A^{\prime \prime}$ be obtained from $A$ by removing some row of $A$. Recall that the binary matroid $M^{\prime}$ with representation $A^{\prime}$ is called a projection of $M$.

Augmenting $A$ by row $x$ is the same as a projection of the cycle space of $M$ onto the

set $\left\{y: x^{T} y=0\right\}$. By Rank-Nullity, this corresponds to increasing the dimension of the cocycle space of $M$ by 1 . That is, if $M^{\prime}$ denotes the matroid with representation $A^{\prime}$, then the cycle space of $\left(M^{\prime}\right)^{*}$ has dimension one more than the dimension of the cycle space of $M^{*}$. In other words, the dual of a lift of $M$ is a projection of $M^{*}$.

\subsubsection{Even Cycle Matroids}

Even cycle matroids were introduced by Zaslavsky [35] and have a nice interpretation with respect to signed graphs. Given graph $G$, let $A$ be a matrix representation of $M(G)$. Let $A^{\prime}$ be obtained by augmenting $A$ with row $x^{T}$ outside the row space of $A$ where $x^{T}$ is the characteristic vector of $\Sigma \subseteq E$. The matroid with matrix representation $A^{\prime}$ is denoted by ecycle $(G, \Sigma)$ and called the even cycle matroid of $(G, \Sigma)$. The cycles of ecycle $(G, \Sigma)$ are exactly the even cycles of signed graph $(G, \Sigma)$. Moreover, the circuits of ecycle $(G, \Sigma)$ come in two types:

1. $C \subseteq E$ such that $C$ is an even circuit of $(G, \Sigma)$, and, 
2. $C_{1} \cup C_{2} \subseteq E$ such that $C_{1}$ and $C_{2}$ are both odd circuits of $(G, \Sigma)$ and share at most one vertex.

Let $D$ denote the dual of ecycle $(G, \Sigma)$. The cycle space of $D$ is spanned by the row space of $A^{\prime}$. The rows of $A^{\prime}$ are the characteristic vectors of cuts of $G$ and the characteristic vector of $\Sigma$. For signed graph $(G, \Sigma)$ : the symmetric difference of two cuts is a cut, the symmetric difference of two signatures is a cut, and the symmetric difference of a signature and a cut is a signature. It follows that the cycles of the $D$ are exactly the cuts and signatures of $(G, \Sigma)$.

\subsubsection{Even Cut Matroids}

Even cut matroids were also introduced by Zaslavsky [35] and have a nice interpretation with respect to grafts. Given graph $G$, let $A$ be a matrix representation of $M^{*}(G)$. Let $A^{\prime}$ be obtained by augmenting $A$ with row $x^{T}$ outside the row space of $A$ where $x^{T}$ is the characteristic vector of $\Sigma \subseteq E$. Let $T$ be the set of odd degree vertices in $G[\Sigma]$. Note that $|T|$ is even since any graph has an even number of odd degree vertices. The matroid with matrix representation $A^{\prime}$ is denoted by $\operatorname{ecut}(G, T)$ and called the even cut matroid of $(G, T)$. The cycles of ecut $(G, T)$ are exactly the T-even cuts of $G$; a cut $\delta(U)$ of $G$ is $T$-even if $|U \cap T|$ is even.

Let $D$ denote the dual of ecut $(G, T)$. The cycle space of $D$ is spanned by the row space of $A^{\prime}$. The rows of $A^{\prime}$ are the characteristic vectors of cycles of $G$ and the characteristic vector of $\Sigma$. A $T$-join is $F \subseteq E(G)$ such that the odd degree vertices of $G[F]$ are exactly $T$; since $T$ is the set of odd vertices of $G[\Sigma], \Sigma$ is a $T$-join. In graft $(G, T)$ : the symmetric difference of two cycles is a cycle, the symmetric difference of two $T$-joins is a cycle, and the symmetric difference of a cycle and a $T$-join is a $T$-join. It follows that the cycles of the dual of ecut $(G, T)$ are exactly the cycles and $T$-joins of $(G, T)$.

\subsection{Single Commodity Flow Results}

Here we discuss Seymour's Theorem (Theorem 2.2.3) and the single commodity Flowing and Cycling Conjectures (Conjectures 2.2.4 and 2.2.5) for lifts and projections of graphic and cographic matroids. 


\subsubsection{Even Cycle Matroids}

Let $M=\operatorname{ecycle}(G, \Sigma)$ for graph $G=(V, E)$ and $\Sigma \subseteq E$. For $e=s t \in \Sigma$, an $e$-path is either an odd st-path or the union of an even st-path and odd circuit sharing at most one vertex. The $e$-paths of an even cycle matroid are called odd-st-walks. If $s=t$, there are no odd-st-paths and all even st-paths have length zero; the $e$-paths of $M$ are exactly the odd circuits of $(G, \Sigma)$. Odd circuits have been studied extensively with respect to flows; see the next section (Section 2.4.2) for an overview of results. The single commodity Flowing Conjecture (Conjecture 2.2.4) is true for even cycle matroids [19], but the single commodity Cycling Conjecture (Conjecture 2.2.5) remains open.

Theorem 2.4.1. If $M$ is an even cycle matroid, then $(M,\{e\})$ has the fractional $M F M C$ property if and only if it has no $\left(T_{11}^{*},\left\{e_{11}\right\}\right)$ or $\left(A G(3,2),\left\{e_{8}\right\}\right)$ minor.

\subsubsection{Even Cycle Matroids: $e$ a loop}

The single commodity Flowing and Cycling Conjectures (Conjectures 2.2.4 and 2.2.5) are resolved for even cycle matroids whenever $e$ is a loop of $G$ [20] [13].

Theorem 2.4.2. If $M$ is an even cycle matroid and e is a loop of underlying graph $G$, then $(M,\{e\})$ has the fractional MFMC property if and only if it has no $\left(T_{11}^{*},\left\{e_{11}\right\}\right)$ minor.

Theorem 2.4.3. If $M$ is an even cycle matroid and e is a loop of underlying graph $G$, then $(M,\{e\})$ has the Eulerian integer MFMC property if and only if it has no $\left(T_{11}^{*},\left\{e_{11}\right\}\right)$ minor.

Although it may seem unintuitive to consider the case when $e$ is a loop, this case actually corresponds exactly to multi-commodity flow in graphs. Given $(G, \Sigma)$ and $w \in \mathbb{Z}_{+}^{E}$, let $H$ be obtained from $G$ by adding loop $e \in \Sigma$ and let $M=\operatorname{ecycle}(H, \Sigma)$. The $e$-paths of $M$ are exactly the odd circuits of $(G, \Sigma)$. Finding a packing of $e$-paths is equivalent to solving the multi-commodity flow instance corresponding to $(G, \Sigma)$ as per Remark 2.2.2.

Signed graph $(G, \Sigma)$ has no odd- $K_{5}$ minor if and only if $(M,\{e\})$ has no $\left(T_{11}^{*},\left\{e_{11}\right\}\right)$ minor. Minor operations in $(M,\{e\})$ can be shown to correspond to minor operations in $(G, \Sigma)$. Thus Theorem 2.4.2 proves Theorem 1.1.4 regarding Eulerian integer multicommodity flow for graphs and Theorem 2.4.3 proves Theorem 1.1.3 regarding fractional multi-commodity flow for graphs. 


\subsubsection{Even Cut Matroids}

Let $M=\operatorname{ecut}(G, T)$ for graph $G=(V, E)$ and $T \subseteq V$ with $|T|$ even. For $e=s t \in \Sigma$, $e$-paths of $M$ are the $s, t$-separating $T$-odd cuts of $G$. We say $\delta(S)$ as an $s, t$-separating $T$-odd cut if $s \in S, t \notin S$ and $|T \cap S|$ is odd. The $e$-paths of an even cut matroid are st-T-cuts. If $e$ is a pendent edge $(e=\delta(v)$ for some $v \in V)$, then the $e$-paths of $M$ are simply the $T$-cuts of $(G, T)$.

The single commodity Flowing Conjecture (Conjecture 2.2.4) is true for even cut matroids, but the single commodity Cycling Conjecture (Conjecture 2.2.5) remains open.

Theorem 2.4.4. If $M$ is an even cut matroid, then $(M,\{e\})$ has the fractional $M F M C$ property if and only if it has no $\left(T_{11},\left\{e_{11}\right\}\right)$ or $\left(A G(3,2),\left\{e_{8}\right\}\right)$ minor.

\subsubsection{Duals of Even Cycle Matroids}

Recall that if $M=\operatorname{ecycle}(G, \Sigma)$ for $G=(V, E)$ and $\Sigma \subseteq E$, then the elements of $M^{*}$ are cuts and signatures of $(G, \Sigma)$. The $e$-paths of $M^{*}$ are the $s$-bonds and signatures of $(G, \Sigma)$. The single commodity Flowing Conjecture (Conjecture 2.2.4) holds for duals of even cycle matroids (follows from [19] and relationship between fractional flow in matroids and their duals - see Theorem 3.1.1).

Theorem 2.4.5. If $M^{*}$ is the dual of even cycle matroid $M$, then $\left(M^{*},\{e\}\right)$ has the fractional MFMC property if and only it has no $\left(T_{11},\left\{e_{11}\right\}\right)$ or $\left(A G(3,2),\left\{e_{8}\right\}\right)$ minor.

The single commodity Cycling Conjecture (Conjecture 2.2.5) is still open for duals of even cycle matroids. However, it has been shown to imply the Four Colour Theorem: "every planar graph is 4-(vertex)-colourable".

Claim 2.4.6. The single commodity Cycling Conjecture (Conjecture 2.2.5) for duals of even cycle matroids implies the Four Colour Theorem.

Proof. Let $G=(V, E)$ be a simple plane graph, $H$ be obtained from $G$ by adding loop $e$, and $\Sigma=E(H)$. Let $M$ be the dual of ecycle $(H, \Sigma)$. First, we describe the $e$-paths and e-cuts of $M$.

The cycles of $M$ are signatures of $(H, \Sigma)$ or cuts of $H$. Since no cut contains a loop, the $e$-paths of $M$ are the signatures of $(G, E(G))$. That is, the $e$-paths of $M$ are exactly the odd circuit covers of $G$. 
Since the $e$-cuts of $M$ are the $e$-paths of $M^{*}$, the $e$-cuts of $M$ are of the form $D-e$ where $D$ is a cocyle of $M$ using $e$. In particular, $D$ is an even cycle of $M$. Since $e$ is an odd loop, the $e$-cuts of $M$ are exactly the odd cycles of $G$.

Consider the unit weight function. The Eulerian condition holds if $|D|$ is even for all cocycles of $M$. Since the cocycles of $M$ are the even cycles of $(H, \Sigma)$ and $\Sigma=E(H)$, the unit weight function is Eulerian.

If the single commodity Cycling Conjecture holds, and $(H, \Sigma)$ has no obstruction, then the size of a minimum length odd circuit is equal to the maximum number of disjoint signatures. Since $G$ is simple, the length of the shortest odd circuit is at least 3 and so there exists disjoint signatures $\Sigma_{1}$ and $\Sigma_{2}$.

Suppose $\Sigma_{1}$ is the complement of the cut $\delta(A)$ and $\Sigma_{2}$ is the complement of the cut $\delta(B)$. Partition the vertices into sets $V_{1}=A \cap B, V_{2}=A \cap \bar{B}, V_{3}=\bar{A} \cap B$ and $V_{4}=\bar{A} \cap \bar{B}$. If $V_{1}, V_{2}, V_{3}$ and $V_{4}$ are stable sets of $G, G$ is 4-colourable. By way of contradiction, suppose there exists edge $e=x y$ such that $x, y \in V_{i}$. Then $e \in \Sigma_{1}$ and $e \in \Sigma_{2}$, contradicting the fact $\Sigma_{1}$ and $\Sigma_{2}$ are disjoint. So $\left(V_{1}, V_{2}, V_{3}, V_{4}\right)$ give a 4-colouring of $V(G)$.

If $G$ is planar, it has no $K_{5}$ minor (Kuratowski's Theorem) and so $(H, \Sigma)$ has no obstruction. It follows that $G$ has a 4-colouring. Therefore, assuming the single commodity Cycling Conjecture for duals of even cycle matroids, one can prove the Four Colour Theorem.

\subsubsection{Duals of Even Cut Matroids}

Recall that if $M=\operatorname{ecut}(G, T)$ for $G=(V, E)$ and $T \subseteq V$, then the elements of $M^{*}$ are cycles of $G$ and $T$-joins of $(G, T)$. The $e$-paths of $M^{*}$ are the st-paths of $G$ and $T$-join of $(G, T)$. The single commodity Flowing Conjecture (Conjecture 2.2.4) holds for duals of even cut matroids (follows from [19] and relationship between fractional flow in matroids and their duals - see Theorem 3.1.1).

Theorem 2.4.7. If $M^{*}$ is the dual of even cut matroid $M$, then $\left(M^{*},\{e\}\right)$ has the fractional $M F M C$ property if and only if it has no $\left(T_{11}^{*},\left\{e_{11}\right\}\right)$ or $\left(A G(3,2),\left\{e_{8}\right\}\right)$ minor.

The single commodity Cycling Conjecture (Conjecture 2.2.5) is still open for duals of even cut matroids. However, it too has been shown to imply the Four Colour Theorem.

Claim 2.4.8. The single commodity Cycling Conjecture (Conjecture 2.2.5) for duals of even cut matroids implies the Four Colour Theorem. 
Proof. First note a result of Tait [32] shows that the Four Colour Theorem is equivalent to the statement: "Every simple 2-connected cubic planar graph $G$ is 3-edge colourable." Let $G$ be a simple 2-connected cubic graph. The unit weight function is Eulerian and so the Cycling Conjecture (Conjecture 2.2.5) implies that $G$ has 3 disjoint $T$-joins as long as $G$ is not contractible to the Petersen graph. Since all vertices have degree 3 , this is equivalent to being able to find 3 disjoint matchings. Assigning one colour class to each matching gives a 3-edge colouring of $G$. The Petersen graph is not planar, implying the Four Colour Theorem.

It was, in fact, a conjecture of Tutte [34] that every simple 2-connected cubic graph that is not 3-edge-colourable is contractible to the Petersen graph. This conjecture was resolved by Robertson, Sanders, Seymour and Thomas [27] [26]. Tutte's conjecture has been generalized by Conforti and Johnson [5]; the more general conjecture would also follow from the single commodity Cycling Conjecture (Conjecture 2.2.5) for duals of even cut matroids.

Conjecture 2.4.9 (Conforti Johnson Conjecture). In graphs not contractible to the Petersen graph, the number of pairwise disjoint postman sets is equal to the size of a minimum odd cut.

\subsubsection{A Note on the Restriction to Binary Matroids}

Although we have restricted ourselves to binary matroids, all the flow definitions can be applied to general matroids. It has been noted previously [29] that the fractional, integer and Eulerian integer flow problems in non-binary matroids are (if you are a pessimist) uninteresting or (if you are an optimist) already solved. The matroid $U_{4}^{2}$ has a 4 element ground set and every 2 element subset thereof is independent. For any element $f$, there is no fractional $w$-flow for instance $\left(U_{4}^{2}, f, w\right)$ where $w_{e}=1$ for all $e \in E-f$ and $w_{f}=2$. A result of Bixby [3] states that if $M$ is connected and non-binary, then $M$ has a $U_{4}^{2}$ minor using $f$. Thus single commodity flow instances for non-binary matroids can be disregarded.

\subsection{Relating Flows to Clutters}

\subsubsection{Definitions}

A clutter $\mathcal{F}$ on ground set $E$ is a finite family of sets of $E:=E(\mathcal{F})$ such that no set in $\mathcal{F}$ contains or is equal to some other set of $\mathcal{F}$. That is, there does not exist distinct 
$F_{1}, F_{2} \in \mathcal{F}$ such that $F_{1} \subseteq F_{2}$. A set $B \subseteq E$ is a cover of $\mathcal{F}$ if $B \cap S \neq \emptyset$ for all $S \in \mathcal{F}$. The set of all inclusion-wise minimal covers of $\mathcal{F}$ forms a clutter $b(\mathcal{F})$ called the blocker of $\mathcal{F}$. As $b(b(\mathcal{F}))=\mathcal{F}[6]$, we call a pair of clutters $(\mathcal{F}, \mathcal{K})$ a blocking pair if $\mathcal{K}=b(\mathcal{F})$. A clutter $\mathcal{F}$ is binary if for all $S_{1}, S_{2}, S_{3} \in \mathcal{F}$ there exists $S \in \mathcal{F}$ such that $S \subseteq S_{1} \triangle S_{2} \triangle S_{3}$. Equivalently, $\mathcal{F}$ is binary if for all $S \in \mathcal{F}$ and $T \in b(\mathcal{F}),|S \cap T|$ is odd [6].

The following results relate clutters and matroids and allow us to switch from discussing flows in matroids to discussing packings in clutters. Proofs appear in past work; see [19] for example.

Proposition 2.5.1. Let $M$ be a binary matroid and let $e \in E(M)$.

1. The set of e-paths of $M$ is a binary clutter.

2. The set of e-cuts of $M$ is a binary clutter.

3. The clutter of e-paths and e-cuts form a blocking pair.

Moreover, every binary clutter is the set of e-paths for some binary matroid $M$ and some $e \in E(M)$.

Remark 2.5.2. The set of e-cuts of binary matroid $M$ is exactly the set of e-paths of $M^{*}$.

\subsubsection{The MFMC Properties}

Given clutter $\mathcal{F}$ with ground set $E$ and $w \in \mathbb{Z}_{+}^{E}$, call $\mathcal{P} \subseteq \mathcal{F}$ a $w$-packing of elements of $\mathcal{F}$ if $|P \in \mathcal{P}: f \in P| \leq w_{f}$ for all $f \in E$. The size of $\mathcal{P}$ is simply $|\mathcal{P}|$. Consider the following primal-dual pair of linear programs.

$$
\begin{array}{rlr}
\min & \sum\left(w_{f} x_{f}: f \in E\right) & \\
\text { subject to } & \sum_{x \geq \mathbb{O}}\left(x_{f}: f \in S\right) \geq 1 & \\
\max & \sum\left(y_{S}: S \in \mathcal{F}\right) & \\
\text { subject to } & \sum\left(y_{S}: f \in S \in \mathcal{F}\right) \leq w_{f} & \text { for all } S \in \mathcal{F} \\
& & \\
& & \\
& &
\end{array}
$$

Observe that if $\mathcal{F}$ is the clutter of $e$-paths for binary matroid $M$ and $e \in E(M)$, then (2.5) is the same as (2.3) and (2.6) is the same as (2.4). The restriction of (2.5) to the integers 
models the problem of finding the minimum weight cover of $\mathcal{F}$ (a minimum weight element of $b(\mathcal{F})$ ). The restriction of $(2.6)$ to the integers models the problem of finding maximum weight $w$-packing of elements of $\mathcal{F}$. In correspondence with our definitions for single commodity flow, we say that $\mathcal{F}$ has the

1. fractional MFMC property if for every $w \in \mathbb{Z}_{+}^{E}$ there is an integer solution to (2.5) and a solution to (2.6) with the same objective value,

2. integer $M F M C$ property if for every $w \in \mathbb{Z}_{+}^{E}$ there is an integer solution to (2.5) and an integer solution to (2.6) with the same objective value,

3. Eulerian integer MFMC property if for every Eulerian $w \in \mathbb{Z}_{+}^{E}$ there is an integer solution to (2.5) and an integer solution to (2.6) with the same objective value.

By equivalence of definition: $(M,\{e\})$ has the fractional MFMC property if and only if the clutter of $e$-paths of $M$ has the fractional MFMC property; $(M,\{e\})$ has the integer MFMC property if and only if the clutter of $e$-paths of $M$ has the integer MFMC property; and $(M,\{e\})$ has the Eulerian integer MFMC property if and only if the clutter of $e$-paths of $M$ has the Eulerian integer MFMC property. That is, instead of looking for single commodity $w$-flows, we can concern ourself with $w$-packings of clutters of $e$-paths.

\subsubsection{Flowing and Cycling Conjectures for Clutters}

Given clutter $\mathcal{F}$ and $f \in E(\mathcal{F})$, we define $\mathcal{F} \backslash f$ as $\{S \in \mathcal{F}: f \notin S\}$ and say $\mathcal{F} \backslash f$ is obtained from $\mathcal{F}$ by deleting $f$. Given clutter $\mathcal{F}$ and $f \in E(\mathcal{F})$, we define $\mathcal{F} / f$ as the set of inclusion-wise minimal sets of $\{S-f: S \in \mathcal{F}\}$ and say $\mathcal{F} / f$ is obtained from $\mathcal{F}$ by contracting $f$. A minor of $\mathcal{F}$ is any clutter $\mathcal{F}^{\prime}$ obtained by a sequence of deletions and contractions. As deletions and contractions associate, we can unambiguously write $\mathcal{F}^{\prime}=\mathcal{F} / I \backslash J$ to indicate that the elements of $I \subseteq E(\mathcal{F})$ were deleted and the elements of $J \subseteq E(\mathcal{F})$ were contracted to obtain $\mathcal{F}^{\prime}$ from $\mathcal{F}$. It is straightforward to verify that any minor of a binary clutter is binary. Moreover, it is straightforward to show that if $\mathcal{F}$ is the clutter of $e$-paths of $M$, then $\mathcal{F} \backslash f$ is the clutter of $e$-paths of $M \backslash f$ and $\mathcal{F} / f$ is the clutter of $e$-paths of $M / f$.

The clutter $\mathcal{Q}_{6}$ is the clutter of odd circuits of $K_{4}$, the clutter $\mathcal{O}_{K_{5}}$ is the clutter of odd circuits of $K_{5}$, and the clutter $\mathcal{L}_{7}$ is the clutter of lines of the Fano plane. Two clutters are isomorphic to each other if one can be obtained from the other by relabelling elements of the ground set. Seymour's Theorem (Theorem 1.2.2) and the Flowing and Cycling Conjectures (Conjectures 1.2.3 and 1.2.4) can be restated as follows. 
Theorem 2.5.3 (Seymour). A binary clutter has the integer MFMC property if and only if it does not have a minor isomorphic to

$$
\mathcal{Q}_{6}=\{\{1,2,4\},\{1,3,5\},\{2,3,6\},\{4,5,6\}\} .
$$

Conjecture 2.5.4 (Flowing Conjecture). A binary clutter is has the fractional MFMC property if and only if it does not have any of the following clutters as a minor: $\mathcal{O}_{K_{5}}$, $b\left(\mathcal{O}_{K_{5}}\right)$, or $\mathcal{L}_{7}$.

Conjecture 2.5.5 (Cycling Conjecture). A binary clutter has the Eulerian MFMC property if and only if it does not have any of the following clutters as a minor: $\mathcal{O}_{K_{5}}, b\left(\mathcal{O}_{K_{5}}\right)$, $\mathcal{L}_{7}$, or $\mathcal{P}_{T}$. The clutter $\mathcal{P}_{T}$ corresponds to the postman sets of the Petersen graph.

\subsubsection{Flowing and Cycling Results for Clutters}

The following chart summarizes the implications of Section 2.4 to clutters.

\begin{tabular}{|c|c|c|c|}
\hline & Integer & Fractional & Eulerian Integer \\
\hline General & $\mathcal{Q}_{6}$ & Flowing Conjecture & Cycling Conjecture \\
\hline st-Paths & - & - & - \\
\hline st-Cuts & - & - & - \\
\hline Odd Circuits & $\mathcal{Q}_{6}$ & $\mathcal{O}_{K_{5}}$ & $\mathcal{O}_{K_{5}}$ \\
\hline Odd-st-Walks & $\mathcal{Q}_{6}$ & $\mathcal{O}_{K_{5}}, \mathcal{L}_{7}$ & $\begin{array}{l}\text { Holds for non-rooted } \\
\text { case [1] }\end{array}$ \\
\hline st-T-cuts & $\mathcal{Q}_{6}$ & $b\left(\mathcal{O}_{K_{5}}\right), \mathcal{L}_{7}$ & Open \\
\hline $\begin{array}{l}\text { Blocker of } \\
\text { Odd-st-Walks }\end{array}$ & $\mathcal{Q}_{6}$ & $b\left(\mathcal{O}_{K_{5}}\right), \mathcal{L}_{7}$ & $\begin{array}{l}\text { Implies 4-Colour The- } \\
\text { orem }\end{array}$ \\
\hline $\begin{array}{l}\text { Blocker of st- } \\
T \text {-cuts }\end{array}$ & $\mathcal{Q}_{6}$ & $\mathcal{O}_{K_{5}}, \mathcal{L}_{7}$ & $\begin{array}{l}\text { Implies Conforti John- } \\
\text { son Conjecture }\end{array}$ \\
\hline
\end{tabular}

Table 2.1: Whenever a minor or minors are listed, this indicates these minors are known to be the obstructions to the affiliated MFMC property. An entry of "-" implies there are no obstructions. Otherwise, the entry gives information about the related conjecture. 


\section{Chapter 3}

\section{A Constructive Version of Lehman's Theorem}

\subsection{Preliminaries}

\subsubsection{Set Covering Polyhedra, Idealness, and Lehman's Theorem}

Given a 0,1-matrix $M$ we define the affiliated set covering polyhedron by

$$
Q(M):=\{x \geq \mathbb{O}: M x \geq \mathbb{1}\}
$$

We say $M$ is ideal if $Q(M)$ is integral; i.e., if every extreme point of $Q(M)$ is integral. Given a clutter $\mathcal{F}$, let $M(\mathcal{F})$ denote the 0,1 -matrix with columns indexed by elements $f \in E(M)$ and rows indexed by sets $F \in \mathcal{F}$, where entry $(F, f)$ is 1 if and only if $f \in F$. Observe that $M(\mathcal{F})$ is only defined up to permutations of the rows. We say clutter $\mathcal{F}$ is ideal if $M(\mathcal{F})$ is ideal, and write $Q(\mathcal{F})$ for $Q(M(\mathcal{F}))$. Note that if there exists $w \in \mathbb{Z}_{+}^{E}$ such that $\min \left\{w^{T} x: x \in Q(\mathcal{F})\right\}$ is fractional, then $\mathcal{F}$ is not ideal. Ideal clutters are exactly those clutters for which the fractional MFMC property holds. A theorem of Lehman [22] relates idealness in a clutter to idealness in its blocker.

Theorem 3.1.1. A clutter is ideal if and only if its blocker is.

Note that we have already explicitly and implicitly used this theorem. For example, it shows that $(M,\{e\})$ has the fractional MFMC property if and only if $\left(M^{*},\{e\}\right)$ has the fractional MFMC property. 
We call clutter $\mathcal{F}$ minimally nonideal (mni) if it is not ideal but all its proper minors are ideal. An example of a mni clutter is the clutter whose ground set and elements correspond, respectively, to the points and lines of the finite degenerate projective plane. This clutter on ground set $\{0,1 \ldots s\}$ is given by

$$
\mathcal{J}_{s}=\{\{1 \ldots s\},\{0,1\},\{0,2\}, \ldots,\{0, s\}\}
$$

for $s \geq 2$.

A theorem of Lehman [23] gives a characterization of mni clutters and shows, surprisingly, that set covering polyhedra corresponding to minimally nonideal clutters have exactly one fractional extreme point.

Theorem 3.1.2 (Lehman's Theorem). Let $\mathcal{F}$ be a minimally non-ideal clutter that is not isomorphic to $\mathcal{J}_{s}$ for $s \geq 2$. Let $\mathcal{K}$ be the blocker of $\mathcal{F}$. Denote by $\overline{\mathcal{F}}$ the clutter formed by the sets of minimum cardinality of $\mathcal{F}$. Denote by $\overline{\mathcal{K}}$ the clutter formed by the sets of minimum cardinality of $\mathcal{K}$. Then

(L1) $M(\overline{\mathcal{F}})$ and $M(\overline{\mathcal{K}})$ are square matrices, and

(L2) after possibly rearranging rows of $M(\overline{\mathcal{F}})$ we have for some $d \geq 1$

$$
M(\overline{\mathcal{F}}) M(\overline{\mathcal{K}})^{T}=J+d I .
$$

where $J$ is the dimension $n$ square matrix of $1 s$ and $I$ is the dimension $n$ identity matrix.

In the preceding theorem, if $r$ denotes the cardinality of the sets of $\overline{\mathcal{F}}$, then $\frac{1}{r} \mathbb{1}$ is the unique fractional extreme point of $Q(\mathcal{F})$. If $\ell$ denotes the cardinality of the sets of $\overline{\mathcal{K}}$, then $\frac{1}{\ell} \mathbb{1}$ is the unique fractional extreme point of $Q(\mathcal{K})$. The clutters $\mathcal{O}_{K_{5}}, b\left(\mathcal{O}_{K_{5}}\right)$ and $\mathcal{L}_{7}$ are all minimally nonideal. In Section 3.4, an interpretation of equation (3.1) is given for clutter $\mathcal{O}_{K_{5}}$.

Let $(\mathcal{F}, \mathcal{K})$ be a blocking pair, $\overline{\mathcal{F}}$ denote the clutter formed by the minimum cardinality sets of $\mathcal{F}$, and $\overline{\mathcal{K}}$ denote the clutter formed by the minimum cardinality sets of $\mathcal{K}$. We say that $\mathcal{F}$ is a Lehman clutter whenever conditions (L1) and (L2) of Lehman's Theorem are satisfied. Note that if $\mathcal{F}$ is a Lehman clutter, so also is its blocker $\mathcal{K}$. Hence we refer to $(\mathcal{F}, \mathcal{K})$ as a Lehman blocking pair.

\subsubsection{Statement of Constructive Version}

Consider the decision problem: is a given clutter $\mathcal{F}$ ideal? It is clearly in Co-NP as it suffices to exhibit a fractional extreme point of $Q(\mathcal{F})$. Outcomes (1) and $(2)$ of the previous 
theorem are also a Co-NP certificate. For any nonideal clutter $\mathcal{F}$, there exists a clutter minor of $\mathcal{F}$ that is a Lehman clutter. If $\mathcal{F}^{\prime}=\mathcal{F} / I \backslash J$, then $I, J, M\left(\mathcal{F}^{\prime}\right)$ and $M\left(b\left(\mathcal{F}^{\prime}\right)\right)$ also give a certificate that $\mathcal{F}$ is nonideal. We are interested in finding such certificates efficiently. Firstly, doing so allows us to construct a highly regular Co-NP certificate of nonidealness from an arbitrary fractional extreme point. Moreover, combining this with existing excluded minor results allows us to find excluded minors for idealness.

Before stating the main theorem of this section, we note that the result relies upon the ellipsoid method. As is typical in algorithms using the ellipsoid method, the algorithm's runtime is analyzed with respect to the number of calls to a separation oracle. Let $P \subseteq \mathbb{R}^{n}$ be a polyhedron. The separation problem for $P$ and a point $\bar{x} \in \mathbb{Q}^{n}$ is to either determine that $\bar{x} \in P$, or to find a separating constraint $a^{T} x \leq \beta$ (i.e., $a^{T} \bar{x}>\beta$ and $a^{T} x \leq \beta$ for all $x \in P)$. A separation oracle for $P$ is a function that solves the separation problem for any $\bar{x} \in \mathbb{Q}^{n}$. We are now ready to state our algorithmic version of Theorem 3.1.2.

Theorem 3.1.3. Let $\mathcal{F}$ be a clutter and suppose that $Q(\mathcal{F}) \subseteq \mathbb{R}^{n}$ is given by a separation oracle. Let $x$ be a fractional extreme point of $Q(\mathcal{F})$ and suppose that we are given $n$ facets of $Q(\mathcal{F})$ that define $x$. Then in oracle polynomial time of $n$ we can find disjoint sets $I, J \subseteq E(\mathcal{F})$ such that for $\mathcal{F}^{\prime}=\mathcal{F} / I \backslash J$ either

1. $\mathcal{F}^{\prime}$ is isomorphic to $\mathcal{J}_{s}$, or

2. $\mathcal{F}^{\prime}$ is a Lehman clutter.

Moreover, in case (2) we also find all the minimum cardinality sets of $\mathcal{F}^{\prime}$.

\subsection{Proof Outline}

\subsubsection{Key Definitions and Lemmas}

Throughout this section, $\mathcal{F}$ will always denote a clutter with $E(\mathcal{F})=[n]^{1}$. Given a clutter $\mathcal{F}$, we denote by $P(\mathcal{F})$ the polytope $Q(\mathcal{F}) \cap[0,1]^{n}$.

Proposition 3.2.1. Polyhedron $P(\mathcal{F})$ is integral if and only if $Q(\mathcal{F})$ is integral.

Proof. Suppose $P(\mathcal{F})$ is integral and $\bar{x}$ is a fractional extreme point of $Q(\mathcal{F})$.

First consider the case when $\bar{x} \in P(\mathcal{F})$. Since $\bar{x}$ cannot be a fractional extreme point of $P(\mathcal{F})$, we can write $\bar{x}=\frac{1}{2} x^{1}+\frac{1}{2} x^{2}$ for $x^{1}, x^{2} \in P(\mathcal{F})$. Since $P(\mathcal{F}) \subset Q(\mathcal{F}), x^{1}, x^{2} \in Q(\mathcal{F})$; this contradicts the fact $\bar{x}$ is an extreme point of $Q(\mathcal{F})$.

\footnotetext{
${ }^{1}$ Here $[n]$ denotes the set $\{1,2 \ldots n\}$.
} 
Now, consider the case when $\bar{x} \notin P(\mathcal{F})$. There exists $i \in[n]$ such that $\bar{x}_{i}>1$. Define $x^{1}$ by $x_{j}^{1}=\bar{x}_{j}$ for all $j \neq i$ and $x_{i}^{1}=\bar{x}_{i}-\epsilon$. Define $x^{2}$ by $x_{j}^{2}=\bar{x}_{j}$ for all $j \neq i$ and $x_{i}^{2}=\bar{x}_{i}+\epsilon$. Choosing $\epsilon$ small enough, $x^{1}, x^{2} \in Q(\mathcal{F})$. Then $\frac{1}{2} x^{1}+\frac{1}{2} x^{2}=\bar{x}$, contradicting the fact $\bar{x}$ is an extreme point of $Q(\mathcal{F})$.

Suppose $Q(\mathcal{F})$ is integral and $\bar{x}$ is a fractional extreme point of $P(\mathcal{F})$.

If $x_{i}<1$ for all $i$, then $\bar{x}$ is also a fractional extreme point of $Q(\mathcal{F})$. Otherwise we can write $\bar{x}=\frac{1}{2} x^{1}+\frac{1}{2} x^{2}$ for $x^{1}=\bar{x}+\epsilon d$ and $x^{2}=\bar{x}-\epsilon d$. Choosing $\epsilon$ small enough, $x^{1}, x^{2} \in P(\mathcal{F})$, contradicting the fact $\bar{x}$ is an extreme point of $P(\mathcal{F})$. However, concluding $\bar{x}$ is is a fractional extreme point of $Q(\mathcal{F})$ contradicts the fact $Q(\mathcal{F})$ is integral. Thus there exists $i \in[n]$ such that $i=1$.

Project $P(\mathcal{F})$ and $Q(\mathcal{F})$ onto $\left\{x: x_{i}=1\right\}$; that is, proceed inductively on $P(\mathcal{F} / i)$ and $Q(\mathcal{F} / i)$. Whenever $\mathcal{F}$ is a one element clutter, $P(\mathcal{F})$ is integral if and only if $Q(\mathcal{F})$ integral. It follows by induction that $P(\mathcal{F})$ is integral if and only if $Q(\mathcal{F})$ is integral.

Given a point $\bar{x} \in P(\mathcal{F})$ and $j \in[n]$, let $\bar{x}^{j}=\left(\bar{x}_{1}, \ldots, \bar{x}_{j-1}, 1, \bar{x}_{j+1}, \ldots, \bar{x}_{n}\right)^{T}$ and $F^{j}=P(\mathcal{F}) \cap\left\{x: x_{j}=1\right\}$. Note that $\bar{x}^{j} \in F^{j}$. Call point $\bar{x} \in P(\mathcal{F})$ special if it is an extreme point and for all $j \in[n]$

(S1) $0<\bar{x}_{j}<1$,

(S2) $\bar{x}^{j}$ can be expressed as a convex combination of integer extreme points of $F^{j}$.

Our algorithm relies on the following lemma.

Lemma 3.2.2. Suppose $P(\mathcal{F})$ is given by a separation oracle. Given an extreme point $\bar{x}$ of $P(\mathcal{F})$ with $n$ facets that define $\bar{x}$, we can, in oracle polynomial time in $n$, either

1. deduce that $\bar{x}$ is special, or

2. find $j \in[n]$ and a fractional extreme point $x^{\prime}$ of $P(\mathcal{F} / j)$, or

3. find $j \in[n]$ and a fractional extreme point $x^{\prime}$ of $P(\mathcal{F} \backslash j)$.

Moreover, for outcomes (2) and (3) we also find $n-1$ facets that define $x^{\prime}$.

Two extreme points in a polytope are adjacent if they are contained in a face of dimension 1 . The set of all extreme points that are adjacent to extreme point $\bar{x}$ are the neighbours of $\bar{x}$. Extreme point $\bar{x}$ of polyhedron $P \subseteq \mathbb{R}^{n}$ is non-degenerate if there are exactly $n$ facets of $P$ satisfied at equality at $\bar{x}$ or, equivalently, if $\bar{x}$ has exactly $n$ neighbours in $P$. These definitions are sufficient to state the next set of required lemmas.

Lemma 3.2.3. Special points of $P(\mathcal{F})$ are non-degenerate. 
Lemma 3.2.4. Suppose we are given a non-degenerate extreme point $\bar{x}$ of $P(\mathcal{F})$ where $0<\bar{x}_{j}<1$ for all $j \in[n]$, and $n$ facets that define $\bar{x}$. If $P(\mathcal{F})$ is described by a membership oracle, then we can, in oracle polynomial time of $n$, find the $n$ neighbours of $\bar{x}$.

Lemma 3.2.5. No two special points of $P(\mathcal{F})$ are adjacent.

Lemma 3.2.6. Suppose that $\bar{x}$ is a special point of $P(\mathcal{F})$ and that all neighbours of $\bar{x}$ are integral. The facets that define $\bar{x}$ are of the form $\sum_{k \in S_{i}} x_{k} \geq 1$ where $S_{i} \in \mathcal{F}$ for $i=1, \ldots, n$. Let $\overline{\mathcal{F}}=\left\{S_{1}, \ldots, S_{n}\right\}$; either

1. $\mathcal{F}$ is isomorphic to $\mathcal{J}_{s}$ and $\overline{\mathcal{F}}=\mathcal{F}$, or

2. $\mathcal{F}$ is a Lehman clutter and the elements of $\overline{\mathcal{F}}$ are the minimum cardinality sets of $\mathcal{F}$.

Note that in Lemma 3.2.6, $\bar{x}$ is necessarily non-degenerate because of Lemma 3.2.3.

\subsubsection{Algorithm}

Let $\bar{x}$ be an extreme point of $P(\mathcal{F})$ and suppose that we are given $n$ facets of $P(\mathcal{F})$ that define $\bar{x}$. We apply the algorithm referenced in Lemma 3.2.2. If outcome (2) or (3) occurs, then we apply the main algorithm recursively to $P(\mathcal{F} / i)$ or $P(\mathcal{F} \backslash i)$ respectively, with the new extreme point $x^{\prime}$ and the $n-1$ facets defining $x^{\prime}$. Note that the separation oracle for $P(\mathcal{F})$ extends to a separation oracle for $P(\mathcal{F} / j)$ and $P(\mathcal{F} \backslash j)$, as contracting $j$ corresponds to setting $\bar{x}_{j}=0$ and deleting $j$ to setting $\bar{x}_{j}=1$.

Otherwise, outcome (1) of Lemma 3.2.2 occurs. Because of Lemma 3.2.3, $\bar{x}$ is nondegenerate. Using the algorithm referenced in Lemma 3.2.4 we can find its neighbours $b^{1}, \ldots, b^{n}$. Consider first the case where all of $b^{1}, \ldots, b^{n}$ are integer. Since we have the facets that define $\bar{x}$, we can construct $\overline{\mathcal{F}}$ as in Lemma 3.2.6. Then outcome (1) and (2) of Lemma 3.2.6 correspond respectively to outcomes (1) and (2) of Theorem 3.1.3 and we can stop.

Thus we may assume that $b^{i}$ is fractional for some $i \in[n]$. We apply the algorithm referenced in Lemma 3.2.2 to $b^{i}$. As $b^{i}$ is not special (see Lemma 3.2.5), outcome (2) or (3) occurs, and we can again apply the main algorithm recursively.

It is straightforward to verify that the algorithm runs in oracle polynomial time. Since $Q(\mathcal{F})$ is described by a separation oracle then, so is $P(\mathcal{F})$ as it suffices to check in addition that $x_{e} \leq 1$ for all $e \in E(\mathcal{F})$. Note that this algorithm may call itself recursively. Between recursive calls, we use the polynomial time algorithm referenced in Lemma 3.2.2 at most twice and the polynomial time algorithm referenced in Lemma 3.2.4 once. The polynomiality follows because every time we call the algorithm recursively we decrease the dimension of the polytope in consideration by one and so at most $n$ recursive calls are made. 


\subsection{Technical Sections of Proof}

\subsubsection{Polyhedral Proofs}

The following polyhedral theory definitions are found in Grötschel et. al. (see [16] Chapter 6.2 . Let $P \subseteq \mathbb{R}^{n}$ be a polyhedron and let $\phi$ be positive integer. We say that $P$ has facet-complexity at most $\phi$ if there exists a system of inequalities with rational coefficients that has solution set $P$ where the encoding length of each inequality in the system is at most $\phi$. (If $P \subseteq \mathbb{R}^{n}$, we require $\phi \geq n+1$.) A well-described polyhedron is a triple $(P ; n, \phi)$ where $P \subseteq \mathbb{R}^{n}$ is a polyhedron with facet-complexity at most $\phi$. The encoding length $\langle P\rangle$ of well-described polyhedron $(P ; n, \phi)$ is at most $\phi+n$.

The proof of Lemma 3.2.2 requires the following algorithmic version of Caratheodory's Theorem (see [16] Theorem 6.5.11).

Proposition 3.3.1. Let $P \subseteq \mathbb{R}^{n}$ be a well-described polytope given by a separation oracle and let $\bar{x} \in P \cap \mathbb{Q}^{n}$. There exists an oracle-polynomial algorithm that will express $\bar{x}$ as a convex combination of at most $\operatorname{dim}(P)+1$ extreme points of $P$. Moreover, for each of these points we can find $n$ defining facets of $P$.

We will apply this proposition to show that given a separation oracle for $P(\mathcal{F})$, we can express any point $\bar{x} \in P(\mathcal{F}) \cap \mathbb{Q}^{n}$ as a convex combination of extreme points of $P(\mathcal{F})$. To do this, we show that $P(\mathcal{F})$ is indeed well-described. Since $P(\mathcal{F})$ can be described by a system of inequalities with 0,1 coefficients, the facet-complexity of $P(\mathcal{F})$ is at most $n+1$ and so $(P(\mathcal{F}) ; n+1, n)$ is a well-described polyhedron.

Corollary 3.3.2. Let $P(\mathcal{F})$ be given by a separation oracle and let $\bar{x} \in P(\mathcal{F}) \cap \mathbb{Q}^{n}$. There exists an oracle-polynomial time algorithm that will express $\bar{x}$ as a convex combination of at most $\operatorname{dim}(P)+1$ extreme points of $P(\mathcal{F})$. Moreover, for each of these points, we can find $n$ defining facets of $P(\mathcal{F})$.

Using this corollary, we are ready to prove Lemma 3.2.2.

Proof of Lemma 3.2.2. Suppose $\bar{x}_{j} \in\{0,1\}$ for some $j \in[n]$. Let $x^{\prime}=\left(\bar{x}_{1}, \ldots, \bar{x}_{j-1}\right.$, $\left.\bar{x}_{j+1}, \ldots, \bar{x}_{n}\right)^{T}$. If $\bar{x}_{j}=0$ then $x^{\prime}$ is an extreme point of $P(\mathcal{F} / j)$ and the facets that define $\bar{x}$, omitting $x_{j}=0$, define $x^{\prime}$ and outcome (2) of the lemma occurs. If $\bar{x}_{j}=1$ then $x^{\prime}$ is an extreme point of $P(\mathcal{F} \backslash j)$ and the facets that define $\bar{x}$, omitting $x_{j}=1$, define $x^{\prime}$ and outcome (3) of the lemma occurs. Thus we may assume condition (S1) holds. 
For every $j \in[n]$, use Proposition 3.3.2 to express $\bar{x}^{j}$ as a convex combination of extreme points $y^{1}, \ldots, y^{k}$ of $F^{j}$ where $k \leq n$. Note, that as $\bar{x}$ is an extreme point of $P(\mathcal{F})$, it has encoding length $\langle\bar{x}\rangle$ polynomial in $n$ (see [16] Theorem 6.2.4). Suppose $y^{s}$ is fractional for some $s \in[k]$, then $\left(y_{1}^{s}, \ldots, y_{j-1}^{s}, y_{j+1}^{s}, \ldots, y_{n}^{s}\right)^{T}$ is an extreme point $P(\mathcal{F} \backslash j)$ and outcome (3) occurs. If this never occurs, condition (S2) holds and $\bar{x}$ is special. Note that even if outcome (2) or (3) occurs, this does not imply $\bar{x}$ is not special (only that we have not succeeded in demonstrating that it is special).

No additional definitions are needed to prove Lemma 3.2.4. We give a sketch of the proof here; a true proof would require introducing more machinery (Cramer's rule to bound denominators, for example).

Proof Sketch of Lemma 3.2.4. As $\bar{x}$ is non-degenerate, exactly $n$ constraints of $M(\mathcal{F}) \bar{x} \geq \mathbb{1}$ are tight for $\bar{x}$. Find $d \neq \mathbb{O}$ satisfying $n-1$ of these constraints. Then a neighbour $b$ of $\bar{x}$ is on the line $L=\{\bar{x}+\lambda d: \lambda \in \mathbb{R}\}$. Use the membership oracle to do a binary search to find $b \in L$.

\subsubsection{Proofs using Property $(\mathrm{P})$}

The remainder of the proofs follow closely the proof of Lehman's Theorem (Theorem 3.1.2) appearing in [6]. The following definition will be key: An $n \times n, 0,1$-matrix $M$ has property $(P)$ if

(P1) $M$ has no dominated row; that is, there do not exist rows $R_{i},{ }_{j}$ such that $\operatorname{supp}\left(R_{i}\right) \subseteq$ $\operatorname{supp}\left(R_{j}\right)$

(P2) $M$ has no column of $1 \mathrm{~s}$,

(P3) for all $i, j \in[n]$ where $M_{i, j}=0$,

$$
\sum_{k=1}^{n} M_{i k}=\sum_{k=1}^{n} M_{j k} .
$$

One key observation regarding matrices with property $(\mathrm{P})$ is that they cannot differ in exactly one row. The argument we give for this fact essentially appears in [6] Lemma 4.11.

Lemma 3.3.3. No pair of matrices with property $(P)$ differ in exactly one row.

Proof. Suppose for a contradiction that we have $n \times n$ matrices $M, M^{\prime}$ with property (P) where $M$ has rows $R_{1}, \ldots, R_{n}$ and $M^{\prime}$ has rows $R_{2}, \ldots, R_{n}, R_{n+1}$. Property (P1) shows 
there exists $j \in[n]$ such that entry $j$ of $R_{1}$ is 0 and entry $j$ of $R_{n+1}$ is 1 . Property (P2) states that column $j$ of $M^{\prime}$ is not a column of $1 \mathrm{~s}$, and so for some $i, 2 \leq i \leq n$, entry $j$ of $R_{i}$ is 0 . By property (P3), the number of $1 \mathrm{~s}$ in $R_{i}$ is equal to both the number of $1 \mathrm{~s}$ in column $j$ of $M$ and $M^{\prime}$. This is a contradiction as column $j$ of $M^{\prime}$ has one more 1 than column $j$ of $M$.

Let $\bar{x}$ be an extreme point of $P(\mathcal{F})$ where $0<\bar{x}_{j}<1$ for all $j \in[n]$. We say that $\overline{\mathcal{F}} \subseteq \mathcal{F}$ is a core of $\bar{x}$ if $M(\overline{\mathcal{F}}) \bar{x}=\mathbb{1}$, and $M(\overline{\mathcal{F}})$ is square and non-singular. Clearly, if $\bar{x}$ is an extreme point then there exists a core for $\bar{x}$. Moreover, the core is unique if and only if $\bar{x}$ is non-degenerate. We aim to show $M(\overline{\mathcal{F}})$ has property $(\mathrm{P})$ whenever $\overline{\mathcal{F}}$ is a core of a special point. We first require the following lemma due to de Bruijn and Erdös [8].

Lemma 3.3.4. Let $(I \cup J, E)$ be a bipartite graph with no isolated node. If $|I| \geq|J|$ and $d(i) \geq d(j)$ for all $i \in I, j \in J$ such that $i j \in E$, then $|I|=|J|$ and $d(i)=d(j)$ for all $i \in I, j \in J$ such that $i j \in E$.

Proof. Applying the given inequality and rearranging the summation gives

$$
|I|=\sum_{i \in I}\left(\sum_{j \in N(i)} \frac{1}{\operatorname{deg}_{G}(i)}\right) \leq \sum_{i \in I}\left(\sum_{j \in N(i)} \frac{1}{\operatorname{deg}_{G}(j)}\right)=\sum_{j \in J}\left(\sum_{i \in N(j)} \frac{1}{\operatorname{deg}_{G}(j)}\right)=|J|
$$

Since $|I| \geq|J|$, equality holds throughout and thus $|I|=|J|$ and $d(i)=d(j)$ for all $i \in I$, $j \in J$ such that $i j \in E$.

Lemma 3.3.5. Let $\bar{x}$ be a special point of $P(\mathcal{F})$ and let $\overline{\mathcal{F}}$ be a core of $\bar{x}$. Then $M(\overline{\mathcal{F}})$ has property $(P)$.

Proof. Matrix $M(\overline{\mathcal{F}})$ clearly satisfies $(\mathrm{P} 1)$ as $\mathcal{F}$ is a clutter. Matrix $M(\overline{\mathcal{F}})$ has property $(\mathrm{P} 2)$ as $M(\overline{\mathcal{F}})$ is nonsingular because $\overline{\mathcal{F}}$ is a core of $\bar{x}$ with special property (S1). Thus it suffices to show that $(\mathrm{P} 3)$ holds for $M(\overline{\mathcal{F}})$. The proof is nearly identical to that of Lemma 4.10 in [6] for minimally nonideal clutters. The property of being special ensures $M(\overline{\mathcal{F}})$ has the same necessary properties implied by the assumption $\mathcal{F}$ is minimally nonideal.

Consider $x^{j}$ defined previously by $x_{j}^{j}=1$ and $x_{k}^{j}=x_{k}$ for $k \neq j$. Clearly $x^{j} \in P(\mathcal{F})$ since $P(\mathcal{F})=\left\{x \in[0,1]^{n}: M(\mathcal{F}) \geq \mathbb{1}\right\}$. Let $F_{j}$ (not necessarily equal to $F^{j}$ defined previously) be the lowest dimensional face of $P(\mathcal{F}) \cap\left\{x: x_{j}=1\right\}$ that contains $x^{j}$. Unlike in the minimally nonideal case, $F_{j}$ is not necessarily integral. However, the result still follows by bounding the dimension of $F_{j}$ two different ways. 
Approach 1: The point $x^{j}$ lies at the intersection of the hyperplanes $\left\{x: x_{j}=1\right\}$ and $\left\{x: \overline{\left(a^{k}\right)^{T} x=1}\right\}$ for all $a^{k}$ rows of $M(\overline{\mathcal{F}})$ such that $a_{k j}=0$ There are at least $n-\sum_{k=1}^{n} a_{k j}$ equations defining hyperplanes of the second type and the hyperplane of the first type is independent of these. Thus

$$
\operatorname{dim}\left(F_{j}\right) \leq n-\left(n-\sum_{k=1}^{n} a_{k j}+1\right)=\sum_{k=1}^{n} a_{k j}-1 .
$$

Approach 2: Suppose that $\left(a^{i}\right)^{T}$ is such that $a_{i j}=0$. Since $\bar{x}$ is special, there exist integral $b^{1} \ldots b^{l}$ for $l \leq n$ such that $x^{j}=\sum_{t=1}^{l} \gamma_{t} b^{t}$ where $\gamma \geq 0, \mathbb{1}^{T} \gamma=1$. Since each $b^{t}$ is a $\{0,1\}$ vector

$$
\begin{array}{rlrl}
1 & =\left(a^{i}\right)^{T} x^{i} & & \text { since } a_{i j}=0 \text { and so such a constraint is also tight for } x^{j} \\
& =\sum_{t=1}^{l} \gamma_{l}\left(a^{i}\right)^{T} b^{t} & & \text { substituting for } x^{j} \\
& \geq 1 & \text { since }\left(a^{i}\right)^{T} b^{t} \geq 1 \text { as } b^{t} \in P(\mathcal{F}) .
\end{array}
$$

So, equality holds throughout. This implies that $\left(a^{i}\right)^{T} b^{t}=1$ for all $t \in\{1 \ldots l\}$ and thus $b^{t}$ has exactly one nonzero entry in every column $k$ such that $a_{j k}=1$. Furthermore, because $x_{k}^{j}=\sum_{t=1}^{l} \gamma_{t} b_{k}^{t}$ for all $k$ such that $a_{i k}=1$ and $x_{k}^{j}>0$ for all $k, F_{j}$ contains at least $\sum_{k=1}^{n} a_{i k}$ linearly independent points. Therefore,

$$
\operatorname{dim}\left(F_{j}\right) \geq \sum_{k=1}^{n} a_{i k}-1 .
$$

Combining bounds (3.2) and (3.3) regarding $\operatorname{dim}\left(F_{j}\right)$, we conclude that $\sum_{k=1}^{n} a_{i k} \leq$ $\sum_{k=1}^{n} a_{k j}$ for all $i, j \in[n]$ such that $a_{i j}=0$. Consider the bipartite representation of $J-$ $M(\overline{\mathcal{F}})$ given by graph $G=(V, E)$ with bipartition $(R, C)$ where $R=\{1, \ldots n\}$ corresponds to the rows of $M(\overline{\mathcal{F}})$ and $C=\left\{1^{\prime}, \ldots n^{\prime}\right\}$ corresponds to the columns of $M(\overline{\mathcal{F}})$. For $i \in$ $\{1 \ldots n\}$ and $j \in\left\{1^{\prime}, \ldots n^{\prime}\right\}, i j \in E$ if and only if $(J-A)_{i j}=1$. For $i \in\{1, \ldots n\}$, the degree of $i$ is $\operatorname{deg}_{G}(i)=n-\sum_{k=1}^{n} a_{i k}$. For $j \in\left\{1^{\prime} \ldots n^{\prime}\right\}$, the degree of $j$ is $\operatorname{deg}_{G}(j)=n-\sum_{k=1}^{n} a_{k j}$. If $i j$ is an edge of the bipartite representation of $J-A$, then $a_{i j}=0$ and so

$$
\operatorname{deg}_{G}(i)=n-\sum_{k=1}^{n} a_{i k} \geq n-\sum_{k=1}^{n} a_{k j}=\operatorname{deg}_{G}(j)
$$

Having shown all the conditions of Lemma 3.3.4 hold, we conclude that $\operatorname{deg}_{G}(i)=\operatorname{deg}_{G}(j)$ for all $i, j \in[n]$ such that $a_{i j}=0$. Thus row $i$ and column $j$ of $M(\overline{\mathcal{F}})$ have the same number of 1 s. That is, property (P3) holds. 
We are now ready prove two more of the key lemmas introduced in Section 3.2.1.

Proof of Lemma 3.2.3. Suppose for a contradiction that special point $\bar{x}$ of $P(\mathcal{F})$ is degenerate. Then $\bar{x}$ must have two distinct cores $M(\overline{\mathcal{F}})$ and $M\left(\overline{\mathcal{F}}^{\prime}\right)$ that differ in exactly one row. Lemma 3.3.5 implies that these cores have property $(\mathrm{P})$, contradicting Lemma 3.3.3.

Lemma 3.2.5. Suppose for a contradiction there exists special points $x^{\prime}$ and $x^{\prime \prime}$ that are adjacent. By Lemma 3.2.3, $x^{\prime}$ has a unique core $\mathcal{F}^{\prime}$ and $x^{\prime \prime}$ has a unique core $\mathcal{F}^{\prime \prime}$. Since $x^{\prime}$ and $x^{\prime \prime}$ are adjacent, $M\left(\mathcal{F}^{\prime}\right)$ and $M\left(\mathcal{F}^{\prime \prime}\right)$ differ in exactly one row. Lemma 3.3 .5 implies that these cores have property $(\mathrm{P})$, contradicting Lemma 3.3.3.

Having proved all the other lemmas of Section 3.2.1, it remains to prove Lemma 3.2.6.

\subsubsection{Proof of Lemma 3.2.6}

Two matrices are isomorphic to one another if one can be obtained from the other by permuting rows and columns. Lemma 4.12 in the proof of Lehman's Theorem we are following $[6]$ is equivalent to the following lemma.

Lemma 3.3.6. Let $Y$ and $Z$ be $n \times n$ matrices. Suppose $Y$ has property $(P)$, and suppose that

$$
Y Z^{T}=J+D
$$

where $D$ is a diagonal matrix with positive diagonal entries. Then either,

1. $Y$ and $Z$ are both isomorphic to $M\left(\mathcal{J}_{s}\right)$ for $s \geq 2$, or

2. $D=d I$, where $d$ is a positive integer.

A 0,1 -matrix $M$ is $r$-regular, if every row and every columns has exactly $r$ s. The following result is due to Bridges and Ryser [4].

Theorem 3.3.7. Let $Y$ and $Z$ be $n \times n, 0,1$-matrices such that $Y Z^{T}=J+d I$ for $d \geq 1$. Then $Y$ is $r$-regular, $Z$ is $\ell$-regular where $r, \ell \geq 2, n=r \ell-d$, and $Y Z^{T}=Z^{T} Y$.

Here, we differ from proof the of Lehman's Theorem we are following [6], only in that, we need a different argument to prove there are exactly $n$ minimum cardinality sets in $b(\mathcal{F})$. 
Proof Lemma 3.2.6. Lemma 3.2.3 implies that $\overline{\mathcal{F}}=\left\{S_{1}, \ldots, S_{n}\right\}$ is the unique core of $\bar{x}$. Denote by $b^{1}, \ldots, b^{n}$ the (integer) neighbours of $\bar{x}$ and let $Y$ be the $n \times n 0,1$-matrix for which row $i$ is the characteristic vector of $S_{i}$. For any $i \in[n], b^{i}$ and $\bar{x}$ are in a face of $P(\mathcal{F})$ of dimension 1 and so we may assume that the unique constraint of $Y x \geq \mathbb{1}$ that is not tight for $b^{i}$ is constraint $i$. Hence, if we denote by $Z$ the matrix with rows $b^{1}, \ldots, b^{n}$,

$$
Y Z^{T}=J+D
$$

where $D$ is a diagonal matrix with positive diagonal entries.

Lemma 3.3.5 implies that $Y$ has property $(\mathrm{P})$. Together with Theorem 3.3.6, it implies that either $Y$ is isomorphic to $M(\mathcal{J})$ or $D=d I$ for some integer $d \geq 1$. We may assume that the former case does not occur for otherwise we have the required outcome (1) of Lemma 3.2.6. By Theorem 3.3.7, $Y$ is $r$-regular, $Z$ is $\ell$-regular for $r, \ell \geq 2$. It follows that $\bar{x}=\frac{1}{r} \mathbb{1}$ and since $\bar{x}$ has a unique core, the clutter $\overline{\mathcal{F}}$ is exactly the minimum cardinality sets of $\mathcal{F}$.

Since the neighbours of $\bar{x}$ are $b^{1}, \ldots, b^{n}$ and since $b^{1}, \ldots, b^{n}$ have all exactly $\ell 1 \mathrm{~s}$, the constraint $\sum_{j=1}^{n} x_{j} \geq \ell$ is valid for all 0,1 points of $P(\mathcal{F})$. It follows, in particular, that every element in the blocker $\mathcal{K}$ of $\mathcal{F}$ has cardinality at least $\ell$. Let $\overline{\mathcal{K}}$ denote the minimum cardinality sets of $\mathcal{K}$. Then $\overline{\mathcal{K}}$ contains all sets corresponding to $b^{1}, \ldots, b^{n}$.

Suppose for a contradiction there exists a set $T \in \overline{\mathcal{K}}$ that does not correspond to any of $b^{1}, \ldots, b^{k}$ and let $b$ denote the characteristic vector of $T$. Since $T \in b(\mathcal{F}), b$ is an extreme point of $P(\mathcal{F})$ (see Remark 1.16 in [6]). Since $b$ is a linear combination of $b^{1}, \ldots, b^{n}$ (because $\left\{b^{1}, \ldots b^{n}\right\}$ has rank $\left.n\right)$, there exists vector $\alpha$ such that

$$
\sum_{j=1}^{n} \alpha_{j} b^{j}=b
$$

By right multiplying the previous equation on both sides by $\frac{1}{\ell} \mathbb{1}$ we obtain

$$
\sum_{j=1}^{n} \alpha_{j}=1
$$

Combining (3.4) and (3.5), we get

$$
\sum_{j=1}^{n} \alpha_{j}\left(b^{j}-\bar{x}\right)=b-\bar{x} .
$$


Since $b^{1}, \ldots, b^{n}$ are neighbours of $\bar{x}$ and since $b \in P(\mathcal{F}), b-\bar{x}$ is in the cone formed by the vectors $b^{1}-\bar{x}, \ldots, b^{n}-\bar{x}$, i.e. for some $\lambda \geq 0$

$$
\sum_{j=1}^{n} \lambda_{j}\left(b^{j}-\bar{x}\right)=b-\bar{x} .
$$

Subtracting (3.7) from (3.6) yields

$$
\sum_{j=1}^{n}\left(\alpha_{j}-\lambda_{j}\right)\left(b^{j}-\bar{x}\right)=0 .
$$

Since $P(\mathcal{F})$ is full dimensional, $b^{1}-\bar{x}, \ldots, b^{n}-\bar{x}$ are linearly independent. It follows from (3.8) that $\alpha=\lambda$ and in particular, $\alpha \geq 0$. Combining this with (3.4) and (3.5) shows $b$ is a convex combination of $b^{1}, \ldots, b^{n}$, contradicting the fact $b$ is an extreme point of $P(\mathcal{F})$.

\subsection{Exploring Lehman's Theorem}

Lehman's Theorem admits many combinatorial interpretations. In this section, an interpretation of the matrix equation $M(\overline{\mathcal{F}}) M(\overline{\mathcal{K}})^{T}=J+d I$ for two mni clutters is provided. Some of the many useful corollaries of Lehman's Theorem are stated and proven. All of the corollaries appearing here have been noted previously (see [18], [19], [28], for example).

\subsubsection{Interpreting Lehman's Theorem: $\mathcal{C}_{2,3}$ and $\mathcal{O}_{K_{5}}$}

The clutter $\mathcal{C}_{2,3}$ is minimally nonideal. Clutter $\mathcal{C}_{2,3}$ is the (non-binary) clutter on ground set $\{1,2,3\}$ with elements $\{\{1,2\},\{1,3\},\{2,3\}\}$. The blocker of $\mathcal{C}_{2,3}$ is itself; $b\left(\mathcal{C}_{2,3}\right)=\mathcal{C}_{2,3}$. The minimum cardinality elements of $\mathcal{C}_{2,3}$ are just $\mathcal{C}_{2,3}$, likewise for the blocker. Thus $M\left(\overline{\mathcal{C}_{2,3}}\right)\left[M\left(\overline{\left.b\left(\mathcal{C}_{2,3}\right)\right)}\right]^{T}=J+I\right.$. One way to interpret this matrix equation is to note that $F \in \mathcal{C}_{2,3}$ has intersection 2 with exactly one element in $b\left(\mathcal{C}_{2,3}\right)$ (the element corresponding to itself) and intersection 1 with the other two elements of $b\left(\mathcal{C}_{2,3}\right)$.

The clutter $\mathcal{O}_{K_{5}}$ is minimally nonideal. The minimum cardinality elements of $\mathcal{O}_{K_{5}}$ are the triangles of $K_{5}$. The minimum cardinality elements of $b\left(\mathcal{O}_{K_{5}}\right)$ are the union of a triangle of $K_{5}$ together with the non-adjacent edge. Thus $M\left(\overline{\mathcal{O}_{K_{5}}}\right)\left[M\left(\overline{b\left(\mathcal{O}_{K, 5}\right)}\right)\right]^{T}=J+2 I$. One way to interpret this matrix equation is to note that each triangle $T$ of $K_{5}$ has intersection 3 with the element of the blocker consisting of $T$ and the opposite edge and intersection 1 with every other minimum cardinality blocker element. 


\subsubsection{Useful Corollaries}

Given clutter $\mathcal{F}$ we denote by $\overline{\mathcal{F}}$ the clutter formed by the sets of minimum cardinality in $\mathcal{F}$. Let $(\mathcal{F}, \mathcal{K})$ be a blocking pair that is Lehman. We say that $F \in \overline{\mathcal{F}}$ and $K \in \overline{\mathcal{K}}$ are mates if $|F \cap K| \geq 2$. Equation (3.1) implies every $F \in \overline{\mathcal{F}}$ has a unique mate $K$ and $|F \cap K|=r \ell-n+1:=q$. Moreover, if $F \in \overline{\mathcal{F}}$ and $K^{\prime} \in \overline{\mathcal{K}}$ are not mates then $\left|F \cap K^{\prime}\right|=1$. Since $\overline{\mathcal{F}}$ is binary, elements of $\overline{\mathcal{F}}$ and $\overline{\mathcal{K}}$ intersect with odd parity and thus $|F \cap K| \geq 3$.

Interpreting equation (3.1) combinatorially leads to many corollaries of Lehman's Theorem. First note that $M(\overline{\mathcal{F}})$ and $[M(\overline{\mathcal{K}})]^{T}$ commute (Theorem 3.3.7) and $(J+d I)^{T}=J+d I$; thus $M[\overline{\mathcal{F}}]^{T} M[\overline{\mathcal{K}}]=J+d I$.

Corollary 3.4.1. Let $e \in E(\mathcal{F})$ be a column index of $M[\overline{\mathcal{K}}]$. Let $F_{1}, \ldots F_{\ell}$ be the characteristic sets of the rows of $M[\overline{\mathcal{F}}]$ whose indices are given by the characteristic set of column e of $M[\overline{\mathcal{K}}]$. Then $F_{1} \ldots F_{\ell}$ pairwise intersect in at most $\{e\} ;$ moreover, exactly $q$ of $F_{1} \ldots F_{\ell}$ contain $\{e\}$. Lastly, $E=F_{1} \cup \ldots \cup F_{\ell}$.

Proof. Since $M[\overline{\mathcal{F}}]^{T} M[\overline{\mathcal{K}}]$, for each column index $j$ of $M[\overline{\mathcal{K}}]$, we have $M[\overline{\mathcal{F}}]^{T} \operatorname{col}(M[\overline{\mathcal{K}}], j)=$ $\mathbb{1}+d e_{j}$ where $e_{j}$ is the $j$ th standard basis vector and $\operatorname{col}(M[\overline{\mathcal{K}}], j)$ denotes the $j$ th column of $M[\overline{\mathcal{K}}]$. The corollary follows.

Corollary 3.4.2. Let $e \in E(\mathcal{F})$ be a column index of $M[\overline{\mathcal{F}}]$. Let $K_{1}, \ldots K_{r}$ be the characteristic sets of the rows of $M[\overline{\mathcal{K}}]$ whose indices are given by the characteristic set of column e of $M[\overline{\mathcal{F}}]$. Then $K_{1} \ldots K_{r}$ pairwise intersect in at most $\{e\} ;$ moreover, exactly $q$ of $K_{1} \ldots K_{r}$ contain $\{e\}$. Lastly, $E=K_{1} \cup \ldots \cup K_{r}$.

Proof. Similar to proof of Corollary 3.4.1.

Corollary 3.4.3. For each $e \in E$ there are precisely $q$ pairs $\left\{\left(F_{1}, K_{1}\right), \ldots\left(F_{q}, K_{q}\right)\right\}$ such that $e \in F_{i} \cap K_{i}$. For each distinct $e, f \in E$, there is exactly one pair $(F, K)$ with $e \in F$, $f \in K$.

Proof. This proof appears in [28]. Let $e \in E$. Since $(M(\overline{\mathcal{F}}))[M(\overline{\mathcal{K}})]^{T}$, column $e$ of $M(\overline{\mathcal{K}})$ must have exactly $q$ 1s in rows corresponding to the characteristic set of column $e$ of $M(\overline{\mathcal{F}})$. Moreover, column $f$ must have exactly one 1 in the rows corresponding to the characteristc set of column $e$ of $M(\overline{\mathcal{F}})$.

Corollary 3.4.4. For fixed $e \in E$, if $\left\{\left(F_{1}, K_{1}\right), \ldots\left(F_{q}, K_{q}\right)\right\}$ are the mates such that $e \in$ $F_{i} \cap K_{i}$ then $\left\{F_{1}, \ldots F_{q}, K_{1} \ldots K_{q}\right\}$ pairwise intersect in at most $\{e\}$ except for $\left|F_{i} \cap K_{i}\right|=q$. 
Proof. Now $F_{i} \cap B_{j}=\{e\}$ since $\left|C_{i} \cap B_{j}\right|=1$ for all distinct $i, j \in\{1 \ldots q\} \subseteq\{1 \ldots n\}$. Similarly, the facts $\left|F_{i} \cap B_{i}\right|=q$ and $e \in F_{i} \cap B_{i}$ let us conclude that $\left|\left(F_{i} \cap K_{i}\right)\right|=q$. It remains to show that $C_{i} \cap C_{j}=\{e\}$ for all distinct $i, j \in\{1, \ldots q\}$ and $B_{i} \cap B_{j}=\{e\}$ for all distinct $i, j \in\{1, \ldots q\}$. Suppose there exists $f \in E-e$ such that $f \in C_{i} \cap C_{j}$. Then $e \in B_{i}$ and $f \in C_{i}$, and $e \in B_{j}$ and $f \in C_{j}$. This contradicts the second conclusion of Corollary 3.4.3. Therefore such $f$ cannot exist. Thus $C_{i} \cap C_{j}=\{e\}$ for all distinct $i, j \in\{1 \ldots q\}$. The analysis is analogous for showing $B_{i} \cap B_{j}=\{e\}$ for distinct $i, j \in\{1 \ldots q\}$.

Corollary 3.4.5. There exists no set $K$ such that $|K \cap F|=1$ for all $F \in \overline{\mathcal{F}}$.

Proof. By way of contradiction, suppose there exists $K$ such that $|K \cap F|=1$ for all $F \in \overline{\mathcal{F}}$. By Corollary 3.4.1, for $e \in K$, there exists a collection of sets $\left\{F_{1} \ldots F_{\ell}\right\}$ such that $E=F_{1} \cup \ldots \cup F_{\ell}$ and $e \in F_{1} \cap F_{2}$. This implies $|K| \leq \ell-1$. Again by Corollary 3.4.1, for $e^{\prime} \notin K$, there exists a collection of sets $\left\{K_{1}^{\prime} \ldots K_{\ell}^{\prime}\right\}$ that pairwise intersect in at most $\{e\}$. This implies $|K| \geq \ell$, a contradiction. Conclude that no such $K$ can exist.

The following results appear in [18] and apply only to binary clutters. The statements were given for minimally nonideal clutters, but the proof works for the more general class of Lehman clutters.

Corollary 3.4.6. Let $(\mathcal{F}, \mathcal{K})$ be a blocking pair that is binary and Lehman, and let $e \in$ $E(\mathcal{F})$. Then there exists $F_{1}, F_{2}, F_{3} \in \overline{\mathcal{F}}$ that pairwise intersect exactly in e. Moreover, the mates $K_{1}, K_{2}, K_{3}$ of $F_{1}, F_{2}, F_{3}$ pairwise intersect exactly in e.

Proof. This proof appears in [19]. Since $q \geq 3$ because $\mathcal{F}$ is binary, result follows from Corollary 3.4.4.

Corollary 3.4.7. Let $(\mathcal{F}, \mathcal{K})$ be a blocking pair that is binary and Lehman.

1. For all $F_{1}, F_{2} \in \overline{\mathcal{F}}$ and $F \in \mathcal{F}$ where $F \subseteq F_{1} \cup F_{2}$ either $F=F_{1}$ or $F=F_{2}$.

2. For all $K_{1}, K_{2} \in \overline{\mathcal{K}}$ and $K \in \mathcal{K}$ where $K \subseteq K_{1} \cup K_{2}$ either $K=K_{1}$ or $K=K_{2}$.

Proof. Since $\mathcal{F}$ is binary, there exists $F^{\prime} \subseteq F_{1} \triangle F_{2} \triangle F$ such that $F^{\prime} \in \mathcal{F}$. If $e \in F \cap F^{\prime}$ and $F_{1} \triangle F_{2} \triangle F$, then $e \notin F_{1} \triangle F_{2}$. Thus $F \cup F^{\prime} \subseteq F_{1} \cup F_{2}$ and $F \cap F^{\prime} \subseteq F_{1} \cap F_{2}$. It follows that $|F|+\left|F^{\prime}\right| \leq\left|F_{1}\right|+\left|F_{2}\right|$ and thus $F, F^{\prime} \in \mathcal{F}$. Let $B$ be the mate of $F$. Since $\mathcal{F}$ is binary, $|B \cap F|$ must be odd and hence it is at least 3. Then either $\left|F_{1} \cap B\right| \geq 2$ or $\left|F_{2} \cap B\right| \geq 2$ and since $B$ has a unique mate in $\overline{\mathcal{F}}$, it must be that $F=F_{1}$ or $F=F_{2}$. Similarly, one can show that if $F=F_{1}$, then $F^{\prime}=F_{2}$ and if $F=F_{2}$, then $F^{\prime}=F_{1}$. The proof is the same for $K_{1}, K_{2}, K \in \overline{\mathcal{K}}$. 
In the remainder of this thesis, an above corollary will be referenced by "Lehman Corollary" followed by the corollary number. 


\section{Chapter 4}

\section{Applications to Odd-st-Walks and st-T-Cuts}

\subsection{Chapter Overview}

This chapter focuses on two cases: $(1) \mathcal{F}$ is a clutter of odd-st-walks, and, (2) $\mathcal{F}$ is a clutter of st-T-cuts.

Given signed graph $(G, \Sigma), s, t \in V(G)$, and weight function $w \in \mathbb{Z}_{+}^{E(G)}$, let $\mathcal{F}$ be the clutter of odd-st-walks of $(G, \Sigma)$. Whenever $\mathcal{F}$ has no $\mathcal{O}_{K_{5}}$ or $\mathcal{F}_{7}$ minor, there exists a fractional $w$-packing and integer $w$-cover of $\mathcal{F}$ with the same value (Theorem 2.4.1). Using the constructive version of Lehman's Theorem, one can either find a fractional w-packing and integer $w$-cover of the same value or show that $\mathcal{O}_{K_{5}}$ or $\mathcal{L}_{7}$ is a minor of $\mathcal{F}$. Moreover, this can be done in polynomial time, proving below Theorem 4.1 .1 (which is equivalent to Theorem 1.3.2).

Theorem 4.1.1. Let $(G, \Sigma)$ be a signed graph and $w \in \mathbb{Z}_{+}^{E(G)}$. Let $s, t \in V(G)$ and $\mathcal{F}$ be the clutter of odd-st-walks of $(G, \Sigma)$. Then in time polynomial in $|V(G)|+\langle w\rangle$, one can either find

1. $I, J \subseteq E(\mathcal{F})$ such that $\mathcal{F} / I \backslash J$ is isomorphic to $\mathcal{O}_{K_{5}}$ or $\mathcal{L}_{7}$, or

2. an integer solution to (2.5) and a solution to (2.6) with the same value.

Given graft $(G, T), s, t \in V(G)$, and weight function $w \in \mathbb{Z}_{+}^{E(G)}$, let $\mathcal{F}$ be the clutter of st-T-cuts of $(G, T)$. Whenever $\mathcal{F}$ has no $b\left(\mathcal{O}_{K_{5}}\right)$ or $\mathcal{L}_{7}$ minor, there exists a fractional 
$w$-packing and integer $w$-cover of $\mathcal{F}$ with the same value (Theorem 2.4.4). Using the constructive version of Lehman's Theorem, one can either find a fractional $w$-packing and integer $w$-cover of the same value or show that $b\left(\mathcal{O}_{K_{5}}\right)$ of $\mathcal{L}_{7}$ is a minor of $\mathcal{F}$. Moreover, this can be done in polynomial time, proving below Theorem 4.1.2 (which is equivalent to Theorem 1.3.3).

Theorem 4.1.2. Let $(G, T)$ be a graft and $w \in \mathbb{Z}_{+}^{E(G)}$. Let $s, t \in V(G)$ and $\mathcal{F}$ be the clutter of st-T-cuts in $(G, T)$. Then in time polynomial in $|V(G)|+\langle w\rangle$, one can either find

1. $I, J \subseteq E(\mathcal{F})$ such that $\mathcal{F} / I \backslash J$ is isomorphic to $b\left(\mathcal{O}_{K_{5}}\right)$ or $\mathcal{L}_{7}$, or

2. an integer solution to (2.5) and a solution to (2.6) with the same value.

This chapter is dedicated to proving Theorems 4.1.1 and 4.1.2. Section 4.2 introduces signed graph and graft minor operations and shows them correspond to minor operations for clutters of odd-st-walks and clutters of $s t-T$-cuts respectively. Section 4.3 provides a proof outline; the remaining sections include the more technical details.

\subsection{Minors in Signed Graphs and Grafts}

Let $(G, \Sigma)$ be a signed graph with $s, t \in V(G)$. Although minor operations in signed graphs have already been defined, here we must take care to retain $s$ and $t$. Updating $\Sigma$ to $\Sigma \triangle \delta(S)$ for $s, t \in S \subseteq V$ preserves the set of odd $s t$-walks in $(G, \Sigma)$. We call this operation resigning and refer to any $\Sigma^{\prime}$ obtained in this way as a signature of $(G, \Sigma)$. Since $(G, \Sigma)$ and $\left(G, \Sigma^{\prime}\right)$ have the same set of odd $s$-walks, we will view these two signed graphs as equivalent. Accordingly, we do not account explicitly for the resigning operation. Given $(G, \Sigma)$ and $e \in E$, we define $(G, \Sigma) \backslash e$ as $(G \backslash e, \Sigma-e)$ and call this operation deletion. Given $(G, \Sigma)$ and $e \in E$ that is not an odd loop, suppose we resign to obtain $\Sigma^{\prime}$ such that $e \notin \Sigma^{\prime}$. We define $(G, \Sigma) / e$ as $\left(G / e, \Sigma^{\prime}\right)$ and call this operation contraction. If contracting edge $e=s x$, call the resulting vertex $s$. If contracting edge $e=t x$, call the resulting vertex $t$. If contracting edge $e=s t$, call the resulting vertex $s=t$. Any $\left(G^{\prime}, \Sigma^{\prime}\right)$ obtained by applying a sequence of deletions and contractions is called a minor of $(G, \Sigma)$.

We make some notes about this definition. Minor operations can be applied in any order and so we unambiguously denote by $(G, \Sigma) / I \backslash J$ the signed graph obtained by contracting all edges in $I \subseteq E$ and deleting all edges in $J \subseteq E$ (and resigning in whatever way avoids contracting signature edges). Necessarily $I$ does not contain an odd circuit. Note that if $s=t$, the minor operations defined here correspond exactly to the signed graph 
minor operations defined in Section 1.1.3. The following proposition shows performing contractions and deletions in a signed graph corresponds to performing the same operations in the underlying clutter of odd-st-walks.

Proposition 4.2.1. If $\mathcal{F}$ is the clutter of odd st-walks of $(G, \Sigma)$ then $\mathcal{F} / I \backslash J$ is the clutter of odd st-walks of $(G, \Sigma) / I \backslash J$.

Proof. It suffices to show that $\mathcal{F} \backslash e$ is the clutter of odd-st-walks of $(G, \Sigma) \backslash e$ and $\mathcal{F} / e$ is the clutter of odd-st-walks of $(G, \Sigma) / e$.

The clutter $\mathcal{F} \backslash e$ is defined as $\{F \in \mathcal{F}: e \notin F\}$. In other words $\mathcal{F} \backslash e$ contains exactly the odd-st-walks of $(G, \Sigma)$ that do not use edge $e$. Deleting edge $e$ from $(G, \Sigma)$ results in removing all odd-st-walks that use $e$ and preserving all other odd-st-walks. Therefore, $\mathcal{F} \backslash e$ is indeed the clutter of odd-st-walks of $(G, \Sigma) \backslash e$.

Without loss of generality, assume $e \notin \Sigma$ since otherwise we can resign and preserve the set of odd-st-walks. The clutter $\mathcal{F} / e$ is defined as the minimal sets of $\{F-e: F \in \mathcal{F}\}$. In other words, sets of $\mathcal{F} / e$ come in two sorts: odd $s t$-walks of $(G, \Sigma)$ that do not use $e$ and odd-st-walks of $(G, \Sigma)$ using $e$ with the edge $e$ removed. Since $e \notin \Sigma$, the parity of $D \subseteq E$ is the same in both $(G, \Sigma)$ and $(G, \Sigma) / e$. Thus, contracting $e$ in $(G, \Sigma)$ preserves the odd-st-walks that do not use $e$. Since $e \notin \Sigma$, if $F$ is an odd-st-walk using $e$, then $F-e$ is an odd-st-walk of $(G, \Sigma) / e$. Therefore, $\mathcal{F} / e$ is indeed the clutter of odd-st-walks of $(G, \Sigma) / e$.

Minor operations can also be defined for grafts. Let $(G, T)$ be a graft with $s, t \in V(G)$. Suppose $e \in E(G) \backslash\{s t\}$ is not an odd bridge (an edge whose removal breaks $(G, T$ ) into two components each of which contains an odd number of $T$ vertices). We define $(G, T) \backslash e$ as $(G \backslash e, T)$ and call this operation deletion. For $e=x y \in E(G)$, let $G^{\prime}=G / e$. If $z$ is the vertex obtained by contracting $e$, then let $T^{\prime}=T-\{x, y\} \cup\{z\}$ if exactly one of $x, y$ is in $T$ and otherwise let $T^{\prime}=T-\{x, y\}$. We define $(G, T) / e$ as $\left(G^{\prime}, T^{\prime}\right)$ and call this operation contraction. If contracting edge $e=s x$, call the resulting vertex $s$. If contracting edge $e=t x$, call the resulting vertex $t$. Any $\left(G^{\prime}, T^{\prime}\right)$ obtained by applying a sequence of deletions and contractions is called a minor of $(G, T)$.

Again, minor operations can be applied in any order and so we can unambiguously denote by $(G, T) / I \backslash J$ the graft obtained by contracting all edges in $I \subseteq E$ and deleting all edges in $J \subseteq E$. Necessarily $G \backslash I$ does not contain a $T$-odd component. The following proposition shows contraction in the graft corresponds to deletion in the underlying clutter of $s t-T$-cuts and deletion in the grafts corresponds to contraction in the underlying clutter of $s t$ - $T$-cuts. 
Proposition 4.2.2. If $\mathcal{F}$ is the clutter of st-T-cuts of $(G, T)$ then $\mathcal{F} / I \backslash J$ is the clutter of st-T-cuts of $(G, T) \backslash I / J$.

Proof. It suffices to show that $\mathcal{F} \backslash e$ is the clutter of st-T-cuts of $(G, T) / e$ and $\mathcal{F} / e$ is the clutter of st-T-cuts of $(G, \Sigma) \backslash e$.

The clutter $\mathcal{F} \backslash e$ is defined as $\{F \in \mathcal{F}: e \notin F\}$. In other words, $\mathcal{F} \backslash e$ contains exactly the st-T-cuts of $(G, T)$ that do not use $e$. Suppose $\delta(S)$ for $S \subseteq V$ is an st-T-cut of $(G, T)$ and let $\left(G^{\prime}, T^{\prime}\right)=(G, T) / e$. If $e \in \delta(S)$, there is no corresponding st-T'-cut of $\left(G^{\prime}, T^{\prime}\right)$ since $\delta(S) \backslash e$ is not a cut of $\left(G^{\prime}, T^{\prime}\right)$. If $e=x y \notin \delta(S)$, then $\delta(S)$ is a st- $T^{\prime}$-cut of $\left(G^{\prime}, T^{\prime}\right)$. Moreover, either $x, y \in S$ or $x, y \notin S$. In both cases, $\left|S \cap T^{\prime}\right|$ is odd since contracting $\{x, y\}$ to $\{z\}$ is defined so that the parity of $T \cap\{x, y\}$ and the parity of $T^{\prime} \cap\{z\}$ are the same. Thus $\delta(S)$ is an st- $T^{\prime}$-cut of $\left(G^{\prime}, T^{\prime}\right)$.

The clutter $\mathcal{F} / e$ is defined as the minimal elements of $\{F-e: F \in \mathcal{F}\}$. In other words, sets of $\mathcal{F} / e$ come in two sorts: st-T-cuts of $(G, T)$ that do not use $e$ and st-T-cuts of $(G, T)$ using $e$ with the edge $e$ removed. If $\delta(S)$ is an st-T-cut of $(G, T)$ that does not use $e$, it is clear the $\delta(S)$ is an st-T-cut of $(G, T) \backslash e$ that does not use $e$. Similarly, if $\delta(S)$ is an st-T-cut of $(G, T)$ using $e, \delta(S) \backslash e$ is an st-T-cut of $(G, T) \backslash e$.

It follows that we can consider signed graph and graft minor operations instead of clutter operations whenever it is convenient.

\subsection{Proof Outline}

The proofs for Theorem 4.1.1 (regarding clutters of odd-st-walks) and Theorem 4.1.2 (regarding clutters of $s t$ - $T$-cuts) follow the same outline. In the interest of clarity and at the cost of repetition, full outlines of both proofs follow.

\subsubsection{Proof Outline: Theorem 4.1.1}

Recall that the separation problem for $P$ and a point $\bar{x} \in \mathbb{Q}^{n}$ is to either determine that $\bar{x} \in P$, or to find a separating constraint $a^{T} x \leq \beta$ (i.e., $a^{T} \bar{x}>\beta$ and $a^{T} x \leq \beta$ for all $x \in P$ ). In order for the constructive version of Lehman's Theorem (Theorem 3.1.3) to give a polynomial time algorithm, the separation problem for $Q(\mathcal{F})$ must be solvable in polynomial time. For odd-st-walk covering polytopes, polynomial time separation follows almost immediately from being able to find minimum length circuits and paths in graphs 
subject to a parity condition (see [16]). Section 4.4 describes the separation algorithm and shows correctness; the following proposition appears.

Proposition 4.3.1. Let $(G, \Sigma)$ be a signed graph and let $s, t \in V(G)$. Let $\mathcal{F}$ be the clutter of odd st-walks of $(G, \Sigma)$. Given $\bar{x} \in \mathbb{Q}^{E(G)}$, we can solve the separation problem for $\bar{x} \in \mathbb{Q}^{n}$ and $Q(\mathcal{F})$ in time polynomial in $|V(G)|+\langle\bar{x}\rangle$.

The following proposition is obtained by combining (6.5.9) and (6.5.15) in [16] and allows us to find not only optimal solutions to the packing and covering LPs, but also defining constraints.

Proposition 4.3.2. Let $\mathcal{F}$ be a clutter and suppose that $Q(\mathcal{F}) \subseteq \mathbb{R}^{n}$ is given by a separation oracle. If $w \in \mathbb{Z}_{+}^{E(\mathcal{F})}$, then in oracle polynomial time in $n+\langle w\rangle$ we can find an optimal solution $\bar{y}$ to (2.6) and an extreme point $\bar{x}$ of $Q(\mathcal{F})$ that is optimal for (2.5) together with a set of $n$ constraints of $Q(\mathcal{F})$ defining $\bar{x}$.

Let $\mathcal{F}$ be the clutter of odd $s t$-walks of $(G, \Sigma)$. Since the separation problem for $\mathcal{F}$ is polynomially solvable by Proposition 4.3.1, it follows immediately from Proposition 4.3.2 that we can find in time polynomial in $|V(G)|+\langle w\rangle$

1. an optimal solution $\bar{y}$ to (2.6) (the odd-st-walk packing LP),

2. an extreme point $\bar{x}$ of $Q(\mathcal{F}) \equiv(2.5)$ (the odd-st-walk covering LP), and,

3. a set of $n$ constraints of $Q(\mathcal{F})$ defining $\bar{x}$.

If $\bar{x}$ is integral, we have achieved the second outcome of Theorem $4.1 .1 ; \bar{x}$ and $\bar{y}$ are an integer $w$-cover and fractional $w$-packing of odd-st-walks of $(G, \Sigma)$ with the same value. Otherwise, we aim to find $I, J \subseteq E(\mathcal{F})$ such that $\mathcal{F} / I \backslash J$ is isomorphic to $\mathcal{O}_{K_{5}}$ or $\mathcal{L}_{7}$. Proposition 4.3.1 gives a polynomial time separation oracle for $Q(\mathcal{F}), \bar{x}$ is a fractional extreme point of $Q(\mathcal{F})$, and we know $n$ facets of $Q(\mathcal{F})$ that define $\bar{x}$. Hence the constructive version of Lehman's Theorem (Theorem 3.1.3) applies; in polynomial time we can find $I, J \subseteq E(\mathcal{F})$ such that $\mathcal{F}^{\prime}=\mathcal{F} / I \backslash J$ is a Lehman clutter. The first outcome $\left(\mathcal{F}^{\prime}=\mathcal{J}_{s}\right.$ for $s \geq 2$ ) cannot occur since the clutter of odd-st-walks is binary but $\mathcal{J}_{s}$ is not. Theorem 3.1.3 also lets us find the minimum cardinality sets of $\mathcal{F}^{\prime}$. By Proposition 4.2.1, $\mathcal{F}^{\prime}$ is the clutter of odd-st-walks of $\left(G^{\prime}, \Sigma^{\prime}\right)=(G, \Sigma) / I \backslash J$.

Given Lehman clutter $\mathcal{F}^{\prime}$, Lemma 4.3 .3 below proves we can efficiently show $\mathcal{O}_{K_{5}}$ or $\mathcal{L}_{7}$ is a minor of $\mathcal{F}^{\prime}$. The proof is a constructive variant of known structures (see [28] and [19]). A proof of Lemma 4.3.3 appears in Section 4.5.

Lemma 4.3.3. Let $(G, \Sigma)$ be a signed graph and let $s, t \in V(G)$. Let $\mathcal{F}$ be the clutter of odd st-walks of $(G, \Sigma)$. Suppose that $\mathcal{F}$ is a Lehman clutter and that we are given the minimum 
cardinality sets of $\mathcal{F}$. Then in time polynomial in $|V(G)|$ we can find $I, J \subseteq E(\mathcal{F})$ such that $\mathcal{F} / I \backslash J$ is isomorphic to $\mathcal{O}_{K_{5}}$ or $\mathcal{L}_{7}$.

\subsubsection{Proof Outline: Theorem 4.1.2}

Polynomial time separation for st-T-cut covering polytopes was known previously (see [16]). Section 4.4 describes the separation algorithm and shows correctness; the following proposition appears.

Proposition 4.3.4. Let $(G, T)$ be a graft and let $s, t \in V(G)$. Let $\mathcal{F}$ be the clutter of st-T-cuts of $(G, \Sigma)$. Given any $\bar{x} \in \mathbb{Q}^{E(G)}$, we can solve the separation problem for $\bar{x} \in \mathbb{Q}^{n}$ and $Q(\mathcal{F})$ in time polynomial in $|V(G)|+\langle\bar{x}\rangle$.

Let $\mathcal{F}$ be the clutter of $s t$ - $T$-cuts of $(G, T)$. Since the separation problem for $\mathcal{F}$ is polynomially solvable by Proposition 4.3.4, it follows immediately from Proposition 4.3.2 we can find in time polynomial in $|V(G)|+\langle w\rangle$

1. an optimal solution $\bar{y}$ to (2.6) (the st-T-cut packing LP),

2. an extreme point $\bar{y}$ of $Q(\mathcal{F}) \equiv(2.5)$ (the st-T-cut covering LP), and,

3. a set of $n$ constraints of $Q(\mathcal{F})$ defining $\bar{x}$.

If $\bar{x}$ is integral, we have achieved the second outcome of Theorem $4.1 .2 ; \bar{x}$ and $\bar{y}$ are an integer $w$-cover and fractional $w$-packing of $s t$ - $T$-cuts of $(G, T)$ with the same value. Otherwise, we aim to find $I, J \subseteq E(\mathcal{F})$ such that $\mathcal{F} / I \backslash J$ is isomorphic to $b\left(\mathcal{O}_{K_{5}}\right)$ or $\mathcal{L}_{7}$. Proposition 4.3.4 gives a polynomial time separation oracle for $Q(\mathcal{F}), \bar{x}$ is a fractional extreme point of $Q(\mathcal{F})$, and we know $n$ facets of $Q(\mathcal{F})$ that define $\bar{x}$. Hence the constructive version of Lehman's Theorem (Theorem 3.1.3) applies; in polynomial time we can find $I, J \subseteq E(\mathcal{F})$ such that $\mathcal{F}^{\prime}=\mathcal{F} / I \backslash J$ is a Lehman clutter. The first outcome $\left(\mathcal{F}^{\prime}=\mathcal{J}_{s}\right.$ for $s \geq 2$ ) cannot occur since the clutter of $s t-T$-cuts is binary but $\mathcal{J}_{s}$ is not. Theorem 3.1.3 also lets us find the minimum cardinality sets of $\mathcal{F}^{\prime}$. By Proposition 4.2.2, $\mathcal{F}^{\prime}$ is the clutter of st-T-cuts of $\left(G^{\prime}, T^{\prime}\right)=(G, T) / J \backslash I$.

Given Lehman clutter $\mathcal{F}^{\prime}$, Lemma 4.3 .5 below proves we can efficiently show $b\left(\mathcal{O}_{K_{5}}\right)$ or $\mathcal{L}_{7}$ is a minor of $\mathcal{F}^{\prime}$. The proof is a constructive variant of known structures (see [19]). A proof of Lemma 4.3.5 appears in Section 4.6.

Lemma 4.3.5. Let $(G, T)$ be a graft and let $s, t \in V(G)$. Let $\mathcal{F}$ be the clutter of st-T-cuts of $G$. Suppose that $\mathcal{F}$ is a Lehman clutter and that we are given the minimum cardinality sets of $\mathcal{F}$. Then in time polynomial in $|V(G)|$ we can find $I, J \subseteq E(\mathcal{F})$ such that $\mathcal{F} / I \backslash J$ is isomorphic to $b\left(\mathcal{O}_{K_{5}}\right)$ or $\mathcal{L}_{7}$. 


\subsection{Solving the Separation Problems}

\subsubsection{The Separation Problem for Odd-st-Walks}

Let $(G, \Sigma)$ be a signed graph, $s, t \in V(G)$, and $\mathcal{F}$ be the clutter of odd $s t$-walks of $(G, \Sigma)$. Given $\bar{x} \in \mathbb{Q}^{n}$, we require a polynomial time method to show $\bar{x} \in Q(\mathcal{F})$ or find a separating hyperplane.

It is straightforward to check that $0 \leq \bar{x}_{e} \leq 1$ for all $e \in E$ in polynomial time. We also need to verify $\sum\left(\bar{x}_{e}: e \in F\right) \geq 1$ for all $F \in \mathcal{F}$. Since $\mathcal{F}$ may have exponentially many members, we can not verify all of these constraints in the straightforward way. Suppose $F^{*} \in \mathcal{F}$ is a minimum weight odd-st-walk with respect to weight function $\bar{x}$. If $\sum\left(\bar{x}_{e}: e \in F^{*}\right)<1$, then letting $a$ be the characteristic vector of $F^{*}$ and $b$ be $1, a^{T} x \geq b$ is a separating hyperplane for $\bar{x}$ and $Q(\mathcal{F})$. If $\sum\left(\bar{x}_{e}: e \in F^{*}\right) \geq 1$ then for all $F \in \mathcal{F}$

$$
\sum\left(\bar{x}_{e}: e \in F\right) \geq \sum\left(\bar{x}_{e}: e \in F^{*}\right) \geq 1
$$

where the first inequality holds because $F^{*}$ is a minimum weight member of $\mathcal{F}$. Therefore, we can conclude $\bar{x} \in Q(\mathcal{F})$. It remains to show that we can find a minimum weight odd $s t$-walk in polynomial time.

This problem is closely related to finding a minimum weight circuit or path in a graph subject to a parity condition. In graph $G=(V, E)$, the parity of $E^{\prime} \subseteq E$ is simply $\left|E^{\prime}\right|$, while in a signed graph $(G, \Sigma)$, the parity of $E^{\prime} \subseteq E$ is $\left|E^{\prime} \cap \Sigma\right|$. However, finding an odd circuit in $(G, \Sigma)$ is reducible to finding an odd circuit in $G^{\prime}$ where $G^{\prime}$ is obtained from $G$ by replacing every edge $e \notin \Sigma$ by a path of length 2 . Therefore, a polynomial time algorithm for finding minimum weight circuits and paths in graphs subject to a parity condition implies a polynomial time algorithm for finding minimum weight circuits and paths in signed graphs subject to a parity condition. Algorithms for these problems in graphs are known [16]. For completeness, a precise statement and proof follow.

Theorem 4.4.1. Given a graph $G=(V, E), s, t \in V(G)$, and weight function $x \in \mathbb{Q}_{+}^{E}$, the following problems are solvable in polynomial time.

1. Find a minimum weight odd circuit through fixed $s \in V$.

2. Find a minimum weight odd circuit in $G$.

3. Find a minimum weight even circuit in $G$.

4. Find a minimum weight even st-path in $G$.

5. Find a minimum weight odd st-path in $G$. 
Proof. Let the auxiliary graph $G^{\prime}$ be obtained from $G$ by splitting each vertex $v \in V$ into vertices $v_{1}$ and $v_{2}$. If edge $e=x y \in E(G)$, then add edges $x_{1} y_{2}$ and $x_{2} y_{1}$ to $E\left(G^{\prime}\right)$, giving both weight $w_{e}$. Note that $G^{\prime}$ can be computed in polynomial time.

The shortest odd circuit through fixed $s$ in $G$ is the shortest $s_{1}, s_{2}$ path in $G^{\prime}$. Since this shortest path can be found in polynomial time (for example, by Dijkstra's algorithm), finding a minimum weight odd circuit through $s$ can be done in polynomial time.

Apply the algorithm for finding a minimum weight circuit $C_{s}$ through fixed $s \in V$ to each vertex to find a minimum weight odd circuit in $G$ by simply returning $C=\operatorname{argmin}\left\{C_{v}\right.$ : $v \in V\}$. Since this requires $n$ applications of a polynomial time algorithm, this too runs in polynomial time.

The minimum weight even circuit in $G$ must use some edge $e \in E(G)$. Note that if $\tilde{C}$ is an even circuit, then $\tilde{C} \backslash e$ is an odd path. Thus, the minimum weight even circuit problem can be solved by finding for each $e=x y \in E$ the shortest odd $x y$-path.

Auxiliary graph $G^{\prime}$ can be used to find the shortest odd and even paths in $G$. To find a minimum weight even path from $s$ to $t$, find a shortest path from $s_{1}$ to $t_{1}$. To find a minimum weight odd path from $s$ to $t$, find a shortest path from $s_{1}$ to $t_{2}$. Thus, a minimum weight even st-path, minimum weight odd st-path and so also a minimum weight even cycle can be found in polynomial time.

Finding a minimum weight odd-st-walk in polynomial time follows almost immediately from the preceding theorem.

Claim 4.4.2. Given $(G, \Sigma)$ such that $s, t \in V(G)$ and weight function $x \in \mathbb{Q}_{+}^{E}$, there exists a polynomial time algorithm to find a minimum weight odd-st-walk of $(G, \Sigma)$.

Proof. Recall that an odd st-walk is either an odd st-path or the edge disjoint union of an even st-path $P$ and an odd circuit $C$ such that $V(P)$ and $V(C)$ have at most one vertex in common. Suppose $P_{\text {odd }}$ is a minimum weight odd st-path in $(G, \Sigma), P_{\text {even }}$ is a minimum weight even st-path in $(G, \Sigma)$, and $C_{o d d}$ is a minimum weight odd circuit in $(G, \Sigma)$.

If $w\left(P_{\text {odd }}\right) \leq w\left(P_{\text {even }} \cup C_{\text {odd }}\right)$ then $P_{\text {odd }}$ is the minimum odd st-walk since any odd $s t$ walk that is the union of an even path and odd circuit must have weight at least $w\left(P_{\text {even }}\right)+$ $w\left(C_{\text {odd }}\right)$.

Otherwise $w\left(P_{\text {even }} \cup C_{\text {odd }}\right) \leq w\left(P_{\text {odd }}\right)$. If $P_{\text {even }} \cup C_{\text {odd }}$ is the edge disjoint union of an odd circuit and even st-path sharing at most one vertex, then $P_{\text {even }} \cup C$ is an odd $s t$-walk and thus the minimum odd $s t$-walk. Otherwise, $P_{\text {even }} \cup C_{\text {odd }}$ is not an odd $s t$-walk. Then $P_{\text {even }}$ 
and $C_{\text {odd }}$ must share at least 2 vertices and so $P_{\text {even }} \cup C_{\text {odd }}$ properly contains some odd stpath $P^{\prime}$. Since $w\left(P^{\prime}\right)<w\left(P_{\text {even }} \cup C_{\text {odd }}\right)$, the minimum odd $s t$-walk must be an odd $s t$-path, a contradiction. Thus, we can find a minimum weight odd $s t$-walk in polynomial time by finding $P_{\text {odd }}, P_{\text {even }}$ and $C_{\text {odd }}$ (in polynomial time as per Theorem 4.4.1) and choosing $P_{\text {odd }}$ if $w\left(P_{\text {odd }}\right) \leq w\left(P_{\text {even }} \cup C_{\text {odd }}\right)$ and choosing $P_{\text {even }} \cup C_{\text {odd }}$ otherwise.

\subsubsection{The Separation Problem for $s t-T$-Cuts}

Analogously to the analysis for odd-st-walks, it suffices to show that we can find a minimum weight st-T-cut in polynomial time. An early proof of this result is found in [16] and follows from a straightforward uncrossing argument; this argument is, in fact, known to work for a more general problem.

Let $G=(V, E)$ be a graph and let $T_{1}, T_{2} \subseteq V$ be such that $\left|T_{1}\right|$ and $\left|T_{2}\right|$ are even. Let $w \in \mathbb{R}_{+}^{E}$ be a weight function on the edges of $G$. A $T_{1}, T_{2}$-cut is a set of edges of the form $\delta(U)$ where $\left|U \cap T_{1}\right|$ and $\left|U \cap T_{2}\right|$ are odd. Note that choosing $T_{1}=T$ and $T_{2}=\{s, t\}$ in the below theorem implies we can find a minimum weight $s t-T$-cut in polynomial time.

Claim 4.4.3. Given graph $G=(V, E), T_{1}, T_{2} \subseteq V$ with $\left|T_{1}\right|,\left|T_{2}\right|$ even and $w \in \mathbb{R}_{+}^{E}$, there exists a polynomial time algorithm to find a minimum weight $T_{1}, T_{2}$-cut.

Proof. Recall that a $T$-cut is $\delta(U)$ such that $|U \cap T|$ is odd. It is well known that finding a minimum weight $T$-cut can be done in polynomial time [16].

First, find a minimum weight $T_{1}$-cut $\delta(U)$. If $\left|U \cap T_{2}\right|$ is odd, then $\delta(U)$ is a minimum $T_{1}, T_{2}$-cut. Otherwise, we require the following uncrossing property: "There exists a minimum weight $T_{1}, T_{2}$-cut $\delta(X)$ of $G$ such that $X \subseteq U$ or $X \subseteq \bar{U}$.".

Proof of uncrossing property:

Let $X$ be a minimum weight $T_{1}, T_{2}$-cut. If the uncrossing property does not hold, then $U$ and $X$ cross. This means $U \backslash X \neq \emptyset$ and $X \backslash U \neq \emptyset$.

Since $\left|X \cap T_{1}\right|$ is odd, $\left|X \cap U \cap T_{1}\right|$ and $\left|X \cap \bar{U} \cap T_{1}\right|$ have distinct parities. Since $\left|\bar{U} \cap T_{1}\right|$ is odd, $\left|X \cap \bar{U} \cap T_{1}\right|$ and $\left|\bar{X} \cap \bar{U} \cap T_{1}\right|$ have distinct parities. Thus $\left|X \cap U \cap T_{1}\right|$ and $\left|\bar{X} \cap \bar{U} \cap T_{1}\right|$ have the same parity. If they are both of odd parity, continue.

Otherwise, since $\left|U \cap T_{1}\right|$ is odd, $\left|X \cap U \cap T_{1}\right|$ and $\left|\bar{X} \cap U \cap T_{1}\right|$ have distinct parities. It follows that $\left|\bar{X} \cap U \cap T_{1}\right|$ and $\left|X \cap \bar{U} \cap T_{1}\right|$ are both of odd parity. If this is the case, since $\delta(X)$ and $\delta(\bar{X})$ are minimum weight $T_{1}, T_{2}$-cuts, we can exchange freely the roles of $X$ and $\bar{X}$ and thus assume that $\left|X \cap U \cap T_{1}\right|$ and $\left|\bar{X} \cap \bar{U} \cap T_{1}\right|$ both have odd parity. 
Since $\left|X \cap T_{2}\right|$ is odd, $\left|X \cap U \cap T_{2}\right|$ and $\left|X \cap \bar{U} \cap T_{2}\right|$ have distinct parities. Since $\left|\bar{U} \cap T_{2}\right|$ is even, $\left|X \cap \bar{U} \cap T_{2}\right|$ and $\left|\bar{X} \cap \bar{U} \cap T_{2}\right|$ have the same parity. Thus we may assume (after possibly interchanging both the role of $U$ and $\bar{U}$ and $X$ and $\bar{X}$ ) that $\left|X \cap U \cap T_{2}\right|$ is odd and $\left|\bar{X} \cap \bar{U} \cap T_{2}\right|$ is even. See Figure 4.1 for a representative figure of the necessary parities.

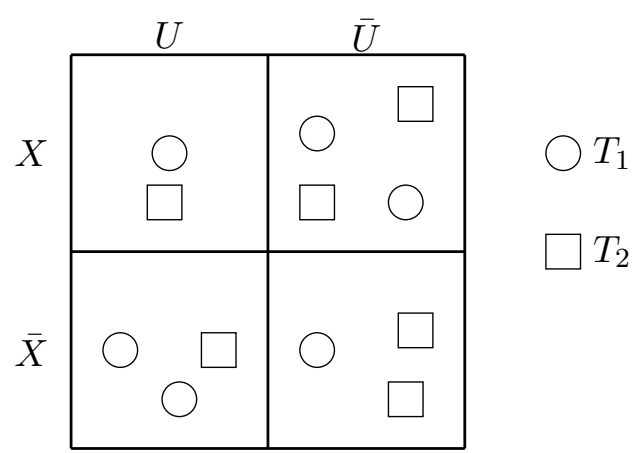

Figure 4.1: Uncrossing Illustration: $\left|X \cap U \cap T_{1}\right|$ is odd, etc.

It is well-known the weight function is submodular and thus

$$
w(\delta(X \cap U))+w(\delta(\bar{X} \cap \bar{U})) \leq w(\delta(U))+w(\delta(X)) .
$$

Since $\delta(U)$ is a minimum $T_{1}$-cut and $\bar{X} \cap \bar{U}$ is also a $T_{1}$-cut, $w(\delta(U)) \leq w(\delta(\bar{X} \cap \bar{U}))$. Since $\delta(X)$ is a minimum $T_{1}, T_{2}$-cut and $X \cap U$ is also a $T_{1}, T_{2}$-cut, $w(\delta(X)) \leq w(\delta(X \cap U))$. Thus equality must hold in (4.1). In particular $w(\delta(X))=w(\delta(X \cap U))$ and thus $X \cap U$ is a minimum $T_{1}, T_{2}$-cut of $G$ that is contained in $U$.

Returning to the main statement, if $U$ is not a $T_{1}, T_{2}$-cut, proceed recursively on the graphs $G_{1}$ and $G_{2}$ obtained by contracting respectively $U$ and $\bar{U}$ into a single $T_{1}$-vertex. The uncrossing lemma implies that a minimum $T_{1}, T_{2}$-cut in $G$ either corresponds to a minimum $T_{1}, T_{2}$-cut in $G_{1}$ or a minimum $T_{1}, T_{2}$-cut in $G_{2}$. Since $\left|V\left(G_{1}\right)\right|<|V(G)|$ and $\left|V\left(G_{2}\right)\right|<|V(G)|$, eventually the algorithm reaches a base case for which the minimum $T_{1}$-cut is also the minimum $T_{1}, T_{2}$-cut. Additionally, $\left|V\left(G_{1}\right)\right|+\left|V\left(G_{2}\right)\right|=|V(G)|+2$ and so the implied recursive algorithm runs in polynomial time.

Generalizations of separating $T_{1}, T_{2}$-cuts are interesting in their own right. In Section 5.2.1, these generalizations and related results are discussed. 


\subsection{Proof of Lemma 4.3.3}

\subsubsection{Preliminaries}

Let $\mathcal{F}$ be the clutter of odd $s t$-walks of signed graph $(G, \Sigma)$ with $s, t \in V(G)$. Up to isomorphism, there is exactly one signed graph whose set of odd $s t$-walks is exactly $\mathcal{O}_{K_{5}}$; odd- $K_{5}$ is the signed graph $\left(K_{5}, E\left(K_{5}\right)\right)$ where $K_{5}$ is the complete graph on five vertices and $s=t$ is any vertex. The correspondence between minors in clutters of odd-st-walks and minors in signed graphs (Proposition 4.2.1) shows that clutter $\mathcal{F}$ has an $\mathcal{O}_{K_{5}}$ minor if and only if $(G, \Sigma)$ has an odd- $K_{5}$ minor. Up to isomorphism, there is exactly one signed graph whose set of odd $s$-walks is exactly $\mathcal{L}_{7} ;\left(L_{7}, \Sigma_{7}\right)$ is the signed graph formed by taking the cycle on 4 vertices with all edges even and adding three odd edges each parallel to a distinct edge of the cycle. Vertices $s$ and $t$ are the endpoints of the edge with no parallel edge (see Figure 4.2). Again, Proposition 4.2.1 implies that clutter $\mathcal{F}$ has no $\mathcal{L}_{7}$ minor if and only if $(G, \Sigma)$ has no $\left(L_{7}, \Sigma_{7}\right)$ minor.

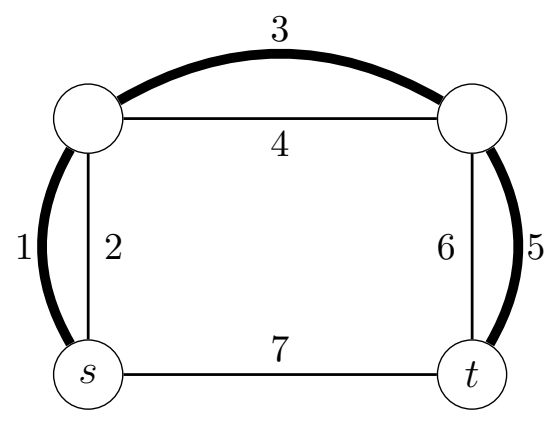

Figure 4.2: Signed graph $\left(L_{7}, \Sigma_{7}\right)$. Thick edges are odd.

To prove Lemma 4.3.3, it suffices to show that if $\mathcal{F}$ is Lehman, then we can find $I, J \subseteq E(\mathcal{F})$ such that $(G, \Sigma) / I \backslash J$ is odd- $K_{5}$ or $(G, \Sigma) / I \backslash J$ is $\left(L_{7}, \Sigma_{7}\right)$. Note that the odd- $K_{5}$ and $\left(L_{7}, \Sigma_{7}\right)$ we find must be such that the vertices labelled $s$ and $t$ are those vertices previously indicated.

Recall that an element in $b(\mathcal{F})$ is either an $s t$-bond of $G$ or a signature of $(G, \Sigma)$. By the hypothesis of the Lemma, we have the minimum cardinality sets $\overline{\mathcal{F}}$ of $\mathcal{F}$. We can construct clutter $\overline{\mathcal{K}}$ by solving equation (3.1). Hence throughout the proof, the clutter of minimum cardinality odd-st-walks and the clutter of minimum cardinality st-bonds and signatures are known. The proof has two parts: (1) when $s=t$ in $G$, we aim to find odd- $K_{5}$, and (2) when $s \neq t$ in $G$, we aim to find $\left(L_{7}, \Sigma_{7}\right)$. 


\subsubsection{Algorithm for Case 1: $s=t$ in $G$.}

As discussed in Section 2.4.2, $\mathcal{F}$ is the clutter of odd circuits of $(G, \Sigma)$ and $\mathcal{K}$ is the set of all inclusion-wise minimal signatures of $(G, \Sigma)$. To emphasize this difference, the elements of $\mathcal{F}$ will be denoted by $C$ and elements of $\mathcal{K}$ will be denoted by $B$ throughout this section.

A graph $H$ is a pseudo-odd- $K_{5}$ if there exist $e=x y \in E(H)$, a partition $\{x, y\}, V_{1}, V_{2}, V_{3}$ of $V(H)$ and paths $P_{1}, P_{2}, P_{3}, Q_{12}, Q_{13}, Q_{23}$ such that

i. $V_{1}, V_{2}, V_{3}$ are stable sets of $G$,

ii. $P_{1}, P_{2}, P_{3}$ are pairwise internally vertex disjoint,

iii. for all $i \in[3], P_{i}$ is between $x$ and $y$ and $V\left(P_{i}\right) \subseteq\{x, y\} \cup V_{i}$,

iv. for all $i, j \in[3], i \neq j, Q_{i j}$ joins $V\left(P_{i}\right)$ to $V\left(P_{j}\right)$, and $V\left(Q_{i j}\right) \subseteq V_{i} \cup V_{j}$.

We will proceed in two steps: first we show how to use $\overline{\mathcal{F}}$ and $\overline{\mathcal{K}}$ to find a pseudo-odd- $K_{5}$, then we show how to obtain an odd- $K_{5}$ from a pseudo-odd- $K_{5}$. The correctness essentially follows from the proof of Theorem 12 in [13] and will be given in Section 4.5.4.

\section{Finding a pseudo-odd- $K_{5}$.}

Pick an arbitrary edge $e=x y$ and choose $C_{1}, C_{2}, C_{3} \in \overline{\mathcal{F}}$ and $B_{1}, B_{2}, B_{3} \in \overline{\mathcal{K}}$ as in Lehman Corollary 3.4.6. For $i \in[3]$, let $P_{i}$ denote $C_{i}-e$; i.e. $P_{i}$ is a path between $x$ and $y$. Moreover, it can be shown that property (ii) holds for $P_{1}, P_{2}, P_{3}$. Since $B_{1}, B_{2}, B_{3}$ are signatures, $\Sigma^{\prime}=B_{1} \triangle B_{2} \triangle B_{3}$ is a signature of $(G, \Sigma)$. Contract all edges in $E(G)-\Sigma^{\prime}$ so that $E(G)=\Sigma^{\prime}$. For all $i, j \in[3], i \neq j, B_{i} \triangle B_{j}=\left(B_{i} \cup B_{j}\right)-e$ is a cut of $G$ since $B_{i}, B_{j}$ are signatures. Thus, we can find $U_{i j} \subseteq V(G)-\{x, y\}$ such that $\delta_{G}\left(U_{i j}\right)=B_{i} \triangle B_{j}$. For all distinct $i, j, k \in[3]$ we define $V_{i}$ as $U_{i j} \cap U_{i k}$. Then it can be shown that $\{x, y\}, V_{1}, V_{2}, V_{3}$ is a partition of $V(G)$ and that both properties (i) and (iii) hold. Moreover, it can also be shown that paths $Q_{12}, Q_{13}, Q_{23}$ satisfying property (iv) must exist. Thus $\left(G, \Sigma^{\prime}\right)$ is a pseudo-odd- $K_{5}$. Paths $Q_{i j}$ can be found efficiently as it is simply a matter of finding a path between two disjoint sets of vertices in the same component.

\section{Finding an odd- $K_{5}$}

It remains to show that given a pseudo-odd- $K_{5}$ we can either find a pseudo-odd- $K_{5}$ with fewer vertices, or we already have an odd- $K_{5}$. The argument follows the proof of Lemma 4.1 in [13]. First note that given a pseudo-odd- $K_{5}$ we may find $Q_{i j}$ such that only the endpoints intersect $P_{i}$ and $P_{j}$ (by possibly taking a subpath of the given path). Given a pseudo-odd- $K_{5}$, delete all edges outside of $P_{1} \cup P_{2} \cup P_{3} \cup Q_{12} \cup Q_{13} \cup Q_{23} \cup\{e\}$. Remove 
all degree two vertices $v$ by contracting edges of $\delta(v)$. The resulting graph is necessarily also a pseudo-odd- $K_{5}$. Moreover, each path $P_{i}$ contains exactly one internal vertex $v_{i}$ and since there are no degree two vertices $\left|V_{1}\right|=\left|V_{2}\right|=\left|V_{3}\right|$. We may assume $\left|V_{1}\right|>1$ for otherwise we already have an odd- $K_{5}$. For all distinct $i, j \in[3]$, let $e_{i j}$ be the edge of $Q_{i j}$ that is incident with $v_{i}$, let $Q_{i j}^{\prime}=Q_{i j}-\left\{e_{i j}, e_{j i}\right\}$, and let $V_{i}^{\prime}=V\left(Q_{i j}^{\prime}\right) \cap V\left(Q_{i k}^{\prime}\right)$. Define $G^{\prime}=G \backslash\left\{e_{13}, e_{32}, e_{21}\right\} /\left\{e_{12}, e_{23}, e_{31}\right\}$. Necessarily $G^{\prime}$ is a pseudo-odd- $K_{5}$ with partition $\{x, y\}, V_{1}^{\prime}, V_{2}^{\prime}, V_{3}^{\prime}$ and paths $P_{1}, P_{2}, P_{3}, Q_{12}^{\prime}, Q_{13}^{\prime}, Q_{23}^{\prime}$ with fewer vertices than the original pseudo-odd- $K_{5}$. Applying this reduction at most $\left|V_{1}\right|$ times, we find an odd- $K_{5}$.

\subsubsection{Algorithm for Case 2: $s \neq t$ in $G$.}

We look for an $\left(L_{7}, \Sigma_{7}\right)$ minor in $(G, \Sigma)$. The correctness essentially follows from the proof of Theorem 1.1 in [19] and will be given in Section 4.5.5.

It can be shown that there exists an edge $e=s t$ and vertex disjoint odd circuits $C_{s}, C_{t}$ such that $C_{s} \cup\{e\}, C_{t} \cup\{e\} \in \overline{\mathcal{F}}$ and $s \in V\left(C_{s}\right), t \in V\left(C_{t}\right)$. Moreover, there exists an odd circuit $C$ such that $C \cup\{e\} \in \overline{\mathcal{F}}$ and where $C$ and $C_{s}$ share exactly vertex $v_{s} \neq s$ and where $C$ and $C_{t}$ share exactly vertex $v_{t} \neq t$. It is clear that given $\overline{\mathcal{F}}$, we can find $C_{s}, C_{t}$ and $C$ as described above. Deleting all the edges outside of $C_{s} \cup C_{t} \cup C \cup\{e\}$ and shortening $C_{s}$, $C_{t}$ and $C$ to two edges by contracting all edges that do not have both ends in $\left\{s, t, v_{s}, v_{t}\right\}$ yields $\left(L_{7}, \Sigma_{7}\right)$.

\subsubsection{Proof for Case 1: $s=t$ in $G$}

This proof follows the proof in [28] with the exception that the contraction step occurs earlier.

\section{Finding a pseudo-odd- $K_{5}$}

We first show that any two minimum cardinality odd circuits of $(G, \Sigma)$ intersecting in one edge intersect exactly in the edge and its endpoints.

Claim 4.5.1. Let $C_{1}, C_{2} \in \overline{\mathcal{F}}$. If $e=x y \in C_{1} \cap C_{2}$, then $V\left(C_{1}\right) \cap V\left(C_{2}\right)=\{x, y\}$.

Proof. By way of contradiction, suppose there exists $v \in V\left(C_{1}\right) \cap V\left(C_{2}\right)-\{x, y\}$. Then there exists $P \subseteq C_{1} \cup C_{2}$ which is neither $C_{1}-e$ or $C_{2}-e$. By Lehman Corollary 3.4.7, $C_{1} \cup C_{2}-e$ contains no odd circuit. It follows that $P$ must have odd parity. However, then 
$P \cup e$ is an odd circuit in $C_{1} \cup C_{2}$ that is distinct from $C_{1}$ and $C_{2}$, contradicting Lehman Corollary 3.4.7.

As in the algorithm, pick an arbitrary edge $e=x y$ and find $C_{1}, C_{2}, C_{3} \in \overline{\mathcal{F}}$ and $B_{1}$, $B_{2}, B_{3} \in \overline{\mathcal{K}}$ using Lehman Corollary 3.4.6. For the remainder of this analysis, $i, j, k$ denote distinct indices from $\{1,2,3\}$.

By Claim 4.5.1, if $P_{i}=C_{i}-e$, then $P_{1}, P_{2}$ and $P_{3}$ are pairwise internally vertex disjoint paths; property (ii) holds as claimed. Since $B_{1}, B_{2}$ and $B_{3}$ are signatures, $B_{2} \triangle B_{3}$ is a cut and thus $B_{1} \triangle B_{2} \triangle B_{3}=B_{1} \cup B_{2} \cup B_{3}$ is a signature of $\left(G, \Sigma^{\prime}\right)$. Let $\left(G^{\prime}, \Sigma^{\prime}\right)$ be obtained from $G$ by contracting all the edges in $E(G)-\Sigma^{\prime}$ (this is allowed since $E(G)-\Sigma^{\prime}$ contains no odd circuit as $\Sigma^{\prime}$ is a signature). Note that $\left(G^{\prime}, \Sigma^{\prime}\right)$ is $\left(G^{\prime}, E\left(G^{\prime}\right)\right)$ and any signature of $\left(G^{\prime}, \Sigma^{\prime}\right)$ is also a signature of $(G, \Sigma)$.

Necessarily $B_{1}, B_{2}$ and $B_{3}$ are signatures of $\left(G^{\prime}, \Sigma^{\prime}\right)$ and so $B_{i} \triangle B_{j}=B_{i} \cup B_{j}-e$ is a cut of $G^{\prime}$; there exists $U_{i j} \subseteq V\left(G^{\prime}\right)-\{x, y\}$ such that $\delta_{G^{\prime}}\left(U_{i j}\right)=B_{i} \triangle B_{j}$. The edges of $E\left(G^{\prime}\right)-e$ are spanned by $\delta\left(U_{12}\right) \cup \delta\left(U_{13}\right) \cup \delta\left(U_{23}\right)$. Furthermore $U_{i j}$ necessarily induces a connected subgraph of $G$. Otherwise, there exists $\emptyset \subset T \subset U_{i j}$ with $\delta(T) \subset \delta\left(U_{i j}\right)$. Then $\delta(T) \triangle B_{j} \subseteq B_{i} \triangle B_{j}$ is a signature of $G^{\prime}$ and thus a signature of $G$ contained in $B_{i} \cup B_{j}$ that is neither $B_{i}$ nor $B_{j}$, contradicting Lehman Corollary 3.4.7. Given $B_{i}, B_{j}$, we can find $\overline{U_{i j}}$ efficiently using breath first search in $G \backslash\left(B_{i} \triangle B_{j}\right)$ starting from $x$.

Now, $\delta\left(U_{12} \triangle U_{23} \triangle U_{13}\right)=\emptyset$. Since $G$ is connected, $U_{12} \triangle U_{23} \triangle U_{13}$ must be $\emptyset$ since $x, y \notin U_{i j}$. So $U_{i j}=V_{i} \cup V_{j}$ where $V_{1}, V_{2}, V_{3}$ are pairwise disjoint sets of $V \backslash\{x, y\}$. It is clear that $E\left(G^{\prime}\right)-e$ is spanned by $\delta\left(V_{1} \cup V_{2}\right) \cup \delta\left(V_{1} \cup V_{3}\right) \cup \delta\left(V_{2} \cup V_{3}\right)$ and $V\left(G^{\prime}\right)-\{x, y\}$ is spanned by $V_{1} \cup V_{2} \cup V_{3}$. In other words, $V_{1}, V_{2}, V_{3}$ are independent sets spanning $V \backslash\{x, y\}$. So, we have shown property (i) holds.

Since $E_{G}\left(P_{i}\right)$ does not contain any edge of $B_{j} \triangle B_{k}$, the vertices of $P_{i}$ are disjoint from $V_{j} \cup V_{k}$ in $G^{\prime}$. Since $\left|P_{i} \cap B_{i}\right| \geq 3, P_{i}$ must be a length 2 path between $x$ and $y$ with internal vertex $v_{i} \in V_{i}$. So, we have shown properties (ii) and (iii) hold. Lastly, since $U_{i j}$ induces a connected subgraph of $G^{\prime}$, there must exist a paths $Q_{i j}$ satisfying property (iv).

\section{Finding an odd- $K_{5}$}

Given a pseudo-odd- $K_{5}(G, E(G))$, delete all edges outside of $P_{1} \cup P_{2} \cup P_{3} \cup Q_{12} \cup Q_{13} \cup Q_{23} \cup e$ and remove all degree two vertices by contracting $\delta(v)$. Necessarily $V_{1}=V\left(Q_{12}\right) \cap V\left(Q_{13}\right)$, $V_{2}=V\left(Q_{12}\right) \cap V\left(Q_{23}\right), V_{3}=V\left(Q_{23}\right) \cap V\left(Q_{13}\right)$ and so $\left|V_{1}\right|=\left|V_{2}\right|=\left|V_{3}\right|$. If $\left|V_{1}\right|=1$, we have found an odd $K_{5}$ minor of $(G, \Sigma)$ because $(G, \Sigma)$ is isomorphic to odd $-K_{5}$. 
Otherwise, for distinct $\{i, j\} \in\{1,2,3\}$ let $e_{i j}$ be the edge on $Q_{i j}$ incident with $v_{i}$. Let $G^{\prime}=G /\left\{e_{12}, e_{23}, e_{3}\right\} \backslash\left\{e_{13}, e_{32}, e_{21}\right\}, P_{i}^{\prime}=P_{i}, Q_{i j}^{\prime}=Q_{i j} \backslash\left\{e_{i j}, e_{j i}\right\}, V_{i}^{\prime}=V\left(Q_{i j}^{\prime}\right) \cap V\left(Q_{i j}^{\prime}\right)$, and $V_{0}^{\prime}=\{x, y\}$. It can be verified that $\left(G^{\prime}, E\left(G^{\prime}\right)\right)$ is a minor of $(G, E(G))$ that is also a pseudo-odd- $K_{5}$. Additionally, $\left|V_{1}^{\prime}\right|<\left|V_{1}\right|$. Continue inductively to find the odd- $K_{5}$ minor. Since the inductive step is applied $\left|V_{1}\right|<n$ times and all the graph operations are implementable in polynomial time, odd $K_{5}$ can be found in polynomial time.

\subsubsection{Proof for Case 2: $s \neq t$ in $G$}

This argument follows the proof in [19].

Call an odd circuit $C$ minimum if there exists an even path $P$ such that $C \cup P \in \overline{\mathcal{F}}$. Analogously, call an even st-path $P$ minimum if there exists an odd circuit $C$ such that $C \cup P \in \overline{\mathcal{F}}$. Call an odd st-path $P$ minimum if $P \in \overline{\mathcal{F}}$. To prove correctness of the algorithm, it suffices to show the existence of $C_{s}, C_{t}$ and $C$ as described by the algorithm.

Claim 4.5.2. There exists a minimum odd circuit $C_{s}$ containing $s$ and a minimum odd circuit $C_{t}$ containing $t$. Furthermore, any minimum odd circuit containing $s$ and any minimum odd circuit containing $t$ must be vertex disjoint.

Proof. Since $\delta(s)$ is an st-cut, it contains some $K \in \overline{\mathcal{K}}$ since it necessarily intersects every (odd or even) st-path. Accordingly, there exists $B \subseteq \delta(s)$ such that $|B \cap F| \geq 1$ for all $F \in \overline{\mathcal{F}}$. By Lehman Corollary 3.4.5, $B$ intersects some $F \in \overline{\mathcal{F}}$ at least twice. This $F$ cannot be an odd st-path and thus it must be the union of an even st-path and an odd circuit. Thus there exists a minimum odd circuit $C_{s}$ using $s$. Analogously, there exists a minimum odd circuit $C_{t}$ using $t$.

Let $C_{s}$ be any minimum odd circuit containing $s$ and let $C_{t}$ be any minimum odd circuit containing $t$. Suppose $C_{s}$ uses $t$. Then $C_{s}$ can be partitioned into st-paths $P$ and $P^{\prime}$ one of which must be odd. This contradicts the fact $\mathcal{F}$ is a clutter. Thus $C_{s}$ cannot use $t$. Suppose $C_{s}$ uses some vertex $v$ on $C_{t}$ where $v \neq t$. Then $C_{s}$ can be partitioned into $s v$-paths $P_{s}$ and $P_{s}^{\prime}$. We may assume $P_{s}$ is odd and $P_{s}^{\prime}$ is even since $C_{s}$ is odd. Similarly, $C_{t}$ can be partitioned into $t v$-paths $P_{t}$ and $P_{t}^{\prime}$. We may assume $P_{t}$ is odd and $P_{t}^{\prime}$ is even. Then $P_{s} \cup P_{t}^{\prime}$ must contain an odd $s t$-walk $F$. Since $F$ cannot be the odd $s t$-walk corresponding to $C_{s}$ or the odd $s t$-walk corresponding to $C_{t}$, this is a contradiction to Lehman Corollary 3.4.7. Therefore $C_{s}$ and $C_{t}$ are vertex disjoint.

Henceforth $C_{s}$ and will be a fixed minimum odd circuit containing $s$ and $C_{t}$ will be a fixed minimum odd circuit containing $t$. 
Claim 4.5.3. There exists a unique minimum st-path $P_{\text {even }}$.

Proof. Let $P_{s}$ be the minimum even st-path such that $P_{s} \cup C_{s} \in \overline{\mathcal{F}}$. Let $P_{t}$ be the minimum even st-path such that $P_{t} \cup C_{t} \in \overline{\mathcal{F}}$. Then $P_{s}=P_{t}$ because otherwise $P_{s} \cup C_{t}$ contains an odd $s t$-walk that is neither $P_{s} \cup C_{s}$ or $P_{t} \cup C_{t}$, a contradiction. This analysis holds for any for $F=P \cup C \in \overline{\mathcal{F}}$ such that $C \neq C_{s}$.

Claim 4.5.4. Let $F \in \overline{\mathcal{F}}$ share vertex $v \neq s$ with $C_{s}$. Then either

1. $F$ is an odd st-path and uses an edge of $C_{s}$ incident to $s$, or,

2. $F$ is of the form $P_{\text {even }} \cup C$ where $C$ is a minimum odd circuit. Moreover, if $C$ shares another vertex $v^{\prime}$ with $C_{s}$ as well, $C$ and $C_{s}$ are not edge disjoint.

Proof. By Claim 4.5.3, $F$ either an odd st-path or of the form $P_{\text {even }} \cup C$ where $C$ is a minimum odd circuit.

Suppose $F$ is an odd-st-path. Partition circuit $C_{s}$ into $s v$-paths $Q$ (odd) and $Q^{\prime}$ (even). The vertex $v$ splits $F$ into a path $F_{1}$ from $s$ to $v$ and a path $F_{2}$ from $v$ to $t$; exactly one of $F_{1}$ and $F_{2}$ is even. By way of contradiction, suppose $F_{1}$ is distinct from $Q$ and $Q^{\prime}$. If $F_{2}$ is odd, $Q^{\prime} \cup F_{2}$ is an odd st-path contained in $F \cup C_{s}$ that is neither $F$ nor $C_{s} \cup P_{\text {even }}$, contradicting Lehman Corollary 3.4.7. If $F_{2}$ is even, $Q \cup F_{2}$ is an odd st-path contained in $F \cup C_{s}$ that is neither $F$ nor $C_{s} \cup P_{\text {even }}$, again contradicting Lehman Corollary 3.4.7. Thus $F_{1}$ is not distinct from $Q$ and $Q^{\prime}$ and so $F$ uses an edge of $C_{s}$ incident to $s$.

Suppose $F$ is of the form $P_{\text {even }} \cup C$ where $C$ is a minimum odd circuit. If $C$ additionally shares vertex $v^{\prime}$ with $C_{s}$, we must show $C$ and $C_{s}$ are not edge disjoint. By way of contradiction, suppose $C$ and $C_{s}$ are edge disjoint. Then $v$ and $v^{\prime}$ partition $C_{s}$ into $P$ (odd) and $P^{\prime}$ (even) and $v$ and $v^{\prime}$ partition $C$ into $Q$ (odd) and $Q^{\prime}$ (even). All of $P, P^{\prime}, Q, Q^{\prime}$ must be distinct since $C$ and $C_{s}$ are edge disjoint. Then $P_{\text {even }} \cup P \cup Q^{\prime}$ contains an odd st-walk distinct from $F$ and $P_{\text {even }} \cup C_{s}$, contradicting Lehman Corollary 3.4.7.

Assume $F_{1}=P_{\text {even }} \cup C_{s}$ and let $B_{1}$ denote the mate of $F_{1}$.

Claim 4.5.5. The edges in $F_{1} \cap B_{1}$ are the 2 edges of $C_{s}$ incident to $s$ and one edge of $P_{\text {even. }}$ Thus $\left|F_{1} \cap B_{1}\right|=q=3$.

Proof. By way of contradiction, suppose there exists $e \in\left(F_{1} \cap B_{1}\right) \backslash \delta(s)$. By Lehman Corollary 3.4.3, there exists $F_{2} \in \overline{\mathcal{F}}$ such that $F_{1} \cap F_{2}=\{e\}$. By Claim 4.5.3, $F_{2}$ is an st-path because since otherwise $F_{1}$ and $F_{2}$ share both $e$ and $P_{\text {even }}$. By Claim 4.5.4, since $F_{2}$ is an st-path it must use an edge of $C_{s}$ incident to $s$. However, $e$ is not incident with $s$ and thus we have a contradiction. We conclude $F_{1} \cap B_{1} \subseteq \delta(s) \cup P_{\text {even }}$ and, moreover, since $\mathcal{F}$ is binary and $\left|B_{1} \cap\left(P_{\text {even }} \cup C_{t}\right)\right|=1,\left|F_{1} \cap B_{1}\right|=3$ and $\left|B_{1} \cap P_{\text {even }}\right|=1$. 
Let $e_{s} \in B_{1} \cap P_{\text {even }}$ and obtain $\left\{F_{1}^{\prime} \ldots F_{\ell}^{\prime}\right\} \in \overline{\mathcal{F}}$ intersecting in at most $e_{s}$ using Lehman Corollary 3.4.1 where $F_{1}^{\prime}=F_{1}$. Note that there exists $v \in C_{s}$ since $C_{s}$ is not a loop by Claim 4.5.5.

Claim 4.5.6. Let $v \neq s \in V\left(C_{s}\right)$. Then there exists $F^{\prime} \in\left\{F_{2}^{\prime}, \ldots F_{\ell}^{\prime}\right\}$ of the form $P_{\text {even }} \cup C$ where $v \in V(C)$.

Proof. By Claim 4.5.4, no odd-st-path in $\left\{F_{2}^{\prime}, \ldots F_{\ell}^{\prime}\right\}$ uses an edge in $\delta(v)$ since otherwise such a path would also intersect $F_{1}$ twice: in $e_{s}$ and in some edge $e \in \delta(s) \backslash e_{s}$. If there does not exist $F^{\prime}$ of the form $P_{\text {even }} \cup C$ with $v \in V(C)$, since $E(G)=F_{1}^{\prime} \cup \ldots \cup F_{\ell}^{\prime}$, by Lehman Corollary 3.4.5, $v$ must have degree exactly 2. If $\delta(v)=\left\{e, e^{\prime}\right\}$, every odd $s t$-walk uses both or none of $e, e^{\prime}$ and so the columns of $M(\overline{\mathcal{F}})$ indexed by $e, e^{\prime}$ are identical. This contradicts the fact $M(\overline{\mathcal{F}})$ is nonsingular. Thus there exists $F^{\prime}$ of the form $P_{\text {even }} \cup C$ where $C$ uses $v$.

Claim 4.5.7. $P_{\text {even }}$ consists of a single edge $e_{s}$.

Proof. If $\left|P_{\text {even }}\right| \geq 2$, all sets of $\left\{F_{2}^{\prime}, \ldots F_{\ell}^{\prime}\right\}$ are odd st-paths, contradicting Claim 4.5.6.

Now, odd st-walk $F^{\prime}$ is in $\left\{F_{2}^{\prime}, \ldots F_{\ell}^{\prime}\right\}$ if and only if $e_{s}$ is contained in both $F^{\prime}$ and its mate. By Claim 4.5.5, there are exactly 3 such sets: $C_{s} \cup e_{s}, C_{t} \cup e_{t}$ and $C \cup e_{t}$ for some other odd circuit $C$. Since necessarily $C_{s}$ and $C_{t}$ are vertex disjoint, for any vertex $v_{s} \in V\left(C_{s}\right)$ and $v_{t} \in V\left(C_{t}\right)$ it must be that $v_{s}, v_{t} \in C$ by Claim 4.5.6. Therefore, we showed there exist vertex disjoint odd circuits $C_{s}, C_{t}$ and $e=s t$ such that $C_{s} \cup\{e\}$ and $C_{t} \cup\{e\} \in \overline{\mathcal{F}}$ and where $s \in V\left(C_{s}\right), t \in V\left(C_{t}\right)$. Moreover, $C$ is such that $C \cup\{e\} \in \overline{\mathcal{F}}$ and $C$ and $C_{s}$ share exactly one vertex $v_{s} \neq s$ and $C$ and $C_{t}$ share exactly one vertex $v_{t} \neq t$. This concludes the proof of correctness for the given algorithm.

\subsection{Proof of Lemma 4.3.5}

Even though this algorithm is essentially a constructive proof of the version in [19], it was necessary to change the organization of the proof and so the below exposition differs from that in the original paper. 


\subsubsection{Preliminaries}

Let $\mathcal{F}$ be the clutter of $s t$ - $T$-cuts of $(G, T)$ with $s, t \in V(G)$. Up to isomorphism, there is exactly one graft whose set of $s t$-T-cuts is exactly $b\left(\mathcal{O}_{K_{5}}\right) ;\left(O_{5}, T_{5}\right)$ is the graft in Figure 4.3. The correspondence between minors in clutters of $s t-T$-cuts and minors in grafts (Proposition 4.2.2) shows that clutter $\mathcal{F}$ has a $b\left(\mathcal{O}_{K_{5}}\right)$ minor if and only if $(G, T)$ has a $\left(O_{5}, T_{5}\right)$ minor. Up to isomorphism, there are exactly two grafts whose sets of $s t-T$-cuts are exactly $\mathcal{L}_{7} ;\left(L_{1}, T_{1}\right)$ and $\left(L_{2}, T_{2}\right)$ are the grafts in Figure 4.4. Again, Proposition 4.2.2 implies that clutter $\mathcal{F}$ has no $\mathcal{L}_{7}$ minor if and only if $(G, T)$ has no $\left(L_{1}, T_{1}\right)$ or $\left(L_{2}, T_{2}\right)$ minor.

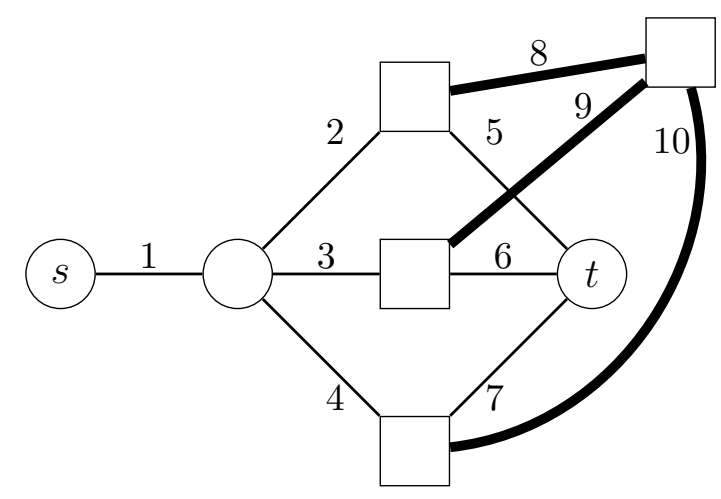

Figure 4.3: Graft $\left(O_{5}, T_{5}\right)$. Square vertices are in $T$.
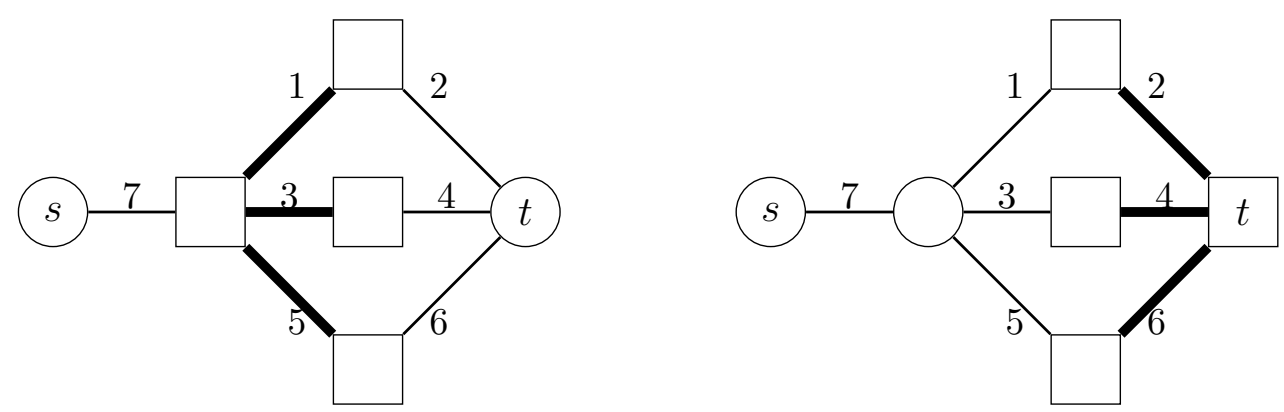

Figure 4.4: Grafts $\left(L_{1}, T_{1}\right)$ (left) and $\left(L_{2}, T_{2}\right)$ (right). Square vertices are in $T$.

To prove Lemma 4.3.5, it suffices to show that if $\mathcal{F}$ is Lehman, we can find $I, J \subseteq E(\mathcal{F})$ such that $(G, T) / J \backslash I$ is $\left(O_{5}, T_{5}\right),\left(L_{1}, T_{1}\right)$ or $\left(L_{2}, T_{2}\right)$. 
Recall that an element in $b(\mathcal{F})$ is either an st-path or a $T$-join (see Section 2.4.5). By the hypothesis of the Lemma, we have the minimum cardinality sets $\overline{\mathcal{F}}$ of $\mathcal{F}$. We can construct clutter $\overline{\mathcal{K}}$ by solving equation (3.1). Hence throughout the proof, the clutter of minimum cardinality odd-st-walks and the clutter of minimum cardinality st-bonds and signatures are both known.

We will refer to elements of $\overline{\mathcal{K}}$ as minimum $T$-joins and minimum st-paths. A 3-path configuration is a set of st-paths $P_{1}, P_{2}, P_{3}$ such that

i. $s \notin T$;

ii. there exists edge $s s^{\prime} \in P_{1} \cap P_{2} \cap P_{3}$;

iii. the only vertices common to more than one of $P_{1}, P_{2}$ and $P_{3}$ are $s, s^{\prime}$ and $t$; and

iv. each path $P_{i}$ contains a vertex $v_{i} \in T$ distinct from $s, s^{\prime}$ and $t$.

We define a link of $(G, T)$ as a path with both endpoints in $V\left(P_{1}\right)-s, V\left(P_{2}\right)-s, V\left(P_{3}\right)-s$ and no internal vertices in $P_{1}, P_{2}$ or $P_{3}$. We call a link odd if is has an odd number of internal vertices in $T$. We call a link even if it has an even number of internal vertices in $T$.

The algorithm has two main steps. The first is to find minimum st-paths $P_{1}, P_{2}$ and $P_{3}$ forming a 3-path configuration. If all $T$ vertices appear on these paths, we are done; delete all edges except $P_{1} \cup P_{2} \cup P_{3}$ and contract all edges except $s s^{\prime}, \delta\left(v_{1}\right), \delta\left(v_{2}\right)$ and $\delta\left(v_{3}\right)$. Otherwise, perform contractions and deletions maintaining the 3 -path configuration to either push all the $T$ vertices to the paths or find $\left(O_{5}, T_{5}\right)$.

Recall that $r$ denotes the cardinality of sets in $\overline{\mathcal{F}}$ (the set of minimum $s t$ - $T$-cuts) and $\ell$ denotes the cardinality of sets in $\overline{\mathcal{K}}$ (the set of minimum st-paths and minimum $T$-joins). We break the algorithm into two cases: $\ell \geq 4$ and $\ell=3$. Much of the analysis carries through in both cases. For $\ell=3$, st-paths in the 3-path configuration do not have an edge incident with any of $\left\{s, s^{\prime}, t\right\}$ and so we must reason directly about the structure after some common preliminaries.

\subsubsection{Algorithm for Case 1: $\ell \geq 4$}

Finding $P_{1}, P_{2}$ and $P_{3}$.

Choose $e=s s^{\prime}$ such that $s \notin T$ and $s^{\prime} \neq t$. If no such $e$ exists, switch the role of $s$ and $t$ and we are guaranteed that such an edge exists. Use Lehman Corollary 3.4.6 to find minimum paths $P_{1}, P_{2}, P_{3}$ intersecting exactly in $e$ whose mates $B_{1}, B_{2}, B_{3}$ intersect exactly in $e$. If $r=3$, then all the $T$ vertices are located on the 3 -path configuration $P_{1}, P_{2}, P_{3}$; if $v_{i}$ is a 
$T$ vertex of $P_{i}$, deleting all edges except $P_{1} \cup P_{2} \cup P_{3}$ and contracting all remaining edges except $s s^{\prime}$ and $\delta\left(v_{i}\right)$ for $i=1,2,3$, yields either $\left(L_{1}, T_{1}\right)$ or $\left(L_{2}, T_{2}\right)$.

\section{Pushing $T$ vertices to paths.}

Let edge $w w^{\prime} \in P_{1}-\left(\delta(s) \cup \delta\left(s^{\prime}\right) \cup \delta(t)\right)$; such edge exists since $r \geq 4$. Using Lehman Corollary 3.4.6, find minimum $T$-joins $K_{1}, K_{2}$ intersecting in exactly $w w^{\prime}$ whose mates intersect in exactly $w w^{\prime}$. Then $C=K_{1} \triangle K_{2}$ is a circuit. If both $s^{\prime}, t \in V(C)$, then all the $T$ vertices are located on the 3-path configuration $P_{1}, P_{2}, P_{3}$ and we are done. Otherwise, either $s^{\prime} \notin T$ or $t \notin T$.

Partition edges of $C-\left(P_{1} \cup P_{2} \cup P_{3}\right)$ into a collection $\mathcal{S}$ of links. Vertices of $T$ are necessarily either on a link of $\mathcal{S}$ or in $P_{1} \cup P_{2} \cup P_{3}$. For every $Q \in \mathcal{S}$, contract all edges of $Q$ except for the two edges incident to the ends of $Q$. The parity of $Q$ remains unchanged; if $Q$ is odd then the internal vertex of $Q$ is in $T$ and if $Q$ is even then the internal vertex of $Q$ is outside $T$. Update $\mathcal{S}$ by removing all even links. For any pair $S_{1}, S_{2} \in \mathcal{S}$ sharing endpoint $v$, contract edge of $S_{1}, S_{2}$ incident to $v$ and remove $S_{1}, S_{2}$ from $\mathcal{S}$. If link $S \in \mathcal{S}$ has endpoint $v \in V\left(P_{i}\right)-\left\{s, s^{\prime}, t\right\}$ such that $v \notin T$ or there exists $v^{\prime} \in T-\left\{s^{\prime}, t, v\right\}$ in $V\left(P_{i}\right)$, then contract the edge of $S$ incident to $v$ and update $\mathcal{S}$ by removing $S$.

If after applying this procedure, $\mathcal{S}=\emptyset$, then all the $T$ vertices are located on the 3-path configuration $P_{1}, P_{2}, P_{3}$ and as before find $\left(L_{1}, T_{1}\right)$ or $\left(L_{2}, T_{2}\right)$. Otherwise $\mathcal{S}$ consists of a

single odd link with $T$ vertex $y$. Either there exists paths $Q_{1}, Q_{2}, Q_{3}$ from $y$ to each of $v_{1}, v_{2}, v_{3}$ that use no vertex of $P_{1} \cup P_{2} \cup P_{3}$ or there exists a path $Q$ from $v$ to $s^{\prime}$ or $t$. In the first case, deleting all edges except $P_{1} \cup P_{2} \cup P_{3}$ and contracting all remaining edges except $s s^{\prime}, \delta\left(v_{i}\right)$ for $i=1,2,3$ and $\delta(y)$ yields $\left(O_{5}, T_{5}\right)$. In the second case, deleting all edges except $P_{1} \cup P_{2} \cup P_{3} \cup Q$ and contracting all remaining edges except $s s^{\prime}$ and $\delta\left(v_{i}\right)$ for $i=1,2,3$ yields $\left(L_{1}, T_{1}\right)$ or $\left(L_{2}, T_{2}\right)$.

\subsubsection{Proof of Correctness for Case 1: $\ell \geq 4$}

Finding $P_{1}, P_{2}$ and $P_{3}$.

This proof essentially follows from the proof of Theorem 1.3 in [19]; specifically, we require the following claims. Proofs are included for completeness.

Claim 4.6.1. Let $K_{1}, K_{2} \in \mathcal{K}$ be two minimum $T$-joins. Then $K_{1} \triangle K_{2}$ is a circuit that does not use both $s$ and $t$. 
Proof. Since $K_{1}$ and $K_{2}$ are $T$-joins, the vertices of $G\left[K_{1} \triangle K_{2}\right]$ have even degree. Thus $K_{1} \triangle K_{2}$ can be expressed as the disjoint union of some set of cycles $\mathcal{C}$ of $G$. Let $C \in \mathcal{C}$. Then $K_{1} \triangle C$ contains a $T$-join and by Lehman Corollary 3.4.7, $K_{1} \triangle C$ must be either $K_{1}$ or $K_{2}$. Necessarily $K_{1} \triangle C \neq K_{1}$ and so $K_{1} \triangle C=K_{2}$, or equivalently, $K_{1} \triangle K_{2}=C$. Suppose $C$ contains both $s$ and $t$. Since $K_{1}$ and $K_{2}$ intersect in exactly one edge, $\left|K_{1} \triangle K_{2}\right|=2 \ell-2$ and thus $C$ contains an st-path of cardinality at most $\ell-1$. This contradicts the fact the minimum cardinality elements of $\overline{\mathcal{K}}$ have size $\ell$. Therefore, $K_{1} \triangle K_{2}$ is a circuit that does not use both $s$ and $t$.

If $P$ is a path and $x, y \in V(P)$, let $P[x, y]$ denotes the subpath of $P$ between $x$ and $y$.

Claim 4.6.2. Let $K_{1}, K_{2} \in \mathcal{K}$ be two minimum st-paths. Then if $v \in V\left(K_{1}\right) \cap V\left(K_{2}\right)$, $K_{1}[s, v]=K_{2}[s, v]$ or $K_{1}[v, t]=K_{2}[v, t]$. In particular, if $K_{1}$ and $K_{2}$ intersect in exactly $e$, then $e \in \delta(s) \cup \delta(t)$.

Proof. The set $K_{1}[s, v] \cup K_{2}[v, t]$ contains an st-path. Lehman Corollary 3.4.7 implies that this path is either $K_{1}$ or $K_{2}$. The result follows.

Claim 4.6.3. Let $e \in E-\delta(s)-\delta(t)$. Then there exist two minimum $T$-joins $K_{1}$ and $K_{2}$ intersecting exactly in e. Endpoints of e that are not in circuit $C=K_{1} \triangle K_{2}$ are in $T$. At most one endpoint of $e$ is a vertex of $C$ and thus at least one endpoint of $e$ is in $T$.

Proof. By Lehman Corollary 3.4.6, there exist $K_{1}, K_{2}, K_{3} \in \bar{K}$ intersecting exactly in $e$. Applying Claim 4.6.2, we may assume $K_{1}$ and $K_{2}$ are $T$-joins. Let $e=x y$, If $x \notin C$, then $K_{1} \triangle \delta(x)=\{e\}$ and thus $x \in T$. If $x, y \in C$, by Lehman Corollary 3.4.6, there exist st-T-cuts $F_{1}, F_{2}, F_{3} \in \overline{\mathcal{F}}$ intersecting in $e$. Since $F_{1}, F_{2}, F_{3}$ are cuts and $x, y \in C$, it must be that $\left|C \cap F_{i}\right| \geq 2$ for all $i \in[3]$. It follows that each of $F_{1}, F_{2}, F_{3}$ intersect at least one of $K_{1}, K_{2}$ twice. This is a contradiction since at most two of $F_{1}, F_{2}$ can be mates of $K_{1}, K_{2}$.

Claim 4.6.4. No minimum $T$-join intersects both $\delta(s)$ and $\delta(t)$.

Proof. Suppose $K \in \overline{\mathcal{K}}$ contains an edge incident to $s$ and an edge incident to $t$. Let $F$ be the mate of $K$. Suppose there exists $e \in(K \cap F)-\delta(s)-\delta(t)$. By Lehman Corollary 3.4.6, there exists $K_{1}, K_{2} \in \overline{\mathcal{K}}$ such that $K, K_{1}$ and $K_{2}$ intersect exactly in e. By Claim 4.6.3, we may assume $K_{1}$ is a minimum $T$-join. Then $K \triangle K_{2}$ uses both $s$ and $t$, contradicting Claim 4.6.1. Since $|J \cap U| \geq 3$, we may assume $\left\{e_{s}, e_{s}^{\prime}, e_{t}\right\} \subseteq J \cap U$ for $e_{s}, e_{s}^{\prime} \in \delta(s)$ and $e_{t} \in \delta(t)$. Since $r \geq 3$, there exists $e \in E-\delta(s)-\delta(t)$. Minimum $T$-joins $K_{1}^{\prime}, K_{2}^{\prime}$ intersecting in exactly $e$ must exist and so there exists minimum $T$-join $K_{1}^{\prime}$ that does not contain $e_{t}$. By 
Claim 4.6.1, $K \triangle K_{1}^{\prime}$ is a circuit using one of $s$ and $t$; by construction, $K \triangle K_{1}^{\prime}$ uses $t$ but not $s$. Since $K_{1}$ is not the mate of $F$, it does not contain both $e_{s}$ and $e_{s}^{\prime}$. This contradicts the fact $K \triangle K_{1}$ does not use $s$.

Claim 4.6.5. Minimum T-joins are either disjoint from $\delta(s)$ or disjoint from $\delta(t)$.

Proof. Suppose $K_{s}, K_{t} \in \overline{\mathcal{K}}$ are such that $K_{s} \cap \delta(s) \neq \emptyset$ and $K_{t} \cap \delta(t) \neq \emptyset$. By Claim 4.6.4 $K_{s} \cap \delta(t)=\emptyset$ and $K_{t} \cap \delta(s)=\emptyset$. Thus $K_{s} \triangle K_{t}$ is a circuit intersecting both $s$ and $t$, a contradiction to Claim 4.6.1.

Claim 4.6.6. We can find a 3-path configuration.

Proof. Suppose minimum $T$-joins are disjoint from $\delta(s)$ and thus $s \notin T$. Let $s s^{\prime} \in \delta(s)$. By Lehman Corollary 3.4.6, there exist $K_{1}, K_{2}, K_{3} \in \overline{\mathcal{K}}$ intersecting in exactly $s s^{\prime}$. Since $s \notin T, K_{1}, K_{2}, K_{3}$ must be st-paths. By Claim 4.6.3, $K_{1}, K_{2}, K_{3}$ pairwise share only vertices $s, s^{\prime}, t$. For each path $K_{i}$, there exists $e_{i}=x_{i} y_{i}$ such that $x_{i}, y_{i} \notin\left\{s, s^{\prime}, t\right\}$ since $K_{i}$ has length at least 4. By Claim 4.6.3, at least one of $x_{i}, y_{i}$ is in $T$. It follows that $K_{1}, K_{2}, K_{3}$ is a 3 -path configuration.

In summary, this section shows we can find a 3-path configuration by simply choosing $s \notin T$ (swapping roles of $s$ and $t$ if necessary), choosing arbitrary $e \in \delta(s)$ and using Lehman Corollary 3.4.6 to obtain 3 st-paths intersecting in exactly $e$. Necessarily minimum $T$-joins may be assumed disjoint from $\delta(s)$.

\section{Pushing $T$ vertices to paths.}

The proof in this section is, in spirit, the same as the corresponding proof in [19]. However, we must replace a minimality assumption with a method for explicitly finding the minimal instance.

Claim 4.6.7. Let $(G, T)$ be a graft that contains a 3-path configuration $P_{1}, P_{2}, P_{3}$. If all vertices of $T$ are in $P_{1} \cup P_{2} \cup P_{3}$, then $(G, T)$ contains a $\left(L_{1}, T_{1}\right)$ or $\left(L_{2}, T_{2}\right)$ minor.

Proof. For $i \in[3]$, contract all edges of $P_{i}$ except $\delta\left(v_{i}\right)$ where $v_{i} \in T$. Delete all edges outside $P_{1} \cup P_{2} \cup P_{3}$. In the resulting graft, necessarily one of $s^{\prime}$ and $t$ is a $T$-vertex and so we have either $\left(L_{1}, T_{1}\right)$ or $\left(L_{2}, T_{2}\right)$.

Claim 4.6.8. If $r=3$, then all vertices of $T$ are in $P_{1} \cup P_{2} \cup P_{3}$. 
Proof. By Lehman Corollary 3.4.2, $E=P_{1} \cup P_{2} \cup P_{3}$ and so the result follows.

Let edge $w w^{\prime} \in P-\delta(s)-\delta\left(s^{\prime}\right)-\delta(t)$. Such an edge must exist since $r \geq 4$. By Lehman Corollary 3.4.4, there exists a collection $\mathcal{K}^{\prime} \subseteq \overline{\mathcal{K}}=\left\{K_{1}, K_{2} \ldots K_{r}\right\}$ intersecting in at most $w w^{\prime}$. Using Claim 4.6.3, we may assume $K_{1}, K_{2} \in \mathcal{K}$ intersect in exactly $w w^{\prime}$ and the mates of $K_{1}$ and $K_{2}$ also intersect in exactly $w w^{\prime}$. By Claim 4.6.5 $C=K_{1} \triangle K_{2}$ is a circuit that does not use $s$. In fact, we can do better.

Claim 4.6.9. If $C$ uses both $s^{\prime}$ and $t$, then the vertices of $T$ are in $P_{1} \cup P_{2} \cup P_{3}$.

Proof. If $C$ uses both $s^{\prime}$ and $t$, then we can partition $C$ into paths $Q_{1}, Q_{2}$ with endpoints $s^{\prime}$ and $t$. Since $\left|Q_{1}\right|+\left|Q_{2}\right|=2 \ell-2, Q_{1}^{\prime}=Q_{1} \cup s s^{\prime}$ and $Q_{2}^{\prime}=Q_{2} \cup s s^{\prime}$ are minimum $s t$-paths (i.e., $\left.Q_{1}^{\prime}, Q_{2}^{\prime} \in \overline{\mathcal{K}}\right)$. Let $F_{1}^{\prime}$ be the mate of $Q_{1}^{\prime}$ and $F_{2}^{\prime}$ be the mate of $Q_{2}^{\prime}$. Since $K_{1}$ and $K_{2}$ intersect $F_{1}^{\prime}$ and $F_{2}^{\prime}$ exactly once, $s s^{\prime} \in F_{1}^{\prime}$ and $s s^{\prime} \in F_{2}^{\prime}$. If $F_{1}, F_{2}, F_{3}$ are the mates of $P_{1}, P_{2}, P_{3}$, since $P_{1}, P_{2}, P_{3}$ are a 3 -path configuration, $s s^{\prime} \in F_{1}, s s^{\prime} \in F_{2}$, and $s s^{\prime} \in F_{3}$. Since $(\mathcal{F}, \mathcal{K})$ is a Lehman blocking pair, nonidentical elements of $\left\{P_{1}, P_{2}, P_{3}, Q_{1}^{\prime}, Q_{2}^{\prime}\right\}$ pairwise intersect in exactly $s s^{\prime}$. By Claim 4.6.2, nonidentical elements only share the vertices $\left\{s, s^{\prime}, t\right\}$. Since the vertices of $T-\left\{w, w^{\prime}\right\}$ are in $C$ and each $P_{i}$ has a vertex in $T-\left\{s, s^{\prime}, t\right\}$, we can assume that $Q_{1}^{\prime}=P_{2}$ and $Q_{2}^{\prime}=P_{3}$. It follows that the vertices of $T$ are in $P_{1} \cup P_{2} \cup P_{3}$.

Since $C$ does not use both $s^{\prime}$ and $t$ then either $s^{\prime} \notin T$ or $t \notin T$.

Claim 4.6.10. If $C$ does not use both $s^{\prime}$ and $t$, then there exists a minimum $T$-join $K \in$ $\mathcal{K}^{\prime}-\left\{K_{1}, K_{2}\right\}$.

Proof. By way of contradiction, suppose $K_{3} \ldots K_{r}$ are all st-paths. By Claim 4.6.2, they must be internally vertex disjoint. By Lehman Corollary 3.4.2, $E=K_{1} \cup \ldots \cup K_{r}$. Vertex $s^{\prime}$ must have degree three because 3 -path configuration $P_{1}, P_{2}, P_{3}$ uses $s^{\prime}$. Necessarily $K_{1}$ and $K_{2}$ use $s^{\prime}$ and so $s^{\prime} \in C$ and thus $t \notin C$. Each edge of $\delta(t)$ is on one of $K_{3} \ldots K_{r}$ and since $|\delta(t)| \geq 3$, this implies $r \geq 5$. If $v$ is a degree 2 vertex on one of $K_{3} \ldots K_{r}$, then $v \in T$ or else $M[\overline{\mathcal{K}}]$ is nonsingular, contradicting the fact $(\mathcal{F}, \mathcal{K})$ is a Lehman blocking pair. It follows that the vertices of $K_{3} \ldots K_{r}$ are in $C \cup\left\{w, w^{\prime}\right\}$. Since $C$ has $2 \ell-2$ vertices, it follows that $(r-2)(\ell-2) \leq 2 r$. Since $r \geq 5$, this implies $\ell=3$, a contradiction. Thus we may assume $K_{3} \in \overline{\mathcal{K}}$ is a minimum $T$-join.

Partition $C-\left(P_{1} \cup P_{2} \cup P_{3}\right)$ into a collection $\mathcal{S}$ of links. Vertices of $T$ are either on a link of $\mathcal{S}$ or in $P_{1} \cup P_{2} \cup P_{3}$. If $s^{\prime} \notin T$, no link has endpoint $s^{\prime}$. If $t \notin T$, no link has endpoint t. We say that link $S \in \mathcal{S}$ is in an $\mathcal{S}$-tripod if there exists a path $Y$ with endpoints $y, y^{\prime}$ such that 
1. internal vertices of $Y$ are not in $P_{1} \cup P_{2} \cup P_{3}$ or in any $S \in \mathcal{S}$,

2. $y$ is an internal vertex of $S$ that is in $T$, and

3. $y^{\prime}$ is not an endpoint of $S, y^{\prime} \neq s$ and $y^{\prime}$ is either a vertex of $P_{1}, P_{2}$ or $P_{3}$ or an internal vertex of link $S^{\prime} \in \mathcal{S}$ for $S^{\prime} \neq S$.

Claim 4.6.11. If $S \in \mathcal{S}$ is an odd link with both endpoints in $T$ then $S$ is in an $\mathcal{S}$-tripod.

Proof. Let $v, v^{\prime}$ be the endpoints of $S$ and $V_{s}=V(S) \cap T$. Since $S$ is odd, $\left|V_{s}\right|$ is odd. By Claim 4.6.1, $C^{\prime}=K_{1} \triangle K_{3}$ is a circuit. Thus $K_{3} \cap C^{\prime}$ is a collection $\mathcal{L}$ of pairwise vertex disjoint paths with endpoints in $T \cup\left\{w, w^{\prime}\right\}$ and internal vertices in $V-T-\left\{w, w^{\prime}\right\}$. Every vertex in $V_{s}$ is an endpoint of some path in $\mathcal{L}$. Let $\mathcal{L}^{+} \subseteq \mathcal{L}$ be the set of paths with exactly one endpoint in $V_{s}$. Since $\left|V_{s}\right|$ is odd, $\left|\mathcal{L}^{+}\right|$must be odd.

By way of contradiction, suppose all paths in $\mathcal{L}^{+}$have endpoints in $V_{s} \cup\left\{v, v^{\prime}\right\}$. Then $\mathcal{L}^{+}=\left\{L_{+}\right\}$; assume this path has endpoint $v$. Assume the edge of $S$ incident to $v$ is in $K_{1}$ and the edge of $S$ incident to $v^{\prime}$ is in $K_{2}$. Vertices of $V_{s} \cup v$ are incident to an edge of $K_{1} \cap S$ and to either an edge of a path in $\mathcal{L}-L_{+}$or an edge of $L_{+}$. The edges in $E^{\prime}=\left(K_{1} \cap S\right) \cup \mathcal{L}-L_{+}$is a disjoint union of circuits. However, $E^{\prime} \subseteq K_{1} \triangle K_{3}$ and since $C^{\prime}$ is a circuit containing all vertices in $T-\left\{w, w^{\prime}\right\}$, the vertices of $T-\left\{w, w^{\prime}\right\}$ are vertices of $S$. Since $v^{\prime} \in T-C$, either $v^{\prime}=w$ or $v^{\prime}=w^{\prime}$. So $v^{\prime}$ is on $P_{1}, v$ is on $P_{2}$, but there cannot be a $T$-vertex on $P_{3}$, a contradiction.

So there exists $L \in \mathcal{L}_{+}$with endpoint $y \in V_{s}$ and endpoint $y^{\prime}$ outside $V_{s} \cup\left\{v, v^{\prime}\right\}$. Let $y^{\prime}$ be first vertex of $L$ starting from $y$ which is a vertex of $P_{1}, P_{2}, P_{3}$ or some link of $\mathcal{S}-S$. The path $Y=L\left[y, y^{\prime}\right]$ is an $\mathcal{S}$-tripod since $y^{\prime} \neq s$ since $s \notin C^{\prime}$ by Claim 4.6.5.

Let $\left(G^{\prime}, T^{\prime}\right)$ and $\mathcal{S}^{\prime}$ be obtained from $(G, T)$ and $\mathcal{S}$ by performing the following operations.

1. For all $S \in \mathcal{S}$, contract all edges of $Q$ except for the two edges incident to the ends of $Q$.

2. Remove all even links from $\mathcal{S}$.

3. For any pair $S_{1}, S_{2} \in \mathcal{S}$ sharing endpoint $v$, contract edge of $S_{1}, S_{2}$ incident to $v$ and remove $S_{1}, S_{2}$ from $\mathcal{S}$.

4. If link $S \in \mathcal{S}$ has endpoint $v \in V\left(P_{i}\right)-\left\{s, s^{\prime}, t\right\}$ such that $v \notin T$ or there exists $v^{\prime} \in T-\left\{s^{\prime}, t, v\right\}$ in $V\left(P_{i}\right)$, then contract the edge of $S$ incident to $v$ and update $\mathcal{S}$ by removing $S$.

Since whenever $s^{\prime} \notin T$, no link has endpoint $s^{\prime}$, then $s^{\prime} \notin T^{\prime}$. Since whenever $t \notin T$, no link has endpoint $t$, then $t \notin T^{\prime}$. Moreover, since $s$ is not the endpoint of any link in $\mathcal{S}$, we maintain our 3-path configuration. 
Remark 4.6.12. $P_{1}, P_{2}, P_{3}$ is a 3-path configuration for $\left(G^{\prime}, T^{\prime}\right)$.

Claim 4.6.13. If not all vertices of $T^{\prime}$ are on $P_{1}, P_{2}, P_{3}$, then $\mathcal{S}^{\prime}$ contains a unique odd link $S^{\prime}$ such that for some internal vertex $y$

1. there exists paths $Q_{1}, Q_{2}, Q_{3}$ from $y$ to each of $v_{1}, v_{2}, v_{3}$ that uses no vertex of $P_{1} \cup$ $P_{2} \cup P_{3}$, or

2. there exists a path $Q$ from $v$ to $s^{\prime}$ or $t$.

Proof. This follows from two observations. Firstly, if more than two odd links remain, we can necessarily apply operation 3 or operation 4 . Secondly, if an odd link began in an $\mathcal{S}$ tripod, then it is in an $\mathcal{S}^{\prime}$ tripod throughout the procedure. Thus exactly one odd link remains and since it is an $\mathcal{S}^{\prime}$ tripod, one of the given cases must hold.

In the first case, deleting all edges except $P_{1} \cup P_{2} \cup P_{3}$ and contracting all remaining edges except $s s^{\prime}, \delta\left(v_{i}\right)$ for $i=1,2,3$ and $\delta(y)$ yields $\left(O_{5}, T_{5}\right)$. In the second case, deleting all edges except $P_{1} \cup P_{2} \cup P_{3} \cup Q$ and contracting all remaining edges except $s s^{\prime}$ and $\delta\left(v_{i}\right)$ for $i=1,2,3$ yields $\left(L_{1}, T_{1}\right)$ or $\left(L_{2}, T_{2}\right)$. This concludes the proof of correctness.

\subsubsection{Case 2: $\ell=3$}

Here the proof and algorithm are given simultaneously. Since $\ell=3$, and there exists minimum $T$-joins in $\overline{\mathcal{K}}$, it must be that $|T|=2,|T|=4$ or $|T|=6$.

Find $P_{1}, P_{2}, P_{3}$.

All the claims regarding finding 3-path configuration for $\ell \geq 4$ hold when $\ell=3$ except for Claim 4.6.6, In Claim 4.6.6, we required our st-paths to be length 4 to guarantee there was some edge such that neither of the endpoints were $s, s^{\prime}$ or $t$.

Claim 4.6.14. We can find a 3-path configuration.

Proof. As per proof in $\ell \geq 4$ section, we have paths $P_{i}=\left\{s s^{\prime}, s^{\prime} v_{i}, v_{i} t\right\}$ for $i=1,2,3$. If $s^{\prime} \notin T$, then necessarily $v_{i} \in T$ for all $i$ and we have a 3 -path configuration.

Otherwise, by way of contradiction, suppose $v_{1} \notin T$. By Claim 4.6.3, we can find minimum $T$-joins $K_{1}$ and $K_{2}$ intersecting in exactly edge $s^{\prime} v_{1}$. By Claim 4.6.1, $K_{1} \triangle K_{2}$ is a cycle $C$ and since $\ell=3,|C|=4$. Since $v_{1} \notin T$ and all vertices of $T-\left\{s^{\prime}\right\}$ are vertices 
of $C,|T|=4$. It follows that there exist $w_{1}, w_{2}, w_{3} \in T$ such that $K_{1}=\left\{v_{1} w_{1}, w_{2} w_{3}, v_{1} s^{\prime}\right\}$ and $K_{2}=\left\{w_{1} w_{2}, w_{3} v_{1}, v_{1} s^{\prime}\right\}$. If $s t$-T-cut $\delta(U)$ is the mate of minimum st-path $P_{1}$ then we may assume $s, v_{1} \notin U, s^{\prime}, t \in U$. Since $\delta(U)$ is not the mate of $K_{1}$ or $K_{2}, \delta(U) \cap K_{1}=$ $\delta(U) \cap K_{2}=\left\{s^{\prime} v_{1}\right\}$. It follows that $U \cap T=s^{\prime}$ and so $t \notin T$. Necessarily no minimum $T$-join contains edge $v_{1} t$ since $|T|=4$. This implies three minimum $T$-joins intersect in $v_{1} t$.

Since $v_{1}, t \notin T$, we can use edge $v_{1} t$ to find our 3 -path configuration. It remains to verify all minimum $T$-joins are disjoint from $\delta(t)$ so we can continue to use this property. Otherwise, some $T$-join uses two edges in $\delta(t)$ and this contradicts $\ell=3$ since it implies the existence of a length 2 st-path.

In summary, if choosing arbitrary $s s^{\prime} \in \delta(s)$ such that $s^{\prime} \in T$ and applying Lehman Corollary 3.4.6 does not lead to a 3-path configuration, then we can still find a 3-path configuration. Simply relabel $s$ and $t$ so that $s \notin T$, no minimum $T$-join intersects $\delta(s)$ and there exists edge $s s^{\prime} \in \delta(s)$ such that $s^{\prime} \notin T$; the above claim guarantees we can do this.

Note that since there exists a 3 -path configuration with $\left\{v_{1}, v_{2}, v_{3}\right\} \subseteq T,|T|=4$ or $|T|=6$. There are two cases: $s^{\prime} \notin T$ and $s^{\prime} \in T$.

Case 1: $s^{\prime} \notin T$

Consider edge $s^{\prime} v_{1}$. By Lehman Corollary 3.4.4, there exists a collection $\mathcal{K}^{\prime} \subseteq \overline{\mathcal{K}}=$ $\left\{K_{1}, K_{2} \ldots K_{r}\right\}$ intersecting in at most $s^{\prime} v_{1}$. Using Claim 4.6.3, we may assume $K_{1}, K_{2} \in \mathcal{K}$ intersect in exactly $s^{\prime} v_{1}$ and the mates of $K_{1}$ and $K_{2}$ also intersect in exactly $s^{\prime} v_{1}$. Since $s^{\prime} \notin T$, necessarily $|T|=4$. If $t \in T$, then we have found $\left(L_{1}, T_{1}\right)$. Otherwise, let $T=\left\{v_{1}, v_{2}, v_{3}, y\right\}$. Necessarily $K_{1}$ and $K_{2}$ are the union of

1. an edge joining two $T$ vertices, and

2. a length 2 path with internal vertex $s^{\prime}$ joining two $T$ vertices, one of which is $v_{1}$.

If the endpoint of the length 2 path in $K_{1}$ or $K_{2}$ is $y$, then we are done. Simply contract the edge joining $s^{\prime}$ and $y$, and delete all edges outside of $P_{1} \cup P_{2} \cup P_{3}$. Otherwise, the end of the length 2 path in $K_{1}$ is $v_{2}$ and the end of the length 2 path in $K_{2}$ is $v_{3}$. This implies there is an edge joining $v_{2}$ and $y$ and an edge joining $v_{3}$ and $y$.

In a similar way, there are minimum $T$-joins $K_{1}^{\prime}$ and $K_{2}^{\prime}$ intersecting in at most $s^{\prime} v_{2}$ and of the form above. If the endpoint of the length 2 path of $K_{1}$ or $K_{2}$ is $y$, then we are done. Otherwise, we are guaranteed there are edges $e_{1}, e_{2}, e_{3}$ joining $y$ to all of $\left\{v_{1}, v_{2}, v_{3}\right\}$. Deleting all edges except $P_{1} \cup P_{2} \cup P_{3} \cup\left\{e_{1}, e_{2}, e_{3}\right\}$ yields $\left(O_{5}, T_{5}\right)$. 
Case 2: $s^{\prime} \in T$

If $|T|=4$, then deleting all edges except $P_{1} \cup P_{2} \cup P_{3}$ yields $\left(L_{1}, T_{1}\right)$. Thus, we consider the case when $|T|=6$. Suppose $T=\left\{s, v_{1}, v_{2}, v_{3}, x, y\right\}$ where $x$ or $y$ is possibly $t$. If $x$ or $y$ is $t$, Claim 4.6.9 shows all $T$ vertices are on $P_{1} \cup P_{2} \cup P_{3}$. This is, however, impossible since $s \notin T$ and $\left|V\left(P_{1} \cup P_{2} \cup P_{3}\right)\right|=6$. By Lehman Corollary 3.4.4, there exists a collection $\mathcal{K}^{\prime} \subseteq \overline{\mathcal{K}}=\left\{K_{1}, K_{2} \ldots K_{r}\right\}$ intersecting in at most $s^{\prime} v_{1}$. Using Claim 4.6.3, we may assume $K_{1}, K_{2} \in \mathcal{K}$ intersect in exactly $s^{\prime} v_{1}$ and the mates of $K_{1}$ and $K_{2}$ also intersect in exactly $s^{\prime} v_{1}$. Since $|T|=6, s, v_{1} \in T, K_{1}=\left\{s v_{1}, e_{1}, e_{2}\right\}$ and $K_{2}=\left\{s v_{1}, e_{1}^{\prime}, e_{2}^{\prime}\right\}$, where $\left\{e_{1}, e_{2}\right\}$ and $\left\{e_{1}^{\prime}, e_{2}^{\prime}\right\}$ are matchings of $\left\{v_{2}, v_{3}, x, y\right\}$.

If any of $e_{1}, e_{2}, e_{1}^{\prime}, e_{2}^{\prime}$ join $x$ and $y$, then contracting the edge $x y$ and deleting all edges except $P_{1} \cup P_{2} \cup P_{3}$ yields $\left(L_{1}, T_{1}\right)$. Otherwise we may assume $e_{1}=v_{2} x, e_{2}=v_{3} y, e_{1}^{\prime}=v_{2} y$, $e_{2}^{\prime}=v_{3} x$. Contracting $\left\{e_{1}, e_{2}, e_{1}^{\prime}, e_{2}^{\prime}\right\}$, and deleting all edges except $P_{1} \cup P_{2} \cup P_{3}$ yields $\left(L_{1}, T_{1}\right)$.

\subsection{Remarks}

The problem of finding a fixed minor of a binary clutter is equivalent to the problem of finding a rooted minor in a binary matroid. It follows from recent developments in the matroid minor project that this problem can be solved in polynomial time [12]. Using these algorithms, it is possible to avoid using Lemma 4.3.3, Lemma 4.3.5 and Theorem 3.1.3 in the proofs of Theorem 4.1.1 and Theorem 4.1.2.

We have already introduced Corollary 1.3.4 of Theorem 4.1.1: given a graph and weight function on its edges, in polynomial time we can either find a maximum weight cut or find $K_{5}$ as an odd minor. Note that this result also follows from the graph minor testing algorithm with parity condition [21].

Proof of Corollary 1.3.4. Given $G$, let $\Sigma=E(G)$ and label some arbitrary vertex by $s=t$. If $\mathcal{F}$ is the clutter of odd-st-walks of $(G, \Sigma)$ then it is, in fact, the clutter of odd circuits of $G$. By Remark 4.3.1 and Proposition 4.3.2 we can, in polynomial time, find an extreme point $\bar{x}$ of $Q(\mathcal{F})$ that is optimal for $(2.5)$ together with a set of $n$ constraints of $Q(\mathcal{F})$ that define $\bar{x}$. If $\bar{x}$ is integer, then we may assume that it is the characteristic vector of a set of edges $B$ that intersects every odd circuit; i.e., $E(G)-B$ is the maximum cut. Otherwise, use Theorem 3.1.3 and Lemma 4.3.3 to find $I, J$ such that $\mathcal{F} / I \backslash J$ is isomorphic to $\mathcal{O}_{K_{5}}$. It can be then readily checked that $I$ forms a cut of $G$ and that $K_{5}$ is obtained by contracting all edges of $I$ and deleting parallel edges. 


\section{Chapter 5}

\section{Future Work, Related Problems and Conclusions}

\subsection{Future Work: Eulerian Multi-Commodity Flow Algorithms}

Recall that the fractional, integer and Eulerian integer flow problems are solved for graphs. That is, we know the minor minimal signed graphs such that $(G, \Sigma)$ does not admit an integer (or fractional) flow for every (or every Eulerian) weight function. Moreover, polynomial time algorithms for finding flows or obstructions are known for the fractional and integer cases. However, there does not exist a polynomial time algorithm corresponding to the result [13] on Eulerian integer multi-commodity flow.

Theorem 5.1.1 (Theorem 1.1.4). Suppose $(G, \Sigma)$ has no odd- $K_{5}$ minor. Then for every Eulerian weight function $w \in \mathbb{Z}_{+}^{E}$ satisfying the cut condition, $(G, \Sigma)$ has an integer $w$-flow.

We are interested in whether the construction in the above theorem can be used to obtain a polynomial time algorithm for the following problem: given $(G, \Sigma)$ and Eulerian $w \in \mathbb{Z}_{+}^{E}$, either show the cut condition is violated, show $(G, \Sigma)$ has an odd- $K_{5}$ minor, or find an integer $w$-flow.

We have no satisfactory answer to this question. Here we outline the unsuccessful strategy used and explain why doing the "obvious thing" did not work. In the positive direction, the construction in Theorem 1.1.4 leads to a finite algorithm for finding fractional flows; the general outline of this technique is found in Section 5.1.2. Additionally, 
we show that a certain type of incremental flow algorithm running in pseudo-polynomial time and finding either an obstruction or flow can be used to obtain a polynomial time algorithm to do the same. Although this seems like an unnatural construction, were the algorithm of Section 5.1.2 to run pseudo-polynomial time, it would result in exactly the incremental algorithm needed. For more details, see Section 5.1.3. We begin by discussing the transversal property of flows and outline two paradigms for designing flow algorithms: maintaining optimality and maintaining feasibility. The finite time algorithm of Section 5.1.2 maintains optimality while the capacity scaling algorithm of Section 5.1.3 maintains feasibility.

\subsubsection{Transversal Property, Underflows and Overflows}

Consider the unit weight case. An integer $\mathbb{1}$-flow is a set of edge disjoint odd circuits. A cover is a set of edges intersecting every odd circuit. Let $\mathcal{P}$ be a maximum set of edge disjoint odd circuits and let $B$ be a minimum cover. If $(G, \Sigma)$ has the Eulerian integer MFMC property, $|\mathcal{P}|=|B|$ and so $B$ is a transversal of $\mathcal{P}$. That is, each circuit in $\mathcal{P}$ contains exactly one edge in $B$ and each edge in $B$ is contained in exactly one circuit of $\mathcal{P}$. Given a maximum set of odd circuits $\mathcal{P}$, finding a minimum cover is equivalent to choosing an edge from each $P \in \mathcal{P}$ so that the chosen edges intersect every odd circuit of $(G, \Sigma)$. Symmetrically, given a minimum cover, finding an integer 1 -flow is equivalent to choosing a set of disjoint circuits - one through each edge in $B$.

In the weighted case, there is an similar transversal property. Let $B$ be some minimum cover of $(G, \Sigma)$ for Eulerian weight function $w$. If $(G, \Sigma)$ has the Eulerian integer MFMC property, then any maximum integer $w$-flow $y \in \mathbb{Z}_{+}^{\mathcal{C}_{1}}$ is such that $\sum\left(y_{C}: d \in C \in \mathcal{C}_{1}\right)=w_{d}$ for all $d \in B$. That is, the minimum cover is (in a weighted sense) a transversal of the maximum $w$-flow. Suppose we are given minimum cover $B$. Finding a $w$-flow can be viewed as choosing, for each $d \in B, w_{d}$ circuits intersecting $B$ in exactly $\{d\}$.

Algorithm design paradigms for flow problems fall into two categories: algorithms that maintain optimality and algorithms that maintain feasibility. For example, Ford Fulkerson [11] maintains feasibility while Preflow Push [15] maintains optimality. This dichotomy follows through for multi-commodity flow instances.

We call $y \in \mathbb{Z}_{+}^{\mathcal{C}_{1}}$ an overflow if for each $d \in \Sigma, \sum\left(y_{C}: d \in C \in \mathcal{C}\right)=w_{d}$; i.e., if it satisfies (1.1). An overflow satisfies the demand constraints but not necessarily the capacity constraints. If $\sum\left(y_{C}: e \in C \in \mathcal{C}\right)=w_{e}+\Gamma_{e}$ for $\Gamma_{e} \geq 1, \Gamma_{e} \in \mathbb{Z}$, we call $e$ an inflated edge. The total inflation of overflow $y \in \mathbb{Z}_{+}^{\mathcal{C}_{1}}$ is $\Gamma(y)=\sum\left(\Gamma_{e}: e\right.$ is inflated edge). An algorithm maintaining feasibility begins with overflow $y$ having total inflation $\Gamma(y)$ and 
tries to construct overflow $y^{\prime}$ having total inflation $\Gamma\left(y^{\prime}\right)$ such that $\Gamma\left(y^{\prime}\right)<\Gamma(y)$. Overflows are used in Section 5.1.2 to describe the finite time constructive algorithm arising from Theorem 1.1.4.

We call $y \in \mathbb{Z}_{+}^{\mathcal{C}_{1}}$ an underflow if for each $e \in E, \sum\left(y_{C}: e \in C \in \mathcal{C}_{1}\right) \leq w_{e}$; i.e, if it satisfies (1.2). An underflow satisfies the capacity constraints but not necessarily the demand constraints. An algorithm maintaining feasibility works to construct from underflow $y$, an underflow $y^{\prime}$ such that $\sum\left(y_{C}: C \in \mathcal{C}\right)<\sum\left(y_{C}^{\prime}: C \in \mathcal{C}\right)$. Since the value of the underflow increases by at least 1 by doing this, eventually a flow will be found. The deficiency of underflow $y$ is defined as the difference between the size of the minimum cover and the amount of satisfied demand. The deficiency of underflow $y$ is given by $\Delta(y)=w(\Sigma)-\sum\left(y_{C}: C \in \mathcal{C}_{1}\right)$. Underflows are used in Section 5.1.3 to show that the existence of an incremental pseudo-polynomial time algorithm finding integer flows or obstructions implies the existence of a polynomial time algorithm doing the same.

In summary, we have three types of flows: underflows satisfy capacity constraints; overflows satisfy demand constraints; flows satisfy demand and capacity constraints.

\subsubsection{Finite Time Algorithm Arising from Theorem 1.1.4}

Suppose we are given $(G, \Sigma)$ and Eulerian $w \in \mathbb{Z}_{+}^{E}$. The first step is to find a minimum weight signature $\Sigma_{w}$. Note that we can either find such a signature or find an odd- $K_{5}$ minor in polynomial time because of Theorem 4.1.1. If we find an odd- $K_{5}$ minor, we are done. If $w\left(\Sigma_{w}\right)<w(\Sigma)$, then the cut condition is violated and we are done. Otherwise, we conclude that $\Sigma$ is indeed a minimum weight signature and thus the cut condition holds. Since the cut condition holds, finding an integer $w$-flow is equivalent to finding an integer packing of odd circuits of $(G, \Sigma)$ of size $w(\Sigma)$.

Note although that flow decomposition techniques admit polynomial size representations for overflows, for the purposes of discussion we will simply work with overflows represented by $y \in \mathbb{Z}_{+}^{\mathcal{C}_{1}}$.

Generate an overflow by choosing for each $d \in \Sigma$ exactly $w_{d}$ circuits intersecting $\Sigma$ in exactly $\{d\}$. If there is no inflated edge, then we already have a flow. Otherwise, there exists an edge $e \in E$ such that $\sum\left(y_{C}: e \in C \in \mathcal{C}\right)=w_{e}+\Gamma_{e}$ for $\Gamma_{e} \geq 2$. The goal is to decrease $\Gamma_{e}$ by 2 .

First, find circuits $C_{1}, C_{2}, C_{3}$ using edge $e$ with $y_{C_{i}} \geq 0$. We update circuits $C_{1}, C_{2}, C_{3}$ until none are removable. Consider weight function $w^{\prime}$ with $w_{e}^{\prime}=w_{e}-1$ for all $e \in C_{i}$ and

$w_{e}^{\prime}=w_{e}$ for all $e \notin C_{i}$. Circuit $C_{i}$ is removable if the weight of the minimum cover of 
$G$ with weight function $w$ is exactly one more than the weight of a minimum cover of $G$ with weight function $w^{\prime}$. If $C_{i}$ is removable, we update our weight function to $w^{\prime}$ and 'set $C_{i}$ aside'; applying this reduction until none of $C_{1}, C_{2}, C_{3}$ are removable suppose we set aside $k(C)$ copies of $C \in \mathcal{C}$. If we terminate in this iteration, we increase $y_{C}$ by $k(C)$ for all $C \in \mathcal{C}$ to obtain a solution. If we do not terminate in this iteration, we increase $y_{C}$ by $k(C)$ for all $C \in \mathcal{C}$ to obtain the overflow for the next iteration. After this step, we have some new weight function $w^{\prime}$ and circuits $C_{1}, C_{2}, C_{3}$ using $e$, none of which are removable.

If $w_{e}^{\prime} \geq 2$, using the construction in [13], either:

1. Untangling step: Obtain circuits $C_{1}^{\prime}, C_{2}^{\prime}, C_{3}^{\prime}$ such that $E\left(C_{1}^{\prime} \cup C_{2}^{\prime} \cup C_{3}^{\prime}\right) \subseteq E\left(C_{1} \cup C_{2} \cup\right.$ $C_{3}$ ) (counting multiplicities) and $e \notin C_{1}, e \notin C_{2}$.

2. Find odd $-K_{5}$ minor.

In the second case, we are finished. In the first case set $y_{C_{i}}=y_{C_{i}}-1$ and $y_{C_{i}^{\prime}}=y_{C_{i}^{\prime}}+1$ and continue by choosing some other inflated edge $w_{e}$.

If $w_{e}^{\prime} \leq 1$, we have to apply the algorithm recursively on $(G-e, \Sigma-e)$ to do the untangling step. Note that if $\Gamma_{e}=1$ for some $e$, we need to untangle in a different way; the ability to do so follows from the fact the original weight function was Eulerian.

Although this algorithm will eventually terminate since we call recursively on increasingly smaller graphs, the recursive calls lead to an exponential running time. The use of removable circuits is not ideal; the main issue is that we have no relationship of the type 'If a circuit is removable for weight function $w_{1}$, then it is also removable for related weight function $w_{2}$ '. If we could guarantee a removable circuit for the original weight function could be located every polynomially many steps, this would in fact imply a pseudo-polynomial time algorithm for the problem.

\subsubsection{Capacity Scaling}

Suppose we have an algorithm with the following input and output.

- Input:

1. $(G, \Sigma)$, and

2. $w \in \mathbb{Z}_{+}^{E}$ such that the cut condition holds, and

3. underflow $y$ with deficiency $\Delta(y)$

- Output either

1. $w$-flow $y^{\prime}$, or

2. $I, J \subseteq E(G)$ such that $(G, \Sigma) / I \backslash J$ is odd- $K_{5}$ 
We will call such an algorithm a packAdditional algorithm.

The algorithm discussed in Section 5.1.2 is, in fact, a packAdditional algorithm. Underflow $y$ provides a mechanism for choosing our starting set of circuits for the algorithm; if $\sum\left(y_{C}: d \in C \in \mathcal{C}_{1}\right)=z_{d}$, then we choose an additional $w_{d}-z_{d}$ circuits intersecting $\Sigma$ in exactly $\{d\}$. This algorithm does not run in pseudo-polynomial time in deficiency $\Delta(y)$; however, this had been the initial hope.

A packAdditional algorithm running in pseudo-polynomial time would in fact imply a polynomial time algorithm for the problem of finding a $w$-flow or odd- $K_{5}$ for multicommodity flow instance for which the cut condition holds. This is proven below using a capacity scaling scheme. Note that this scheme requires the ability to calculate minimum covers and thus could not be applied for arbitrary multi-commodity flow instances. Since multi-commodity flow is strongly NP-hard for $|\Sigma| \geq 2$, schemes of this sort would be uninteresting since a pseudo-polynomial time algorithm does not exist unless $P=N P$. Additionally, devising packAdditional type algorithms is difficult since it requires a strong understanding as to what types of partial solutions can lead to complete solutions.

The capacity scaling scheme used here is similar to the one used for Ford Fulkerson (see [11]). The main idea is to use the large units of available capacity first and then add bits of accuracy in further iterations. If the cut condition holds for initial weight function $w$, it may not hold for $w^{\prime}$ obtained by restricting $w$ to the highest order bit of any entry. Accordingly, we must adjust our demand at each step to guarantee we are still working with an instance satisfying the cut condition. Also note that we must decrease demand on all demand edges since we do not know exactly how to redistribute demand to restore the cut condition otherwise. The key observation that makes the scheme work is that if we choose weights at one iteration such that the cut condition holds, the violation in the next iteration is bounded by some function of $|E|$ and $|\Sigma|$.

In stating the capacity scaling scheme, packAdditional $(G, \Sigma, w, y)$ denotes a call to the packAdditional algorithm for signed graph $(G, \Sigma)$, weight function $w$, and underflow $y$. Let $W$ denote the largest entry of $w$. The capacity scaling algorithm is as follows.

Let $k=\left\lfloor\log _{2} W\right\rfloor$.

Let $y=0$.

While $k>0$ Do

Let $w^{\prime}=\left\lfloor w / 2^{k}\right\rfloor$.

Find $\tau_{w^{\prime}}$, the weight of minimum signature of $(G, \Sigma)$ for weight function $w^{\prime}$.

Let the excess $E$ be $\sum\left(w_{d}^{\prime}: d \in \Sigma\right)-\tau_{w}^{\prime}$.

Set $w_{d}^{\prime \prime}=\max \left(w_{d}^{\prime}-E, w_{d}(2 y)\right)$.

$y=\operatorname{packAdditional}\left(G, \Sigma, w^{\prime \prime}, 2 y\right)$. 
End

To analyze the running time of this algorithm, first note that we need to find $\tau_{w^{\prime}}$. Theorem 4.1.1 of this thesis shows we can efficiently find $\tau_{w^{\prime}}$ or show odd- $K_{5}$ is a minor of $(G, \Sigma)$. The outer loop is performed $\log _{2} W$ times. As long as the deficiency for each packAdditional call is polynomial in $|\Sigma|$ and $|E|$, this would imply that the entire algorithm runs in polynomial time. The below lemma shows that the deficiency in each iteration is polynomially bounded.

Lemma 5.1.2. Let $w$ be the weight function in iteration $i$ of the outer loop and $w^{\prime}$ be the weight function in iteration $i+1$ of the outer loop. Then $\sum\left(w_{d}^{\prime}: d \in \Sigma\right) \leq 2 \sum\left(w_{d}: d \in\right.$ $\Sigma)+|\Sigma||E|^{2}$.

Proof. If we assume $w^{\prime}$ satisfies the cut condition (as it would in the last iteration), the analysis is straightforward. If $\Sigma_{w}$ is a minimum weight signature of $(G, \Sigma)$ for weight function $w$, then $\Sigma_{w}=\Sigma \triangle \delta(S)$ for $S \subseteq V$. Since $w^{\prime}\left(\Sigma_{w}\right)=w^{\prime}(\Sigma \triangle \delta(S)) \geq w^{\prime}(\Sigma)$ and $w_{e}^{\prime}=2 w_{e}+r_{e}$ for $r_{e} \in\{0,1\}, w(\Sigma)-2 w\left(\Sigma_{w}\right) \leq|E|$. In other words, $2 \tau_{w} \geq \tau_{w^{\prime}}-|E|$ and the excess in each iteration is bounded by $|E|$. Accordingly, the difference in demand between two iterations in bounded by $|E||\Sigma|$ since we subtract the excess from every demand edge. Lastly, we note that if $w^{\prime}$ does not satisfy the cut condition, the above analysis holds by comparing $\Sigma_{w}$ and $\Sigma_{w^{\prime}}$ instead.

\subsection{Other Related Problems}

The Flowing and Cycling Conjectures (Conjectures 1.2.4 and 1.2.3) remain open for many classes of clutters. In Section 2.4.4 and 2.4.5, we showed that for duals of even cut and even cycle matroids, the single commodity Cycling Conjecture implies the Four Colour Theorem. This suggests the conjecture may be difficult to resolve even for the single commodity case.

\subsubsection{Cuts with Parity Conditions}

The separation problem for st-T-cuts has some generalizations of interest. As mentioned in Section 4.4, one generalization is finding $T_{1}$-odd, $T_{2}$-odd cuts. That is, given $G=(V, E)$ and $T_{1}, T_{2} \subseteq V$ with $\left|T_{1}\right|,\left|T_{2}\right|$ even, we want to find a minimum weight cut $\delta(S)$ such that $\left|T_{1} \cap S\right|$ is odd and $\left|T_{2} \cap S\right|$ is odd. An uncrossing lemma of [16] shows that minimum weight $T_{1}$-odd, $T_{2}$-odd cuts can be found efficiently. Faster algorithms for this problem have been developed; see [14] and [2]. One related question is whether we can get efficient 
algorithms for different parity conditions. A $T_{1}$-odd, $T_{2}$-even cut is $\delta(S)$ for $S \subseteq V$ such that $\left|T_{1} \cap S\right|$ is odd and $\left|T_{2} \cap S\right|$ is even. Using an uncrossing lemma, we can again find minimum $T_{1}$-odd, $T_{2}$-even cuts efficiently $\left(\delta(S)\right.$ is $T_{1}$-odd and $T_{2}$-even if and only if it is $T_{1}$-odd and $T_{1} \triangle T_{2}$-odd). A $T_{1}$-even, $T_{2}$-even cut is $\delta(S)$ for $S \subseteq V$ such that $\left|T_{1} \cap S\right|$ is even and $\left|T_{2} \cap S\right|$ is even. The uncrossing lemma does not follow in this case; in fact, it is an open problem to devise an efficient algorithm finding minimum $T_{1}$-even, $T_{2}$-even cuts.

\subsubsection{Minimally Imperfect Graphs}

A matrix 0,1 matrix $M$ with no column of zeroes is perfect if the set packing polytope $\left\{x \in \mathbb{R}_{+}^{n}: M x \leq \mathbb{1}\right\}$ is integral. One can show that a matrix is perfect if and only if it is the clique-node matrix of a perfect graph. Taking induced subgraphs corresponds to taking column submatrices and we define matrix $M$ to be minimally imperfect if the corresponding set packing polytope is not integral but the set packing polytope for every column submatrix is integral. The minimally imperfect graphs are known to be the odd holes and odd antiholes. There exist polynomial time algorithms to recognize if a graph has an odd hole or odd antihole and thus check if $M$ is perfect. For more background information about this problem see, [6] Chapter 3.2 and Chapter 3.3.

A result akin to Lehman's Theorem for minimally non ideal clutters holds for minimally imperfect graphs. Bruce Shepherd [31] asked if an analogue to the constructive version of the Lehman's Theorem (Theorem 3.1.3) holds for minimally imperfect graphs. This could lead to alternative algorithms for finding odd holes or antiholes. Since the corresponding separation problem requires checking for given $k$ if there is a clique of size at least $k$ and thus is hard to solve, no analogous result to Theorem 3.1.3 is possible.

\subsection{Conclusions}

In conclusion, we used a constructive version of Lehman's Theorem to show that for lifts of graphic and cographic matroids there exist polynomial time algorithms finding either a fractional flow and cover of the same value, a cut condition violation, or an obstruction. Our attempts to get analogous results for Eulerian integer multi-commodity flows were foiled, leaving this as an open problem. 


\section{References}

[1] A. Abdi and B. Guenin. Personal communication, 2012.

[2] A. Benczúr and O. Fülöp. Fast algorithms for even/odd minimum cuts and generalizations. In Algorithms - ESA 2000, volume 1879 of Lecture Notes in Computer Science, pages 88-99. Springer Berlin Heidelberg, 2000.

[3] R.E. Bixby. l-matrices and a characterization of binary matroids. Discrete Mathematics, 8:139- 145, 1974.

[4] W.G. Bridges and H.J. Ryser. Combinatorial designs and related systems. Journal of Algebra, 13:432- 446, 1969.

[5] M. Conforti and E.J. Johnson. Two min-max theorems for graphs noncontractible to a four wheel (technical report). IBM Thomas J. Watson Research Center, Yorktown Heights, New York, 1987.

[6] G. Cornuéjols. Combinatorial Optimization: Packing and Covering, volume 72. SIAM, Philadelphia, 2001.

[7] G. Cornuejols and B. Guenin. Ideal binary clutters, connectivity, and a conjecture of seymour. SIAM Journal of Discrete Mathematics, 15(3):329-352, 2002.

[8] N.G. de Bruijn and P. Erdös. On a combinatorial problem. Proc. Kon. Ned. Akad. v. Wetensch, 51:1277-1279.

[9] R.J. Duffin. The extremal length of a network. Journal of Mathematical Analysis and Applications, 5(2):200 - 215, 1962.

[10] S. Even, A. Itai, and A. Shamir. On the complexity of time table and multi-commodity flow problems. Symposium on the Foundations of Computer Science, 16:184-193, 1975. 
[11] L.R. Ford and D.R. Fulkerson. Maximal flow through a network. Canadian Journal of Mathemtics, 8:399 - 404, 1956.

[12] J.F. Geelen, A.M.H. Gerards, and G. Whittle. Towards a matroid-minor structure theory. Combinatorics, Complexity, and Chance. A tribute to Dominic Welsh, pages $72-82,2007$.

[13] J.F. Geelen and B. Guenin. Packing odd-circuits in eulerian graphs. Journal of Combinatorial Theory, Series B, 86(2):280 - 295, 2002.

[14] M.X. Goemans and V.S. Ramakrishnan. Minimizing submodular functions over families of sets. Combinatorica, 15:499-513, 1995.

[15] Andrew V. Goldberg and Robert E. Tarjan. A new approach to the maximum-flow problem. J. ACM, 35(4):921-940, October 1988.

[16] M. Grötschel, L. Lovász, and A. Schrijver. Geometric Algorithms and Combinatorial Optimization. Springer Verlag, 1988.

[17] M. Grötschel and W.R. Pulleyblank. Weakly bipartite graphs and the max-cut problem. Operations Research Letters, 1(1):23 - 27, 1981.

[18] B. Guenin. A characterization of weakly bipartite graphs. Journal of Combinatorial Theory, Series B, 83(1):112 - 168, 2001.

[19] Bertrand Guenin. Integral polyhedra related to even-cycle and even-cut matroids. Mathematics of Operations Research, 27(4):693-710, 2002.

[20] Bertrand Guenin. A short proof of Seymour's characterization of the matroids with the max-flow min-cut property. Journal of Combinatorial Theory, Series B, 86(2):273 $-279,2002$.

[21] K. Kawarabayashi, B. Reed, and P. Wollan. The graph minor algorithm with parity conditions. In Foundations of Computer Science (FOCS), 2011 IEEE 52nd Annual Symposium on, pages 27 -36, Oct. 2011.

[22] A. Lehman. On the width-length inequality. Mathematical Programming, 17:403-417, 1979 .

[23] A. Lehman. On the width-length inequality and degenerate projective planes. In W. Cook and P.D. Seymour, editors, Polyhedral Combinatorics, DIMACS Series in Discrete Mathematics and Theoretical Computer Science 1, pages 101-105. American Mathematical Society, Providence, RI, 1990. 
[24] J. Oxley. Matroid theory, volume 21 of Oxford Graduate Texts in Mathematics. Oxford University Press, Oxford, second edition, 2011.

[25] I. Pivotto. Even-cycle and even-cut matroids. Ph.D thesis, University of Waterloo, 2011.

[26] N. Robertson, D. Sanders, P.D. Seymour, and R. Thomas. The four colour theorem. Journal of Combinatorial Theory, Series B, 70:2-44, 1997.

[27] N. Robertson, P.D. Seymour, and R. Thomas. Tutte's edge-coloring conjecture. Journal of Combinatorial Theory, Series B, 70:166 - 183, 1997.

[28] A. Schrijver. A short proof of Guenin's characterization of weakly bipartite graphs. Journal of Combinatorial Theory, Series B, 85:255 - 260, 2002.

[29] P.D. Seymour. The matroids with the max-flow min-cut property. Journal of Combinatorial Theory, Series B, 23(23):189 - 222, 1977.

[30] P.D. Seymour. Matroids and multicommodity flows. European Journal of Combinatorial Theory, Series 2, 28:257 - 290, 1981.

[31] B. Shepherd. Personal communication, 2012.

[32] P.G. Tait. Note on a theorem in geometry of position. Transactions of the Royal Society of Edinburgh, 29:657-660, 1880.

[33] K. Truemper. Max-flow min-cut matroids: Polynomial testing and polynomial algorithms for maximum flow and shortest routes. Mathematics of Operations Research, 12(1):pp. 72-96, 1987.

[34] W.T. Tutte. On the algebraic theory of graph colorings. Journal of Combinatorial Theory, 1:15 - 50, 1966.

[35] T. Zaslavsky. Biased graphs. i. bias, balance, and gains. Journal of Combinatorial Theory, Series B, 47:32 - 52, 1989. 